\title{
Uniform convergence to equilibrium for a family of drift-diffusion models with trap-assisted recombination and the limiting Shockley-Read-Hall model
}

\author{
Klemens Fellner ${ }^{1} \cdot$ Michael Kniely $^{2}$ (])
}

Received: 23 July 2019 / Accepted: 11 April 2020 / Published online: 7 May 2020

(C) The Author(s) 2020

\begin{abstract}
In this paper, we establish convergence to equilibrium for a drift-diffusion-recombination system modelling the charge transport within certain semiconductor devices. More precisely, we consider a two-level system for electrons and holes which is augmented by an intermediate energy level for electrons in so-called trapped states. The recombination dynamics use the mass action principle by taking into account this additional trap level. The main part of the paper is concerned with the derivation of an entropy-entropy production inequality, which entails exponential convergence to the equilibrium via the so-called entropy method. The novelty of our approach lies in the fact that the entropy method is applied uniformly in a fast-reaction parameter which governs the lifetime of electrons on the trap level. Thus, the resulting decay estimate for the densities of electrons and holes extends to the corresponding quasisteady-state approximation.
\end{abstract}

Keywords Drift-diffusion-recombination models $\cdot$ Semiconductors $\cdot$ ShockleyRead-Hall · Trapped states · Entropy method · Convergence to equilibrium ·

Exponential rate of convergence $\cdot$ Quasi-steady-state approximation · Fast-reaction limit

Mathematics Subject Classification Primary 35K57 - Secondary 35B40 · 35B45 · 82D37

Michael Kniely

michael.kniely@ist.ac.at

Klemens Fellner

klemens.fellner@uni-graz.at

1 Institute of Mathematics and Scientific Computing, University of Graz, Heinrichstraße 36, 8010 Graz, Austria

2 Institute of Science and Technology Austria (IST Austria), Am Campus 1, 3400 Klosterneuburg, Austria 


\section{Introduction and main results}

The formulation and mathematical analysis of drift-diffusion type semiconductor models reach back to the middle of the last century, see e.g. [18, 20, 24] and the references therein; yet these models still form a highly relevant workhorse in the simulation of semiconductor devices and batteries.

Physically, drift-diffusion models describe the transport of charge carriers via diffusion and convection governed by electric fields. In semiconductors, charge carriers are electrons and holes (positively charged quasi-particles, which represent the absence of an electron). Pairs of electrons and holes can be "generated" and "destroyed" by recombination processes. Generation of an electron-hole pair occurs when an electron is lifted from a low-energy valence band to a high-energy conduction band, where electrons are mobile-leaving behind an equally mobile hole in the valence band. A pivotal generation-recombination model was formulated by Shockley, Read and Hall [16, 21]. Mathematically, Shockley-Read-Hall recombination introduces quadratic non-linear reaction terms into the drift-diffusion dynamics.

A derivation of the Shockley-Read-Hall model considers a generation-recombination process as sketched in Fig. 1. It assumes that appropriately distributed foreign atoms in the crystal lattice of the semiconductor material facilitate the generation of electron-hole pairs by providing in-between energy levels, requiring smaller amounts of energy for each step. Since electrons are immobile at these in-between energy levels, they are called trapped states. Also their maximal density is limited. The Shockley-Read-Hall model of electron-hole recombination is obtained as a quasi-steady-state approximation of the trapped-state dynamics as detailed in the following.

We denote the charge densities of electrons, holes and trapped states by $n, p$ and $n_{t r}$ and consider the following PDE-ODE drift-diffusion-recombination system:

$$
\left\{\begin{aligned}
\partial_{t} n & =\nabla \cdot J_{n}(n)+R_{n}\left(n, n_{t r}\right), \\
\partial_{t} p & =\nabla \cdot J_{p}(p)+R_{p}\left(p, n_{t r}\right), \\
\varepsilon \partial_{t} n_{t r} & =R_{p}\left(p, n_{t r}\right)-R_{n}\left(n, n_{t r}\right),
\end{aligned}\right.
$$

with the drift-diffusion fluxes and reaction terms

Fig. 1 A schematic picture illustrating the allowed transitions of electrons between the various energy levels

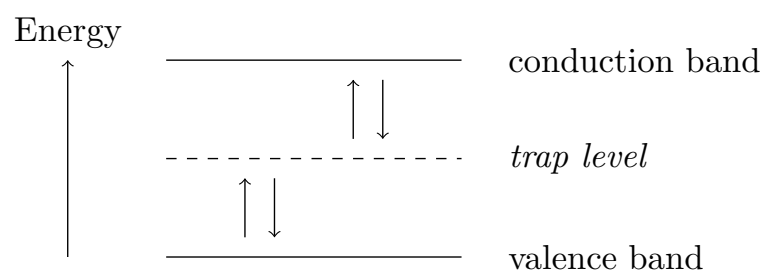




$$
\begin{aligned}
& J_{n}:=\nabla n+n \nabla V_{n}=\mu_{n} \nabla\left(\frac{n}{\mu_{n}}\right), \quad \mu_{n}:=e^{-V_{n}}, \\
& J_{p}:=\nabla p+p \nabla V_{p}=\mu_{p} \nabla\left(\frac{p}{\mu_{p}}\right), \quad \mu_{p}:=e^{-V_{p}}, \\
& R_{n}:=\frac{1}{\tau_{n}}\left(n_{t r}-\frac{n}{n_{0} \mu_{n}}\left(1-n_{t r}\right)\right), \\
& R_{p}:=\frac{1}{\tau_{p}}\left(1-n_{t r}-\frac{p}{p_{0} \mu_{p}} n_{t r}\right) .
\end{aligned}
$$

The constants $n_{0}, p_{0}, \tau_{n}, \tau_{p}>0$ are positive recombination parameters and $\varepsilon \in\left(0, \varepsilon_{0}\right]$ for arbitrary $\varepsilon_{0}>0$ is a positive relaxation parameter. $V_{n}$ and $V_{p}$ represent external time-independent potentials.

The reaction term $R_{n}$ models transitions of electrons from the trap level to the conduction band (proportional to $n_{t r}$ ) and vice versa (proportional to $-n\left(1-n_{t r}\right)$ ), where the maximum capacity of the trap level is normalised to one. The analogue processes with respect to the valence band are described by $R_{p}$. Note that the rate of hole generation is equivalent to the rate of electrons moving from the valence band to the trap level, which is proportional to $\left(1-n_{t r}\right)$. Similarly, the annihilation of a hole corresponds to an electron that jumps from the trap level to the valence band, which yields a reaction rate proportional to $-p n_{t r}$. Moreover, $n_{0}, p_{0}>0$ represent reference levels for the charge concentrations $n$ and $p$, while $\tau_{n}, \tau_{p}>0$ are inverse reaction parameter. Note that the concentration of trapped states satisfies $n_{t r} \in[0,1]$ provided this holds true for their initial concentration (cf. Theorem 1.1).

The dynamical equation for $n_{t r}$ in (1) is an ODE in time and pointwise in space with a right hand side depending on $n, p$ and $n_{t r}$ via $R_{n}$ and $R_{p}$. We stress that there is no drift-diffusion term for $n_{t r}$ since trapped electrons are immobile. This is due to the correlation between foreign atoms and the corresponding trap levels which are locally bound near these crystal impurities.

The parameter $\varepsilon>0$ models the lifetime of trapped states, where lifetime refers to the expected time until an electron in a trapped state moves either to the valence or the conduction band. The Shockley-Read-Hall recombination model is obtained in the (formal) limit $\varepsilon \rightarrow 0$, where the concentration of trapped states is determined from the algebraic relation $0=R_{p}\left(p, n_{t r}\right)-R_{n}\left(n, n_{t r}\right)$ :

$$
n_{t r}^{q s s a}=\frac{\tau_{n}+\tau_{p} \frac{n}{n_{0} \mu_{n}}}{\tau_{n}+\tau_{p}+\tau_{n} \frac{p}{p_{0} \mu_{p}}+\tau_{p} \frac{n}{n_{0} \mu_{n}}} .
$$

In this quasi-steady-state approximation, the density of trapped states $n_{t r}$ and its evolution are eliminated from system (1), while the evolutions of the charge carriers $n$ and $p$ are subject to the Shockley-Read-Hall recombination terms 


$$
R_{n}\left(n, n_{t r}^{q s s a}\right)=R_{p}\left(p, n_{t r}^{q s s a}\right)=\frac{1-\frac{n p}{n_{0} p_{0} \mu_{n} \mu_{p}}}{\tau_{n}\left(1+\frac{p}{p_{0} \mu_{p}}\right)+\tau_{p}\left(1+\frac{n}{n_{0} \mu_{n}}\right)} .
$$

A rigorous proof of this quasi-steady-state approximation has been performed in [15], even for more general models. See also [18] for semiconductor models with reaction terms of Shockley-Read-Hall-type.

We complete the mathematical description by considering system (1) on a bounded domain $\Omega \subset \mathbb{R}^{m}, m \geq 1$, with sufficiently smooth boundary $\partial \Omega$. Without loss of generality, we suppose that the volume of $\Omega$ is normalised, i.e. $|\Omega|=1$, which can be achieved by an appropriate scaling of the spatial variables.

We impose no-flux boundary conditions for $J_{n}$ and $J_{p}$,

$$
\hat{n} \cdot J_{n}=\hat{n} \cdot J_{p}=0 \quad \text { on } \partial \Omega,
$$

where $\hat{n}$ denotes the outer unit normal vector on $\partial \Omega$, and we prescribe non-negative and bounded initial data $n_{I}, p_{I}, n_{t r, I} \in L^{\infty}(\Omega)$ together with $\left\|n_{t r, I}\right\|_{L^{\infty}(\Omega)} \leq 1$. As a consequence, the following charge conservation law holds:

$$
\int_{\Omega}\left(n-p+\varepsilon n_{t r}\right) d x=\int_{\Omega}\left(n_{I}-p_{I}+\varepsilon n_{t r, I}\right) d x=: M
$$

with $M \in \mathbb{R}$. Finally, the potentials $V_{n}$ and $V_{p}$ are assumed to satisfy

$$
V_{n}, V_{p} \in W^{2, \infty}(\Omega) \quad \text { and } \quad \hat{n} \cdot \nabla V_{n}, \hat{n} \cdot \nabla V_{p} \geq 0 \quad \text { on } \partial \Omega,
$$

where the last condition means that the potentials are confining.

The main goal of this paper is to prove exponential convergence to equilibrium of system (1)-(4) with explicit bounds on rates and constants, which are independent of the relaxation time $\varepsilon$. We therefore consider $\varepsilon \in\left(0, \varepsilon_{0}\right]$ for arbitrary but fixed $\varepsilon_{0}>0$. Our study also includes the limiting case $\varepsilon=0$.

The main tool in quantifying the large-time behaviour of global solutions to system (1) is the entropy functional

$$
\begin{aligned}
E\left(n, p, n_{t r}\right)=\int_{\Omega} & \left(n \ln \frac{n}{n_{0} \mu_{n}}-\left(n-n_{0} \mu_{n}\right)+p \ln \frac{p}{p_{0} \mu_{p}}-\left(p-p_{0} \mu_{p}\right)\right. \\
& \left.+\varepsilon \int_{1 / 2}^{n_{t r}} \ln \left(\frac{s}{1-s}\right) d s\right) d x .
\end{aligned}
$$

For $n$ and $p$, we encounter contributions of the Boltzmann-entropy form $a \ln a-(a-1) \geq 0$, whereas $n_{t r}$ enters the entropy functional via a non-negative integral term. Note that the integral $\int_{1 / 2}^{n_{t r}} \ln \left(\frac{s}{1-s}\right) d s$ is non-negative and well-defined for all $n_{t r}(x) \in[0,1]$. By introducing the entropy production functional

$$
P:=-\frac{d}{d t} E
$$


it holds (formally) true along solution trajectories of system (1), (2) that

$$
\begin{aligned}
P\left(n, p, n_{t r}\right)= & \int_{\Omega}\left(\frac{\left|J_{n}\right|^{2}}{n}+\frac{\left|J_{p}\right|^{2}}{p}+R_{n} \ln \left(\frac{n_{t r} n_{0} \mu_{n}}{n\left(1-n_{t r}\right)}\right)\right. \\
& \left.+R_{p} \ln \left(\frac{\left(1-n_{t r}\right) p_{0} \mu_{p}}{p n_{t r}}\right)\right) d x \geq 0 .
\end{aligned}
$$

The entropy production functional consists of two non-negative flux terms and two equally non-negative reaction terms of the form $(a-1) \ln a \geq 0$. Thus, the entropy $E$ and its production $P$ are non-negative functionals, which formally implies the entropy $E$ to be monotonically decreasing in time.

In a rigorous proof of the entropy decay, one has to control the two reaction terms in (7), which are unbounded for $n_{t r}(t, x) \rightarrow 0,1$ or $n(t, x), p(t, x) \rightarrow 0$. Hence the entropy production is potentially unbounded even for smooth solutions.

The following Theorem 1.1 comprises sufficient existence and regularity results for solutions to satisfy the weak version of (6). We shall call a global weak solution to system (1)-(4) a triple $\left(n, p, n_{t r}\right):[0, \infty) \rightarrow H^{1}(\Omega)^{2} \times L^{\infty}(\Omega)$ such that, first,

$$
n, p \in W_{2}(0, T):=\left\{f \in L^{2}\left((0, T), H^{1}(\Omega)\right) \mid \partial_{t} f \in L^{2}\left((0, T), H^{1}(\Omega)^{*}\right)\right\}
$$

for all $T \in(0, \infty)$ and, second, $\left(n, p, n_{t r}\right)$ solves (1) where $n$ and $p$ satisfy their dynamic equations and the boundary conditions (2) in the weak sense. From PDEtheory (see e.g. [3]), we further obtain the embedding $W_{2}(0, T) \hookrightarrow C\left([0, T], L^{2}(\Omega)\right)$.

Theorem 1.1 (Time-dependent system) Let $n_{0}, p_{0}, \tau_{n}, \tau_{p}$ and $\varepsilon$ be positive constants. Assume that $V_{n}$ and $V_{p}$ satisfy (4) and that $\Omega \subset \mathbb{R}^{m}, m \geq 1$, is a bounded, sufficiently smooth domain.

Then, for any non-negative initial datum $\left(n_{I}, p_{I}, n_{t r, I}\right) \in L^{\infty}(\Omega)^{3}$ satisfying $\left\|n_{t r, I}\right\|_{L^{\infty}(\Omega)} \leq 1$, there exists a unique non-negative global weak solution $\left(n, p, n_{t r}\right)$ of system (1) with boundary conditions (2). More precisely, we find that for all $T \in(0, \infty)$

$$
n, p \in W_{2}(0, T) \cap L^{\infty}\left((0, T), L^{\infty}(\Omega)\right),
$$

and

$$
n_{t r} \in C\left([0, T], L^{\infty}(\Omega)\right), \quad \partial_{t} n_{t r} \in C\left([0, T], L^{2}(\Omega)\right)
$$

Moreover, there exist positive constants $C_{n}\left(\left\|n_{I}\right\|_{L^{\infty}(\Omega)}, V_{n}\right), C_{p}\left(\left\|p_{I}\right\|_{L^{\infty}(\Omega)}, V_{p}\right)$ and $K_{n}\left(V_{n}\right), K_{p}\left(V_{p}\right)$ independent of $\varepsilon$ such that

$$
\|n(t, \cdot)\|_{L^{\infty}(\Omega)} \leq C_{n}+K_{n} t, \quad\|p(t, \cdot)\|_{L^{\infty}(\Omega)} \leq C_{p}+K_{p} t, \quad \text { for all } t \geq 0 .
$$


In addition, the concentration $n_{t r}(t, x)$ is bounded away from zero and one in the sense that for all times $\tau>0$ there exist positive constants $\eta=\eta\left(\varepsilon_{0}, \tau, \tau_{n}, \tau_{p}\right)$, $\theta=\theta\left(C_{n}, C_{p}, K_{n}, K_{p}\right)$ and a sufficiently small constant $\gamma\left(\tau, C_{n}, C_{p}, K_{n}, K_{p}\right)>0$ such that

$$
n_{t r}(t, x) \in\left[\min \left\{\eta t, \frac{\gamma}{1+\theta t}\right\}, \max \left\{1-\eta t, 1-\frac{\gamma}{1+\theta t}\right\}\right] \text { for all } t \geq 0 \text { and a.e. } x \in \Omega,
$$

where $\eta \tau=\frac{\gamma}{1+\theta \tau}$ such that the linear and the inverse linear bound intersect at time $\tau$. As a consequence of (12), there exist positive constants $\mu, \Gamma>0$ (depending on $\tau, \eta$, $\left.\theta, \gamma, V_{n}, V_{p}\right)$ such that

$$
n(t, x), p(t, x) \geq \min \left\{\mu \frac{t^{2}}{2}, \frac{\Gamma}{1+\theta t}\right\} \text { for all } t \geq 0 \text { and a.e. } x \in \Omega
$$

where $\mu \frac{\tau^{2}}{2}=\frac{\Gamma}{1+\theta \tau}$ such that the quadratic and the inverse linear bound intersect at the same time $\tau$.

Remark 1.2 (Proof of Theorem 1.1) The existence theory of Theorem 1.1 for the coupled ODE-PDE problem (1) applies standard parabolic methods and pointwise ODE estimates. It relates to previous results like [15] in assuming $L^{\infty}$ initial data and proving $L^{\infty}$-bounds in order to control non-linear terms. The proof is therefore postponed to the Appendix.

Our first main result proves exponential convergence of solutions to (1)-(4) to a unique positive equilibrium state $\left(n_{\infty}(x), p_{\infty}(x), n_{t r, \infty}\right)$, which is stated in detail in Theorem 2.1.

Theorem 1.3 (Exponential convergence to equilibrium) Let $\left(n, p, n_{t r}\right)$ be a global weak solution of system (1)-(4) as given in Theorem 1.1 above corresponding to the non-negative initial data $\left(n_{I}, p_{I}, n_{t r, I}\right) \in L^{\infty}(\Omega)^{3}$ satisfying $\left\|n_{t r, I}\right\|_{L^{\infty}(\Omega)} \leq 1$. Then, this solution satisfies the weak entropy production law

$$
E\left(n, p, n_{t r}\right)\left(t_{1}\right)+\int_{t_{0}}^{t_{1}} P\left(n, p, n_{t r}\right)(s) d s=E\left(n, p, n_{t r}\right)\left(t_{0}\right)
$$

for all $0<t_{0} \leq t_{1}<\infty$ and the following versions of the exponential decay towards the equilibrium:

$$
E\left(n, p, n_{t r}\right)(t)-E_{\infty} \leq\left(E_{I}-E_{\infty}\right) e^{-K t}
$$

where $E_{I}$ and $E_{\infty}$ denote the initial entropy and the equilibrium entropy of the system, respectively, and the equilibrium $\left(n_{\infty}, p_{\infty}, n_{t r, \infty}\right)$ is given in Theorem 2.1. Moreover, 


$$
\left\|n-n_{\infty}\right\|_{L^{1}(\Omega)}^{2}+\left\|p-p_{\infty}\right\|_{L^{1}(\Omega)}^{2}+\varepsilon\left\|n_{t r}-n_{t r, \infty}\right\|_{L^{2}(\Omega)}^{2} \leq C\left(E_{I}-E_{\infty}\right) e^{-K t}
$$

where $C:=C_{\mathrm{CKP}}^{-1}$ and $K:=C_{\mathrm{EEP}}^{-1}$ (see Theorem 1.5 and Proposition 6.1 for the definition of $C_{\mathrm{EEP}}$ and $C_{\mathrm{CKP}}$, respectively) are explicitly computable constants independent of $\varepsilon \in\left(0, \varepsilon_{0}\right]$ for arbitrary but fixed $\varepsilon_{0}>0$.

Remark 1.4 (Theorem 1.1 proves the weak entropy production law (14)) The regularity of $n$ and $p$ of Theorem 1.1 as well as the lower and upper bounds (12) for $n_{t r}$ and the lower bounds (13) for $n$ and $p$ allow to prove that any solution of Theorem 1.1 satisfies the weak entropy production law (14).

The proof of Theorem 1.3 applies the so-called entropy method, which derives a functional inequality of the form

$$
E\left(n, p, n_{t r}\right)-E\left(n_{\infty}, p_{\infty}, n_{t r, \infty}\right) \leq C P\left(n, p, n_{t r}\right),
$$

where $n, p$ and $n_{t r}$ are non-negative functions satisfying the same conservation law as solutions to (1)-(4), see below. The proof provides an explicit estimate of the constant $C>0$. Applying this entropy-entropy production (EEP) inequality to the entropy production law (14) entails exponential decay of the relative entropy via a general Gronwall-argument. A Csiszár-Kullback-Pinsker-type inequality yields then exponential convergence in $L^{1}$ as stated in (15).

The key step of the entropy method is to prove (as second main result) a suitable EEP functional inequality independently from solutions to (1)-(4) and independently from $\varepsilon$.

Theorem 1.5 (Entropy-Entropy Production Inequality) Let $\varepsilon_{0}, \tau_{n}, \tau_{p}, n_{0}, p_{0}$ be positive constants and $M \in \mathbb{R}$. Let $\left(n_{\infty}, p_{\infty}, n_{t r, \infty}\right)$ be the corresponding equilibrium as in Theorem 2.1. Consider an arbitrarily large positive constant $M_{1}>0$ and non-negative functions $\left(n, p, n_{t r}\right) \in L^{1}(\Omega)^{3}$ satisfying the $L^{1}$-bound $\bar{n}, \bar{p} \leq M_{1}$, the $L^{\infty}$-bound $\left\|n_{t r}\right\|_{L^{\infty}(\Omega)} \leq 1$, and the conservation law

$$
\bar{n}-\bar{p}+\varepsilon \overline{n_{t r}}=M
$$

where $\bar{f}:=\int_{\Omega} f(x) d x($ recall $|\Omega|=1)$.

Then, there exists an explicitly computable constant $C_{\mathrm{EEP}}>0$ such that for all $\varepsilon \in\left(0, \varepsilon_{0}\right]$ the following functional inequality, called entropy-entropy production inequality, holds true:

$$
E\left(n, p, n_{t r}\right)-E\left(n_{\infty}, p_{\infty}, n_{t r, \infty}\right) \leq C_{\mathrm{EEP}} P\left(n, p, n_{t r}\right) .
$$

Remark 1.6 We point out that Theorem 1.5 derives a general functional inequality for admissible functions $\left(n, p, n_{t r}\right)$, which only share few natural properties like the $L^{1}$-integrability, boundedness of the trapped states and the conservation law with solutions to (1)-(4). It is a nice robustness feature of the entropy method to be based on functional inequalities which can be reused in related contexts, rather than 
deriving solution-specific estimates. The constant $C_{\mathrm{EEP}}$ is independent of $\varepsilon \in\left(0, \varepsilon_{0}\right]$. It only depends on the upper bound $\varepsilon_{0}>0$, which can be chosen arbitrarily.

Remark 1.7 We emphasise that the EEP inequality (16) does not depend on the lower and upper solution bounds (11)-(13). These bounds are only needed to prove that solutions to (1)-(4) satisfy the weak entropy production law (14), which is neither directly obvious nor part of the existence theory. Therefore, (14) implies that solutions to Theorem 1.1 may only feature singularities of $P$ at time zero due to a lacking regularity of the initial data or due to initial data $n_{t r, I}(x) \in[0,1]$, $n_{I}(x), p_{I}(x) \in[0, \infty)$.

The proof of the EEP-inequality of Theorem 1.5 captures in a certain sense the entire non-linear and global dynamics of system (1)-(4). Hence, its derivation is ought to be an involved task. A key step is the proof of a functional EEP-inequality for the special cases of spatially homogeneous concentrations, which fulfil the conservation law (3) and the $L^{1}$-bounds (cf. Proposition 5.3). This core estimate is then extended to the case of arbitrary concentrations satisfying the same assumptions in Proposition 5.5. This extension also forces one to bound $\sqrt{n}-\sqrt{n}, \sqrt{p}-\sqrt{p}$, and $\sqrt{n_{t r}}-\overline{\sqrt{n_{t r}}}$ in $L^{2}(\Omega)$ by the entropy production. Due to the diffusive part in the dynamical equations for $n$ and $p$, this is easily achieved for the expressions involving $n$ and $p$ by applying Poincaré's inequality (see the Proof of Theorem 1.5 in Sect. 6). However, this is not possible for $n_{t r}$ as no diffusion is acting on $n_{t r}$. On the other hand, $n_{t r}$ is subject to indirect diffusive effects, which allow for a control on $\sqrt{n_{t r}}-\overline{\sqrt{n_{t r}}}$ in terms of a suitable functional inequality. Indirect diffusive effects occur when a reversible reaction transfers diffusive behaviour from a diffusive species to a non-diffusive species. A first functional inequality which quantifies an indirect diffusion effect was proven in [4] with significant generalisations to reaction-diffusion systems in [8, 12], volume-surface reaction-diffusion systems [11] and reaction-diffusion systems with non-linear diffusion [13]. Here, the corresponding estimate is proven in Proposition 5.6 and might also be of independent interest.

Our two last results on system (1)-(4) combine the exponential convergence to equilibrium as proven in Theorem 1.3 with the solution bounds of Theorem 1.1. This entails uniform-in-time solution bounds for $n$ and $p$ as well as exponential convergence to equilibrium in $L^{\infty}(\Omega)$ for $n, p$, and $n_{t r}$. As opposed to (15), the convergence result for $n_{t r}$ in Corollary 1.9 holds true without the coefficient $\varepsilon$.

Corollary 1.8 The solutions $n$ and $p$ of Theorem 1.1 are uniformly-in-time bounded in $L^{\infty}$, i.e. there exists a constant $Z>0$ independent of $\varepsilon \in\left(0, \varepsilon_{0}\right]$ such that

$$
\|n(t, \cdot)\|_{L^{\infty}(\Omega)},\|p(t, \cdot)\|_{L^{\infty}(\Omega)} \leq Z \text { for all } t \geq 0 .
$$

Moreover, the bounds (17) allow to improve the bounds (12), (13) and to obtain uniform-in-time bounds in the sense that for all $\tau>0$, there exist sufficiently small and $\varepsilon$-independent constants $\eta, \gamma, \mu, \Gamma>0$ such that 


$$
n_{t r}(t, x) \in[\min \{\eta t, \gamma\}, \max \{1-\eta t, 1-\gamma\}]
$$

and

$$
n(t, x), p(t, x) \geq \min \left\{\mu \frac{t^{2}}{2}, \Gamma\right\}
$$

for all $t \geq 0$ and a.e. $x \in \Omega$ where $\eta t$ and $\gamma$ as well as $\mu t^{2} / 2$ and $\Gamma$ intersect at time $\tau>0$.

Corollary 1.9 Under the hypotheses of Theorem 1.3, there exist constants $0<C, K<\infty$ independent of $\varepsilon \in\left(0, \varepsilon_{0}\right]$ such that

$$
\left\|n-n_{\infty}\right\|_{L^{\infty}(\Omega)}+\left\|p-p_{\infty}\right\|_{L^{\infty}(\Omega)}+\left\|n_{t r}-n_{t r, \infty}\right\|_{L^{\infty}(\Omega)} \leq C e^{-K t}
$$

is valid for all $t \geq 0$.

The final topic of this paper considers the limit $\varepsilon \rightarrow 0$, which recovers the wellknown Shockley-Read-Hall drift-diffusion-recombination model (see [15, 18]):

$$
\begin{cases}\partial_{t} n=\nabla \cdot J_{n}(n)+R(n, p), & J_{n}=\nabla n+n \nabla V_{n}, \\ \partial_{t} p=\nabla \cdot J_{p}(p)+R(n, p), & J_{p}=\nabla p+p \nabla V_{p},\end{cases}
$$

where

$$
R(n, p)=\frac{1-\frac{n p}{n_{0} p_{0} \mu_{n} \mu_{p}}}{\tau_{n}\left(1+\frac{p}{p_{0} \mu_{p}}\right)+\tau_{p}\left(1+\frac{n}{n_{0} \mu_{n}}\right)}
$$

Remarking that the entropy-entropy production inequality derived in Theorem 1.5 holds uniformly in the fast-reaction parameter $0<\varepsilon \leq \varepsilon_{0}$, one intuitively expects the entropy method and the convergence result of Theorem 1.3 to extend to system (20). Here, we are interested to make this conjecture rigorous also in view of a better general understanding of the equilibration of systems which are derived as fast-reaction limits or quasi-steady-state approximations. One technical point is how to bypass the $\varepsilon$-dependency of the conservation law (3). The details of this singular limit are subject of the last Sect. 7. Altogether, we prove for system (20) the Theorems 7.3, 7.2 and Corollary 7.5 as corresponding versions of Theorems 1.3, 1.5 and Corollary 1.8.

Up to our knowledge, this is a first result in performing the entropy method in a non-linear reaction-diffusion-type system uniformly in a fast-reaction limit. Note that our approach yields global convergence to equilibrium for all initial data rather than just exponential stability of equilibria as proven, for instance, in a related 1D Poisson-Nernst-Planck system uniformly in the permittivity entering Poisson's equation [17]. 
The rest of the paper is organised in the following manner. Section 2 proves the existence of a unique equilibrium (Theorem 2.1) as well as uniform-in- $\varepsilon$ bounds of $n_{\infty}, p_{\infty}$ and $n_{t r, \infty}$. In Sect. 3, we collect a couple of technical lemmata, and within Sect. 4, we state a preliminary proposition which serves as a first result towards an EEP-inequality. An abstract version of the EEP-estimate is proven in Sect. 5, first for constant concentrations and based on that also for general concentrations. Section 6 is concerned with the proofs of the EEP-inequality from Theorem 1.5, the announced exponential convergence from Theorem 1.3 and the uniform $L^{\infty}$-bounds from Corollary 1.8, whereas Sect. 7 is devoted to the same issues in the situation $\varepsilon \rightarrow 0$. Finally, the proof of Theorem 1.1 is contained in the Appendix.

\section{Properties of the equilibrium}

We prove the existence of a unique positive equilibrium $\left(n_{\infty}, p_{\infty}, n_{t r, \infty}\right)$ of system (1)-(3) in a suitable (and natural) function space. Note that uniqueness is only satisfied once the total charge $M$ in (3) is fixed. This equilibrium can either be seen as the unique solution of the below stationary system (21) or as the unique state for which the entropy production (7) vanishes.

Theorem 2.1 (Stationary system and uniformly bounded equilibrium) Let $M \in \mathbb{R}$, $\varepsilon \in\left(0, \varepsilon_{0}\right]$ for arbitrary $\varepsilon_{0}>0$ and $\left(n_{\infty}, p_{\infty}, n_{t r, \infty}\right) \in X$ where $X$ is defined via

$$
\begin{aligned}
X:=\left\{\left(n, p, n_{t r}\right) \in H^{1}(\Omega)^{2} \times L^{\infty}(\Omega) \mid \bar{n}-\bar{p}+\varepsilon \overline{n_{t r}}=M\right. \\
\left.\wedge(\exists \gamma>0) n, p \geq \gamma \text { a.e. } \wedge n_{t r} \in[\gamma, 1-\gamma] \text { a.e. }\right\} .
\end{aligned}
$$

Then, the following statements are equivalent.

1. $\left(n_{\infty}, p_{\infty}, n_{t r, \infty}\right) \in X$ is a solution of the stationary system

$$
\begin{gathered}
\nabla \cdot J_{n}\left(n_{\infty}\right)+R_{n}\left(n_{\infty}, n_{t r, \infty}\right)=0, \\
\nabla \cdot J_{p}\left(p_{\infty}\right)+R_{p}\left(p_{\infty}, n_{t r, \infty}\right)=0, \\
R_{p}\left(p_{\infty}, n_{t r, \infty}\right)-R_{n}\left(n_{\infty}, n_{t r, \infty}\right)=0 .
\end{gathered}
$$

2. $P\left(n_{\infty}, p_{\infty}, n_{t r, \infty}\right)=0$.

3. $J_{n}\left(n_{\infty}\right)=J_{p}\left(p_{\infty}\right)=R_{n}\left(n_{\infty}, n_{t r, \infty}\right)=R_{p}\left(p_{\infty}, n_{t r, \infty}\right)=0$ a.e. in $\Omega$.

4. The state $\left(n_{\infty}, p_{\infty}, n_{t r, \infty}\right)$ satisfies

$$
n_{\infty}=n_{*} e^{-V_{n}}, \quad p_{\infty}=p_{*} e^{-V_{p}}, \quad n_{t r, \infty}=\frac{n_{*}}{n_{*}+n_{0}}=\frac{p_{0}}{p_{*}+p_{0}}
$$

where the positive constants $n_{*}, p_{*}>0$ are uniquely determined by the condition 


$$
n_{*} p_{*}=n_{0} p_{0}
$$

and the conservation law

$$
n_{*} \overline{\mu_{n}}-p_{*} \overline{\mu_{p}}+\varepsilon n_{t r, \infty}=M .
$$

Consequently, the unique positive equilibrium $\left(n_{\infty}, p_{\infty}, n_{t r, \infty}\right) \in X$ is given by (22)-(24), and

$$
n_{t r, \infty}=\frac{n_{*}}{n_{0}}\left(1-n_{t r, \infty}\right), \quad 1-n_{t r, \infty}=\frac{p_{*}}{p_{0}} n_{t r, \infty} .
$$

Finally, for all $M \in \mathbb{R}$ and for $\varepsilon \in\left(0, \varepsilon_{0}\right]$, there exist two constants $\gamma \in(0,1 / 2)$ and $\Gamma \in(1 / 2, \infty)$ depending only on $\varepsilon_{0}, n_{0}, p_{0}, M, V:=\max \left(\left\|V_{n}\right\|_{L^{\infty}(\Omega)},\left\|V_{p}\right\|_{L^{\infty}(\Omega)}\right)$ such that

$$
\begin{aligned}
& n_{\infty}(x), p_{\infty}(x) \in[\gamma, \Gamma] \quad \text { for a.e. } x \in \Omega \text { and } \\
& n_{*}, p_{*} \in[\gamma, \Gamma], \quad n_{t r, \infty} \in[\gamma, 1-\gamma] .
\end{aligned}
$$

Proof of Theorem 2.1 We shall prove the equivalence of the statements in the theorem by a circular reasoning. Assume that $\left(n_{\infty}, p_{\infty}, n_{t r, \infty}\right) \in X$ is a solution of the stationary system (21). In this case,

$$
J_{n}\left(n_{\infty}\right), J_{p}\left(p_{\infty}\right), R_{n}\left(n_{\infty}, n_{t r, \infty}\right), R_{p}\left(p_{\infty}, n_{t r, \infty}\right) \in L^{2}(\Omega) .
$$

We test Eq. (21a) with $\ln \left(n_{\infty} /\left(n_{0} \mu_{n}\right)\right)$. Due to $n_{\infty} \in H^{1}(\Omega)$ and $n_{\infty} \geq \gamma$ a.e. in $\Omega$, the test function $\ln \left(n_{\infty} /\left(n_{0} \mu_{n}\right)\right)$ belongs to $H^{1}(\Omega)$. We find

$$
0=\int_{\Omega}\left(\frac{\left|J_{n}\left(n_{\infty}\right)\right|^{2}}{n_{\infty}}-R_{n}\left(n_{\infty}, n_{t r, \infty}\right) \ln \left(\frac{n_{\infty}}{n_{0} \mu_{n}}\right)\right) d x .
$$

In the same way, we test Eq. (21b) with $\ln \left(p_{\infty} /\left(p_{0} \mu_{p}\right)\right) \in H^{1}(\Omega)$. This yields

$$
0=\int_{\Omega}\left(\frac{\left|J_{p}\left(p_{\infty}\right)\right|^{2}}{p_{\infty}}-R_{p}\left(p_{\infty}, n_{t r, \infty}\right) \ln \left(\frac{p_{\infty}}{p_{0} \mu_{p}}\right)\right) d x .
$$

Moreover, we multiply (21c) with $\ln \left(n_{t r, \infty} /\left(1-n_{t r, \infty}\right)\right) \in L^{2}(\Omega)$, integrate over $\Omega$ and obtain

$$
0=\int_{\Omega}\left(\left(R_{n}\left(n_{\infty}, n_{t r, \infty}\right)-R_{p}\left(p_{\infty}, n_{t r, \infty}\right)\right) \ln \left(\frac{n_{t r, \infty}}{1-n_{t r, \infty}}\right)\right) d x .
$$

Taking the sum of the three expressions above, we arrive at 


$$
\begin{aligned}
P\left(n_{\infty}, p_{\infty}, n_{t r, \infty}\right)= & \int_{\Omega}\left(\frac{\left|J_{n}\left(n_{\infty}\right)\right|^{2}}{n_{\infty}}+\frac{\left|J_{p}\left(p_{\infty}\right)\right|^{2}}{p_{\infty}}\right. \\
& -\underbrace{R_{n}\left(n_{\infty}, n_{t r, \infty}\right) \ln \left(\frac{n_{\infty}\left(1-n_{t r, \infty}\right)}{n_{0} \mu_{n} n_{t r, \infty}}\right)}_{\leq 0} \\
& -\underbrace{R_{p}\left(p_{\infty}, n_{t r, \infty}\right) \ln \left(\frac{p_{\infty} n_{t r, \infty}}{p_{0} \mu_{p}\left(1-n_{t r, \infty}\right)}\right)}_{\leq 0}) d x=0 .
\end{aligned}
$$

By the non-negativity of the terms in the last two lines, equality holds if and only if $R_{n}\left(n_{\infty}, n_{t r, \infty}\right)=0=R_{p}\left(p_{\infty}, n_{t r, \infty}\right)$. Hence, $P\left(n_{\infty}, p_{\infty}, n_{t r, \infty}\right)=0$ readily implies $J_{n}\left(n_{\infty}\right)=J_{p}\left(p_{\infty}\right)=R_{n}\left(n_{\infty}, n_{t r, \infty}\right)=R_{p}\left(p_{\infty}, n_{t r, \infty}\right)=0$ a.e. in $\Omega$.

Because of $J_{n}\left(n_{\infty}\right)=\mu_{n} \nabla\left(\frac{n_{\infty}}{\mu_{n}}\right)=0=J_{p}\left(p_{\infty}\right)=\mu_{p} \nabla\left(\frac{p_{\infty}}{\mu_{p}}\right)$, we know that

$$
n_{\infty}(x)=n_{*} e^{-V_{n}}, \quad p_{\infty}(x)=p_{*} e^{-V_{p}}
$$

with constants $n_{*}, p_{*}$. Moreover, $R_{n}\left(n_{\infty}, n_{t r, \infty}\right)=R_{p}\left(p_{\infty}, n_{t r, \infty}\right)=0$ gives rise to

$$
n_{t r, \infty}=\frac{n_{*}}{n_{0}}\left(1-n_{t r, \infty}\right), \quad 1-n_{t r, \infty}=\frac{p_{*}}{p_{0}} n_{t r, \infty} .
$$

Consequently, $n_{*} p_{*}=n_{0} p_{0}>0$, which implies $n_{*}, p_{*}>0$ and

$$
n_{t r, \infty}=\frac{n_{*}}{n_{*}+n_{0}}=\frac{p_{0}}{p_{*}+p_{0}} \in(0,1) .
$$

Moreover, for $M$ fixed, the constants $n_{*}$ and $p_{*}$ are uniquely determined by the conservation law

$$
n_{*} \overline{\mu_{n}}-p_{*} \overline{\mu_{p}}+\varepsilon n_{t r, \infty}=M,
$$

where the uniqueness follows from the strict monotonicity of $f\left(n_{*}\right):=n_{*} \overline{\mu_{n}}-\frac{n_{0} p_{0}}{n_{*}} \overline{\mu_{p}}+\varepsilon \frac{n_{*}}{n_{*}+n_{0}}$ on $(0, \infty)$ and the asymptotics $f\left(n_{*}\right) \rightarrow-\infty$ for $n_{*} \rightarrow 0^{+}$and $f\left(n_{*}\right) \rightarrow \infty$ for $n_{*} \rightarrow \infty$.

Finally, concluding the circular reasoning $1 . \rightarrow 2 . \rightarrow 3 . \rightarrow 4 . \rightarrow 1$., we observe that

$$
n_{\infty}=n_{*} e^{-V_{n}}, \quad p_{\infty}=p_{*} e^{-V_{p}}, \quad n_{t r, \infty}=\frac{n_{*}}{n_{*}+n_{0}}=\frac{p_{0}}{p_{*}+p_{0}}
$$

obviously satisfies $J_{n}\left(n_{\infty}\right)=J_{p}\left(p_{\infty}\right)=R_{n}\left(n_{\infty}, n_{t r, \infty}\right)=R_{p}\left(p_{\infty}, n_{t r, \infty}\right)=0$ a.e. in $\Omega$ which proves $\left(n_{\infty}, p_{\infty}, n_{t r, \infty}\right)$ to be a solution of the stationary system.

In order to prove the bounds (26), we observe

$$
n_{*} \overline{\mu_{n}}-\frac{n_{0} p_{0}}{n_{*}} \overline{\mu_{p}}=M-\varepsilon n_{t r, \infty}=M-\varepsilon \frac{n_{*}}{n_{*}+n_{0}}
$$


the left hand side is strictly monotone increasing from $-\infty$ to $+\infty$ as $n_{*} \in(0, \infty)$, while the right hand side is strictly monotone decreasing and bounded between $\left(M, M-\varepsilon_{0}\right)$ as $n_{*} \in(0, \infty)$. Both monotonicity and unboundedness/boundedness imply uniform positive lower and upper bounds for $n_{*}$ as explicitly proven in the following: First, we derive that

$$
n_{*}=\frac{M-\varepsilon n_{t r, \infty}}{2 \overline{\mu_{n}}}+\sqrt{\frac{\left(M-\varepsilon n_{t r, \infty}\right)^{2}}{4 \bar{\mu}_{n}^{2}}+\frac{n_{0} p_{0} \overline{\mu_{p}}}{\overline{\mu_{n}}}}>0
$$

for all $\varepsilon \in\left(0, \varepsilon_{0}\right]$. Note that (27) is not an explicit representation of $n_{*}$ since $n_{t r, \infty}$ depends itself on $n_{*}$. Because of $n_{t r, \infty} \in(0,1)$, we further observe that

$$
\begin{aligned}
n_{*} & \leq \frac{\left|M-\varepsilon n_{t r, \infty}\right|}{2 \overline{\mu_{n}}}+\sqrt{\frac{\left(M-\varepsilon n_{t r, \infty}\right)^{2}}{4 \bar{\mu}_{n}^{2}}}+\sqrt{\frac{n_{0} p_{0} \overline{\mu_{p}}}{\overline{\mu_{n}}}} \\
& \leq \frac{|M|+\varepsilon_{0}}{\overline{\mu_{n}}}+\sqrt{\frac{n_{0} p_{0} \overline{\mu_{p}}}{\overline{\mu_{n}}}} \leq \beta<\infty,
\end{aligned}
$$

where $\beta=\beta\left(\varepsilon_{0}, n_{0}, p_{0}, M, V\right)$. And as a result of the elementary inequality $\sqrt{a+b} \geq \sqrt{a}+\frac{b}{2 \sqrt{a}+\sqrt{b}}$ for $a \geq 0$ and $b>0$, we also conclude that

$$
\begin{aligned}
n_{*} & \geq \frac{M-\varepsilon n_{t r, \infty}}{2 \overline{\mu_{n}}}+\frac{\left|M-\varepsilon n_{t r, \infty}\right|}{2 \overline{\mu_{n}}}+\frac{\frac{n_{0} p_{0} \overline{\mu_{p}}}{\overline{\mu_{n}}}}{\frac{\left|M-\varepsilon n_{t r, \infty}\right|}{\overline{\mu_{n}}}+\sqrt{\frac{n_{0} p_{0} \overline{\mu_{p}}}{\overline{\mu_{n}}}}} \\
& \geq \frac{\frac{n_{0} p_{0} \overline{\mu_{p}}}{\overline{\mu_{n}}}}{\frac{|M|+\varepsilon_{0}}{\overline{\mu_{n}}}+\sqrt{\frac{n_{0} p_{0} \overline{\mu_{p}}}{\overline{\mu_{n}}}}} \geq \alpha>0
\end{aligned}
$$

where $\alpha=\alpha\left(\varepsilon_{0}, n_{0}, p_{0}, M, V\right)$. Similar arguments show that corresponding bounds $\alpha$ and $\beta$ are also available for $p_{*}$. Hence,

$$
n_{t r, \infty} \in\left[\frac{\alpha}{\alpha+n_{0}}, \frac{\beta}{\beta+n_{0}}\right] \text {. }
$$

Due to $n_{\infty}=n_{*} e^{-V_{n}}, p_{\infty}=p_{*} e^{-V_{p}}$ and the $L^{\infty}$-bounds on $V_{n}$ and $V_{p}$, the claim of the proposition follows.

\section{Some technical lemmata}

A particularly useful relation between the concentrations $n, p$ and $n_{t r}$ is the following Lemma. 
Lemma 3.1 The conservation law $\bar{n}-\bar{p}+\varepsilon \overline{n_{t r}}=M$ and the equilibrium condition (25) imply

$$
\begin{aligned}
\forall t & \geq 0: \quad\left(\bar{n}-\overline{n_{\infty}}\right) \ln \left(\frac{n_{*}}{n_{0}}\right)+\left(\bar{p}-\overline{p_{\infty}}\right) \ln \left(\frac{p_{*}}{p_{0}}\right) \\
& =\varepsilon\left(\overline{n_{t r}}-n_{t r, \infty}\right) \ln \left(\frac{1-n_{t r, \infty}}{n_{t r, \infty}}\right) .
\end{aligned}
$$

Proof With $\overline{n_{\infty}}-\overline{p_{\infty}}+\varepsilon n_{t r, \infty}=M$ (note that $n_{t r, \infty}=\overline{n_{t r, \infty}}$ is constant), we have $\bar{p}-\overline{p_{\infty}}=\bar{n}-\overline{n_{\infty}}+\varepsilon\left(\overline{n_{t r}}-n_{t r, \infty}\right)$. We employ this relation to replace $\bar{p}-\overline{p_{\infty}}$ on the left hand side of (28) and calculate

$$
\begin{aligned}
(\bar{n} & \left.-\overline{n_{\infty}}\right) \ln \left(\frac{n_{*}}{n_{0}}\right)+\left(\bar{p}-\overline{p_{\infty}}\right) \ln \left(\frac{p_{*}}{p_{0}}\right) \\
& =\left(\bar{n}-\overline{n_{\infty}}\right) \ln \left(\frac{n_{*} p_{*}}{n_{0} p_{0}}\right)+\varepsilon\left(\overline{n_{t r}}-n_{t r, \infty}\right) \ln \left(\frac{p_{*}}{p_{0}}\right) .
\end{aligned}
$$

Now, the first term on the right hand side vanishes due to $n_{*} p_{*}=n_{0} p_{0}$ while we use $p_{*} / p_{0}=\left(1-n_{t r, \infty}\right) / n_{t r, \infty}$ for the second term and obtain

$$
\left(\bar{n}-\overline{n_{\infty}}\right) \ln \left(\frac{n_{*}}{n_{0}}\right)+\left(\bar{p}-\overline{p_{\infty}}\right) \ln \left(\frac{p_{*}}{p_{0}}\right)=\varepsilon\left(\overline{n_{t r}}-n_{t r, \infty}\right) \ln \left(\frac{1-n_{t r, \infty}}{n_{t r, \infty}}\right)
$$

as claimed above.

Lemma 3.2 (Relative Entropy) The entropy relative to the equilibrium reads

$$
\begin{aligned}
& E\left(n, p, n_{t r}\right)-E\left(n_{\infty}, p_{\infty}, n_{t r, \infty}\right) \\
& =\int_{\Omega}\left(n \ln \frac{n}{n_{\infty}}-\left(n-n_{\infty}\right)+p \ln \frac{p}{p_{\infty}}-\left(p-p_{\infty}\right)\right. \\
& \left.\quad+\varepsilon \int_{n_{t r, \infty}}^{n_{t r}(x)}\left(\ln \left(\frac{s}{1-s}\right)-\ln \left(\frac{n_{t r, \infty}}{1-n_{t r, \infty}}\right)\right) d s\right) d x .
\end{aligned}
$$

Proof By the definition of $E\left(n, p, n_{t r}\right)$ in (5), we note that

$$
\begin{aligned}
& E\left(n, p, n_{t r}\right)-E\left(n_{\infty}, p_{\infty}, n_{t r, \infty}\right) \\
& =\int_{\Omega}\left(n \ln \left(\frac{n}{n_{0} \mu_{n}}\right)-n_{\infty} \ln \left(\frac{n_{\infty}}{n_{0} \mu_{n}}\right)-\left(n-n_{\infty}\right)\right. \\
& \quad+p \ln \left(\frac{p}{p_{0} \mu_{p}}\right)-p_{\infty} \ln \left(\frac{p_{\infty}}{p_{0} \mu_{p}}\right)-\left(p-p_{\infty}\right) \\
& \left.\quad+\varepsilon \int_{n_{t r, \infty}}^{n_{t r}(x)} \ln \left(\frac{s}{1-s}\right) d s\right) d x .
\end{aligned}
$$


We expand the first integrand as $n \ln \left(\frac{n}{n_{0} \mu_{n}}\right)=n \ln \left(\frac{n}{n_{\infty}}\right)+n \ln \left(\frac{n_{\infty}}{n_{0} \mu_{n}}\right)$. Thus, with $n_{\infty} / \mu_{n}=n_{*}$, we get

$$
\begin{aligned}
\int_{\Omega} & \left(n \ln \left(\frac{n}{n_{0} \mu_{n}}\right)-n_{\infty} \ln \left(\frac{n_{\infty}}{n_{0} \mu_{n}}\right)-\left(n-n_{\infty}\right)\right) d x \\
= & \int_{\Omega}\left(n \ln \left(\frac{n}{n_{\infty}}\right)-\left(n-n_{\infty}\right)\right) d x+\left(\bar{n}-\overline{n_{\infty}}\right) \ln \left(\frac{n_{*}}{n_{0}}\right) .
\end{aligned}
$$

Together with an analogous calculation of the $p$-terms, we obtain

$$
\begin{aligned}
& E\left(n, p, n_{t r}\right)-E\left(n_{\infty}, p_{\infty}, n_{t r, \infty}\right) \\
& =\int_{\Omega}\left(n \ln \left(\frac{n}{n_{\infty}}\right)-\left(n-n_{\infty}\right)+p \ln \left(\frac{p}{p_{\infty}}\right)-\left(p-p_{\infty}\right)\right) d x \\
& \quad+\left(\bar{n}-\overline{n_{\infty}}\right) \ln \left(\frac{n_{*}}{n_{0}}\right)+\left(\bar{p}-\overline{p_{\infty}}\right) \ln \left(\frac{p_{*}}{p_{0}}\right) \\
& \quad+\varepsilon \int_{\Omega} \int_{n_{t r, \infty}}^{n_{t r}(x)} \ln \left(\frac{s}{1-s}\right) d s d x .
\end{aligned}
$$

Lemma 3.1 allows us to reformulate the last two lines as

$$
\begin{aligned}
(\bar{n} & \left.-\overline{n_{\infty}}\right) \ln \left(\frac{n_{*}}{n_{0}}\right)+\left(\bar{p}-\overline{p_{\infty}}\right) \ln \left(\frac{p_{*}}{p_{0}}\right)+\varepsilon \int_{\Omega} \int_{n_{t r, \infty}}^{n_{t r}(x)} \ln \left(\frac{s}{1-s}\right) d s d x \\
= & \varepsilon\left(\overline{n_{t r}}-n_{t r, \infty}\right) \ln \left(\frac{1-n_{t r, \infty}}{n_{t r, \infty}}\right)+\varepsilon \int_{\Omega} \int_{n_{t r, \infty}}^{n_{t r}(x)} \ln \left(\frac{s}{1-s}\right) d s d x \\
= & \varepsilon \int_{\Omega} \int_{n_{t r, \infty}}^{n_{t r}(x)}\left(\ln \left(\frac{s}{1-s}\right)-\ln \left(\frac{n_{t r, \infty}}{1-n_{t r, \infty}}\right)\right) d s d x,
\end{aligned}
$$

which proves the claim.

Lemma 3.3 (Csiszár-Kullback-Pinsker inequality) Let $f, g: \Omega \rightarrow \mathbb{R}$ be non-negative measurable functions. Then,

$$
\int_{\Omega}\left(f \ln \left(\frac{f}{g}\right)-(f-g)\right) d x \geq \frac{3}{2 \bar{f}+4 \bar{g}}\|f-g\|_{L^{1}(\Omega)}^{2} .
$$

Proof Following a proof by Pinsker, we start with the elementary inequality $3(x-1)^{2} \leq(2 x+4)(x \ln x-(x-1))$. This allows us to derive the following CsiszárKullback-Pinsker-type inequality: 


$$
\begin{aligned}
\|f-g\|_{L^{1}(\Omega)} & =\int_{\Omega} g\left|\frac{f}{g}-1\right| d x \leq \int_{\Omega} g \sqrt{\frac{2}{3} \frac{f}{g}+\frac{4}{3}} \sqrt{\frac{f}{g} \ln \left(\frac{f}{g}\right)-\left(\frac{f}{g}-1\right)} d x \\
& =\int_{\Omega} \sqrt{\frac{2}{3} f+\frac{4}{3}} g \sqrt{f \ln \left(\frac{f}{g}\right)-(f-g) d x} \\
& \leq \sqrt{\frac{2}{3} \bar{f}+\frac{4}{3} \bar{g}} \sqrt{\int_{\Omega}\left(f \ln \left(\frac{f}{g}\right)-(f-g)\right) d x}
\end{aligned}
$$

where we applied Hölder's inequality in the last step.

The subsequent lemma provides $L^{1}$-bounds for $n$ and $p$ in terms of the initial entropy of the system and some further constants.

Lemma 3.4 ( $L^{1}$-bounds) Due to the monotonicity of the entropy functional, any entropy producing solution of (1) satisfies

$$
\forall t \geq 0: \quad \bar{n}, \bar{p} \leq \frac{5}{2} \max \left\{n_{0} \overline{\mu_{n}}, p_{0} \overline{\mu_{p}}\right\}+\frac{3}{4} E\left(n(0), p(0), n_{t r}(0)\right)=: M_{1}
$$

Proof Employing Lemma 3.3 and Young's inequality, we find

$$
\begin{aligned}
\bar{n} & \leq n_{0} \overline{\mu_{n}}+\left\|n-n_{0} \mu_{n}\right\|_{L^{1}(\Omega)} \\
& \leq n_{0} \overline{\mu_{n}}+\sqrt{\frac{2}{3} \bar{n}+\frac{4}{3} n_{0} \overline{\mu_{n}}} \sqrt{\int_{\Omega}\left(n \ln \left(\frac{n}{n_{0} \mu_{n}}\right)-\left(n-n_{0} \mu_{n}\right)\right) d x} \\
& \leq n_{0} \overline{\mu_{n}}+\frac{1}{3} \bar{n}+\frac{2}{3} n_{0} \overline{\mu_{n}}+\frac{1}{2} \int_{\Omega}\left(n \ln \left(\frac{n}{n_{0} \mu_{n}}\right)-\left(n-n_{0} \mu_{n}\right)\right) d x .
\end{aligned}
$$

Solving this inequality for $\bar{n}$ yields

$$
\bar{n} \leq \frac{5}{2} n_{0} \overline{\mu_{n}}+\frac{3}{4} \int_{\Omega}\left(n \ln \left(\frac{n}{n_{0} \mu_{n}}\right)-\left(n-n_{0} \mu_{n}\right)\right) d x .
$$

Therefore, we arrive at

$$
\bar{n} \leq \frac{5}{2} n_{0} \overline{\mu_{n}}+\frac{3}{4} E\left(n, p, n_{t r}\right) \leq \frac{5}{2} \max \left\{n_{0} \overline{\mu_{n}}, p_{0} \overline{\mu_{p}}\right\}+\frac{3}{4} E\left(n(0), p(0), n_{t r}(0)\right)
$$

where we used the monotonicity of the entropy functional in the last step. In the same way, we may bound $\bar{p}$ from above.

At certain points, we will have to estimate the difference between terms like $\overline{n / n_{\infty}}$ and $\bar{n} / \overline{n_{\infty}}$. Using Lemma 3.5 below, we can bound this difference by the $J_{n^{-}}$ flux term and, hence, by the entropy production. 
Lemma 3.5 Let $f \in L^{1}(\Omega)$ and $g \in L^{\infty}(\Omega)$ such that $f \geq 0, g \geq \gamma>0$ a.e. on $\Omega$ and flg is weakly differentiable. Then, there exists an explicit constant $C\left(\|f\|_{L^{1}(\Omega)},\|g\|_{L^{\infty}(\Omega)}, \gamma\right)>0$ such that

$$
\left(\frac{\bar{f}}{\bar{g}}-\overline{\left(\frac{f}{g}\right)}\right)^{2} \leq C \int_{\Omega}\left|\nabla \sqrt{\frac{f}{g}}\right|^{2} d x
$$

Proof We define $\delta:=\frac{f}{g}-\overline{\left(\frac{f}{g}\right)}$ and obtain $f=g\left(\overline{\left(\frac{f}{g}\right)}+\delta\right)$ and

$$
\frac{\bar{f}}{\bar{g}}=\int_{\Omega} \frac{f}{\bar{g}} d x=\int_{\Omega} \frac{g}{\bar{g}}\left(\overline{\left(\frac{f}{g}\right)}+\delta\right) d x=\overline{\left(\frac{f}{g}\right)}+\int_{\Omega} \frac{g}{\bar{g}} \delta d x
$$

by utilising $|\Omega|=1$. Therefore,

$$
\left|\frac{\bar{f}}{\bar{g}}-\overline{\left(\frac{f}{g}\right)}\right| \leq \frac{\|g\|_{L^{\infty}(\Omega)}}{\bar{g}}\|\delta\|_{L^{1}(\Omega)} \leq C_{P} \frac{\|g\|_{L^{\infty}(\Omega)}}{\bar{g}}\left\|\nabla\left(\frac{f}{g}\right)\right\|_{L^{1}(\Omega)}
$$

by applying Poincaré's inequality to $\delta$ with $\bar{\delta}=0$ and some constant $C_{P}(\Omega)>0$. As $g \geq \gamma>0$ is uniformly positive on $\Omega$ and $\bar{g} \geq \gamma$, we arrive at

$$
\left|\frac{\bar{f}}{\bar{g}}-\overline{\left(\frac{f}{g}\right)}\right| \leq C_{P} \frac{\|g\|_{L^{\infty}(\Omega)}}{\gamma^{2}}\left\|g \nabla\left(\frac{f}{g}\right)\right\|_{L^{1}(\Omega)} .
$$

Finally, we deduce

$$
\begin{aligned}
& \left(\frac{\bar{f}}{\bar{g}}-\overline{\left(\frac{f}{g}\right)}\right)^{2} \leq\left(C_{P} \frac{\|g\|_{L^{\infty}(\Omega)}}{\gamma^{2}}\right)^{2}\left\|\sqrt{f g} \sqrt{\frac{g}{f}} \nabla\left(\frac{f}{g}\right)\right\|_{L^{1}(\Omega)}^{2} \\
& \leq 4 \overline{f g}\left(C_{P} \frac{\|g\|_{L^{\infty}(\Omega)}}{\gamma^{2}}\right)^{2} \int_{\Omega}\left|\nabla \sqrt{\frac{f}{g}}\right|^{2} d x
\end{aligned}
$$

employing Hölder's inequality in the second step.

\section{Two preliminary propositions}

Notation 4.1 For arbitrary functions $f$, we define the normalised quantity

$$
\tilde{f}:=\frac{f}{\bar{f}} .
$$

The following logarithmic Sobolev inequality on bounded domains was proven in [6] by following an argument of Stroock [22]. 
Lemma 4.2 (Logarithmic Sobolev inequality on bounded domains) Let $\Omega$ be a bounded domain in $\mathbb{R}^{m}, m \geq 1$, such that the Poincaré (-Wirtinger) and Sobolev inequalities

$$
\begin{gathered}
\left\|\phi-\int_{\Omega} \phi d x\right\|_{L^{2}(\Omega)}^{2} \leq P(\Omega)\|\nabla \phi\|_{L^{2}(\Omega)}^{2}, \\
\|\phi\|_{L^{q}(\Omega)}^{2} \leq C_{1}(\Omega)\|\nabla \phi\|_{L^{2}(\Omega)}^{2}+C_{2}(\Omega)\|\phi\|_{L^{2}(\Omega)}^{2}, \quad \frac{1}{q}=\frac{1}{2}-\frac{1}{m}, \quad m \geq 3,
\end{gathered}
$$

hold with $q=\infty$ for $m=1$ and any $q<\infty$ for $m=2$. Then, the logarithmic Sobolev inequality

$$
\int_{\Omega} \phi^{2} \ln \left(\frac{\phi^{2}}{\|\phi\|_{L^{2}(\Omega)}^{2}}\right) d x \leq L(\Omega, m)\|\nabla \phi\|_{L^{2}(\Omega)}^{2}
$$

holds (for some constant $L(\Omega, m)>0)$.

The Log-Sobolev inequality allows to bound an appropriate part of the entropy functional by the flux parts of the entropy production. The normalised variables on the left hand side of the subsequent inequality naturally arise when reformulating the flux terms on the right hand side in such a way that we can apply the LogSobolev inequality on $\Omega$.

Proposition 4.3 Recall the assumptions $\left\|V_{n}\right\|_{L^{\infty}(\Omega)},\left\|V_{p}\right\|_{L^{\infty}(\Omega)} \leq V$. Then, there exists a constant $C(V)>0$ such that

$$
\int_{\Omega}\left(n \ln \left(\frac{\tilde{n}}{\widetilde{\mu_{n}}}\right)+p \ln \left(\frac{\tilde{p}}{\widetilde{\mu_{p}}}\right)\right) d x \leq C \int_{\Omega}\left(\frac{\left|J_{n}\right|^{2}}{n}+\frac{\left|J_{p}\right|^{2}}{p}\right) d x .
$$

Proof From the definition of $J_{n}$ one obtains

$$
\begin{aligned}
\int_{\Omega} \frac{\left|J_{n}\right|^{2}}{n} d x & =\int_{\Omega} \frac{\mu_{n}}{n}\left|\nabla\left(\frac{n}{\mu_{n}}\right)\right|^{2} \mu_{n} d x=4 \bar{n} \int_{\Omega} \frac{\mu_{n}}{\bar{n}}\left|\nabla \sqrt{\frac{n}{\mu_{n}}}\right|^{2} d x \\
& =4 \bar{n} \int_{\Omega} \frac{\mu_{n}}{\overline{\mu_{n}}}\left|\nabla \sqrt{\frac{\tilde{n}}{\tilde{\mu_{n}}}}\right|^{2} d x .
\end{aligned}
$$

We set

$$
\phi(x):=\sqrt{\frac{\tilde{n}}{\widetilde{\mu}_{n}}}, \quad \alpha:=\int_{\Omega} \phi(x)^{2} d x
$$

and observe that $\alpha=\frac{\overline{\mu_{n}}}{\bar{n}} \int_{\Omega} \frac{n}{\mu_{n}} d x \leq \overline{\mu_{n}} e^{V}$ is bounded independently of $n$. Next, we introduce the rescaled variable $y:=\alpha^{-\frac{1}{m}} x$ where $m \geq 1$ denotes the space dimension. 
Note that $\|\phi\|_{L^{2}(d x)}$ is in general different from one, whereas $\|\phi\|_{L^{2}(d y)}=1$. We now estimate with $\left\|V_{n}\right\|_{L^{\infty}(\Omega)} \leq V$ and the logarithmic Sobolev inequality (31)

$$
\begin{aligned}
\int_{\Omega}\left|\nabla_{x} \phi\right|^{2} d x & =\int_{\Omega}\left|\alpha^{-\frac{1}{m}} \nabla_{y} \phi\right|^{2} \alpha d y=\alpha^{1-\frac{2}{m}} \int_{\Omega}\left|\nabla_{y} \phi\right|^{2} d y \\
& \geq \alpha^{1-\frac{2}{m}} \frac{1}{L} \int_{\Omega} \phi^{2} \ln \left(\phi^{2}\right) d y=\alpha^{1-\frac{2}{m}} \frac{1}{L} \int_{\Omega} \frac{\tilde{n}}{\widetilde{\mu_{n}}} \ln \left(\frac{\tilde{n}}{\widetilde{\mu_{n}}}\right) d y \\
& =\alpha^{-\frac{2}{m}} \frac{1}{L} \frac{\overline{\mu_{n}}}{\bar{n}} \int_{\Omega} \frac{n}{\mu_{n}} \ln \left(\frac{\tilde{n}}{\widetilde{\mu_{n}}}\right) d x .
\end{aligned}
$$

The corresponding estimate involving $J_{n}$ reads

$$
\int_{\Omega} \frac{\left|J_{n}\right|^{2}}{n} d x \geq 4 \frac{\bar{n}}{\overline{\mu_{n}}} e^{-V} \int_{\Omega}\left|\nabla_{x} \phi\right|^{2} d x \geq \frac{4}{L} \alpha^{-\frac{2}{m}} e^{-2 V} \int_{\Omega} n \ln \left(\frac{\tilde{n}}{\widetilde{\mu}_{n}}\right) d x .
$$

The same arguments apply to the terms involving $p$.

The following proposition contains the first step towards an entropy-entropy production inequality. The relative entropy can be controlled by the flux part of the entropy production and three additional terms, which mainly consist of square roots of averaged quantities. The proof that the entropy production also serves as an upper bound for these terms will be the subject of the next section.

Proposition 4.4 There exists an explicit constant $C\left(\gamma, \Gamma, M_{1}\right)>0$ such that for $\left(n_{\infty}, p_{\infty}, n_{t r, \infty}\right) \in X$ from Theorem 2.1 and all non-negative functions $\left(n, p, n_{t r}\right) \in L^{1}(\Omega)^{3}$ satisfying $n_{t r} \leq 1$, the conservation law

$$
\bar{n}-\bar{p}+\varepsilon \overline{n_{t r}}=M
$$

and the $L^{1}$-bound

$$
\bar{n}, \bar{p} \leq M_{1}
$$

the following estimate holds true:

$$
\begin{aligned}
& E\left(n, p, n_{t r}\right)-E\left(n_{\infty}, p_{\infty}, n_{t r, \infty}\right) \leq C\left(\int_{\Omega}\left(\frac{\left|J_{n}\right|^{2}}{n}+\frac{\left|J_{p}\right|^{2}}{p}\right) d x\right. \\
& \left.\quad+\left(\sqrt{\overline{\left(\frac{n}{\mu_{n}}\right)}}-\sqrt{n_{*}}\right)^{2}+\left(\sqrt{\overline{\left(\frac{p}{\mu_{p}}\right)}}-\sqrt{p_{*}}\right)^{2}+\varepsilon \int_{\Omega}\left(\sqrt{n_{t r}}-\sqrt{n_{t r, \infty}}\right)^{2} d x\right) .
\end{aligned}
$$

(Note that the right hand side of (32) vanishes at the equilibrium $\left(n_{\infty}, p_{\infty}, n_{t r, \infty}\right)$.)

Proof According to Lemma 3.2, we have 


$$
\begin{aligned}
& E\left(n, p, n_{t r}\right)-E\left(n_{\infty}, p_{\infty}, n_{t r, \infty}\right) \\
& =\int_{\Omega}\left(n \ln \frac{n}{n_{\infty}}-\left(n-n_{\infty}\right)+p \ln \frac{p}{p_{\infty}}-\left(p-p_{\infty}\right)\right. \\
& \left.\quad+\varepsilon \int_{n_{t r, \infty}}^{n_{t r}}\left(\ln \left(\frac{s}{1-s}\right)-\ln \left(\frac{n_{t r, \infty}}{1-n_{t r, \infty}}\right)\right) d s\right) d x .
\end{aligned}
$$

Recall that $n=\tilde{n} \bar{n}, n_{\infty}=\widetilde{n_{\infty}} \overline{n_{\infty}}$ and $\widetilde{n_{\infty}}=\tilde{\mu_{n}}$. Using these relations, we rewrite the first two integrands as

$$
n \ln \left(\frac{n}{n_{\infty}}\right)-\left(n-n_{\infty}\right)=n \ln \left(\frac{\tilde{n}}{\widetilde{\mu}_{n}}\right)+n \ln \left(\frac{\bar{n}}{\overline{n_{\infty}}}\right)-\left(n-n_{\infty}\right)
$$

and analogously for the $p$-terms. This results in

$$
\begin{aligned}
E\left(n, p, n_{t r}\right)-E\left(n_{\infty}, p_{\infty}, n_{t r, \infty}\right) \\
=\int_{\Omega}\left(n \ln \left(\frac{\tilde{n}}{\widetilde{\mu_{n}}}\right)+p \ln \left(\frac{\tilde{p}}{\widetilde{\mu_{p}}}\right)\right) d x \\
\quad+\overline{n_{\infty}}\left(\frac{\bar{n}}{\overline{n_{\infty}}} \ln \left(\frac{\bar{n}}{\overline{n_{\infty}}}\right)-\left(\frac{\bar{n}}{\overline{n_{\infty}}}-1\right)\right) \\
\quad+\overline{p_{\infty}}\left(\frac{\bar{p}}{\overline{p_{\infty}}} \ln \left(\frac{\bar{p}}{\overline{p_{\infty}}}\right)-\left(\frac{\bar{p}}{\overline{p_{\infty}}}-1\right)\right) \\
\quad+\varepsilon \int_{\Omega} \int_{n_{t r, \infty}}^{n_{t r}}\left(\ln \left(\frac{s}{1-s}\right)-\ln \left(\frac{n_{t r, \infty}}{1-n_{t r, \infty}}\right)\right) d s d x .
\end{aligned}
$$

The terms in the second line of (33) can be estimated using the Log-Sobolev inequality of Proposition 4.3. Moreover, the elementary inequality $x \ln x-(x-1) \leq(x-1)^{2}$ for $x>0$ gives rise to

$$
\begin{gathered}
\overline{n_{\infty}}\left(\frac{\bar{n}}{\overline{n_{\infty}}} \ln \left(\frac{\bar{n}}{\overline{n_{\infty}}}\right)-\left(\frac{\bar{n}}{\overline{n_{\infty}}}-1\right)\right) \leq \overline{n_{\infty}}\left(\frac{\bar{n}}{\overline{n_{\infty}}}-1\right)^{2} \\
\quad \leq 2 \overline{n_{\infty}}\left[\left(\overline{\left(\frac{n}{n_{\infty}}\right)}-1\right)^{2}+\left(\frac{\bar{n}}{\overline{n_{\infty}}}-\overline{\left(\frac{n}{n_{\infty}}\right)}\right)^{2}\right]
\end{gathered}
$$

and an analogous estimate for the corresponding expressions involving $p$. The second term on the right hand side of the previous line can be bounded from above by applying Lemma 3.5, which guarantees a constant $C\left(\gamma, \Gamma, M_{1}\right)>0$ such that

$$
\begin{aligned}
& \left(\frac{\bar{n}}{\overline{n_{\infty}}}-\overline{\left(\frac{n}{n_{\infty}}\right)}\right)^{2} \leq C \int_{\Omega}\left|\nabla \sqrt{\frac{n}{n_{\infty}}}\right|^{2} d x \\
& \quad \leq \frac{C}{4 \inf _{\Omega} n_{\infty}} \int_{\Omega} \frac{1}{n}\left|n_{\infty} \nabla\left(\frac{n}{n_{\infty}}\right)\right|^{2} d x \leq c_{1} \int_{\Omega} \frac{\left|J_{n}\right|^{2}}{n} d x
\end{aligned}
$$

for some constant $c_{1}\left(\gamma, \Gamma, M_{1}\right)>0$. Besides, 


$$
\begin{aligned}
\left(\overline{\left(\frac{n}{n_{\infty}}\right)}-1\right)^{2} & =\frac{1}{n_{*}^{2}}\left(\overline{\left(\frac{n}{\mu_{n}}\right)}-n_{*}\right)^{2}=\frac{1}{n_{*}^{2}}\left(\sqrt{\overline{\left(\frac{n}{\mu_{n}}\right)}}+\sqrt{n_{*}}\right)^{2}\left(\sqrt{\overline{\left(\frac{n}{\mu_{n}}\right)}}-\sqrt{n_{*}}\right)^{2} \\
& =\frac{1}{n_{*}}\left(\sqrt{\overline{\left(\frac{n}{n_{\infty}}\right)}+1}\right)^{2}\left(\sqrt{\overline{\left(\frac{n}{\mu_{n}}\right)}}-\sqrt{n_{*}}\right)^{2} \\
& \leq C\left(\gamma, M_{1}\right)\left(\sqrt{\overline{\left(\frac{n}{\mu_{n}}\right)}}-\sqrt{n_{*}}\right)^{2}
\end{aligned}
$$

See (26) and Lemma 3.4 for the bounds on $n_{*}, n_{\infty}$ and $\bar{n}$. We have thus verified that

$$
\overline{n_{\infty}}\left(\frac{\bar{n}}{\overline{n_{\infty}}} \ln \left(\frac{\bar{n}}{\overline{n_{\infty}}}\right)-\left(\frac{\bar{n}}{\overline{n_{\infty}}}-1\right)\right) \leq c_{2}\left(\int_{\Omega} \frac{\left|J_{n}\right|^{2}}{n} d x+\left(\sqrt{\overline{\left(\frac{n}{\mu_{n}}\right)}}-\sqrt{n_{*}}\right)^{2}\right)
$$

with some $c_{2}\left(\gamma, \Gamma, M_{1}\right)>0$. A similar estimate holds true for the corresponding part of (33) involving $p$.

Considering the last line in (33), we further know that for all $x \in \Omega$ there exists some mean value

$$
\theta(x) \in\left(\min \left\{n_{t r}(x), n_{t r, \infty}\right\}, \max \left\{n_{t r}(x), n_{t r, \infty}\right\}\right)
$$

such that

$$
\int_{n_{t r, \infty}}^{n_{t r}(x)} \ln \left(\frac{s}{1-s}\right) d s=\ln \left(\frac{\theta(x)}{1-\theta(x)}\right)\left(n_{t r}(x)-n_{t r, \infty}\right) .
$$

Consequently,

$$
\varepsilon \int_{\Omega} \int_{n_{t r, \infty}}^{n_{t r}(x)} \ln \left(\frac{s}{1-s}\right) d s d x=\varepsilon \int_{\Omega} \ln \left(\frac{\theta(x)}{1-\theta(x)}\right)\left(n_{t r}(x)-n_{t r, \infty}\right) d x .
$$

In fact, we will prove that there even exists some constant $\xi \in(0,1 / 2)$ such that

$$
\theta(x) \in(\xi, 1-\xi)
$$

for all $x \in \Omega$. Thus, the function $\theta(x)$ is uniformly bounded away from 0 and 1 on $\Omega$. To see this, we first note that $n_{t r, \infty} \in[\gamma, 1-\gamma]$ using the constant $\gamma \in(0,1 / 2)$ from (26). In addition,

$$
\left|\int_{n_{t r, \infty}}^{n_{t r}(x)} \ln \left(\frac{s}{1-s}\right) d s\right| \leq \int_{0}^{1}\left|\ln \left(\frac{s}{1-s}\right)\right| d s=2 \ln (2)
$$

for all $x \in \Omega$. Together with (34), this estimate implies 


$$
\left|\ln \left(\frac{\theta(x)}{1-\theta(x)}\right)\right|\left|n_{t r}(x)-n_{t r, \infty}\right| \leq 2 \ln (2) .
$$

We now choose an arbitrary $x \in \Omega$ and distinguish two cases. If $\left|n_{t r}(x)-n_{t r, \infty}\right| \geq \gamma / 2$, then

$$
\left|\ln \left(\frac{\theta(x)}{1-\theta(x)}\right)\right| \leq \frac{2 \ln (2)}{\left|n_{t r}(x)-n_{t r, \infty}\right|} \leq \frac{4 \ln (2)}{\gamma} .
$$

As a consequence of $\ln (s /(1-s)) \rightarrow \infty$ for $s \rightarrow 1^{-}$and $\ln (s /(1-s)) \rightarrow-\infty$ for $s \rightarrow 0^{+}$, there exists some constant $\xi \in(0, \gamma)$ depending only on $\gamma$ such that $\theta(x) \in(\xi, 1-\xi)$. If $\left|n_{t r}(x)-n_{t r, \infty}\right|<\gamma / 2$, then $n_{t r, \infty} \in[\gamma, 1-\gamma]$ implies $n_{t r}(x) \in(\gamma / 2,1-\gamma / 2)$ and, hence, $\theta(x) \in(\gamma / 2,1-\gamma / 2)$. Again the constant $\xi$ depends only on $\gamma$.

As a result of the calculations above, we may rewrite the last line in (33) as

$$
\begin{aligned}
& \varepsilon \int_{\Omega} \int_{n_{t r, \infty}}^{n_{t r}(x)}\left(\ln \left(\frac{s}{1-s}\right)-\ln \left(\frac{n_{t r, \infty}}{1-n_{t r, \infty}}\right)\right) d s d x \\
& \quad=\varepsilon \int_{\Omega}\left(\ln \left(\frac{\theta(x)}{1-\theta(x)}\right)-\ln \left(\frac{n_{t r, \infty}}{1-n_{t r, \infty}}\right)\right)\left(n_{t r}(x)-n_{t r, \infty}\right) d x .
\end{aligned}
$$

Applying the mean-value theorem to the expression in brackets and observing that

$$
\frac{d}{d s} \ln \left(\frac{s}{1-s}\right)=\frac{1}{s(1-s)},
$$

we find

$$
\begin{gathered}
\varepsilon \int_{\Omega}\left(\ln \left(\frac{\theta(x)}{1-\theta(x)}\right)-\ln \left(\frac{n_{t r, \infty}}{1-n_{t r, \infty}}\right)\right)\left(n_{t r}(x)-n_{t r, \infty}\right) d x \\
=\varepsilon \int_{\Omega} \frac{1}{\sigma(x)(1-\sigma(x))}\left(\theta(x)-n_{t r, \infty}\right)\left(n_{t r}(x)-n_{t r, \infty}\right) d x
\end{gathered}
$$

with some $\sigma(x) \in\left(\min \left\{\theta(x), n_{t r, \infty}\right\}, \max \left\{\theta(x), n_{t r, \infty}\right\}\right)$. Since both $\theta(x), n_{t r, \infty} \in(\xi, 1-\xi)$ for all $x \in \Omega$, we also know that $\sigma(x) \in(\xi, 1-\xi)$ for all $x \in \Omega$. Thus, $(\sigma(x)(1-\sigma(x)))^{-1}$ is bounded uniformly in $\Omega$ in terms of $\xi=\xi(\gamma)$. Consequently,

$$
\begin{aligned}
& \varepsilon \int_{\Omega} \int_{n_{t r, \infty}}^{n_{t r}(x)}\left(\ln \left(\frac{s}{1-s}\right)-\ln \left(\frac{n_{t r, \infty}}{1-n_{t r, \infty}}\right)\right) d s d x \\
& \quad \leq \varepsilon c_{3} \int_{\Omega}\left|\theta(x)-n_{t r, \infty}\right|\left|n_{t r}(x)-n_{t r, \infty}\right| d x \leq \varepsilon c_{3} \int_{\Omega}\left(n_{t r}-n_{t r, \infty}\right)^{2} d x \\
& \quad=\varepsilon c_{3} \int_{\Omega}\left(\sqrt{n_{t r}}+\sqrt{n_{t r, \infty}}\right)^{2}\left(\sqrt{n_{t r}}-\sqrt{n_{t r, \infty}}\right)^{2} d x \leq 4 \varepsilon c_{3} \int_{\Omega}\left(\sqrt{n_{t r}}-\sqrt{n_{t r, \infty}}\right)^{2} d x
\end{aligned}
$$

with a constant $c_{3}(\gamma)>0$ after applying the estimate $\left|\theta(x)-n_{t r, \infty}\right| \leq\left|n_{t r}(x)-n_{t r, \infty}\right|$ for all $x \in \Omega$. Finally, we arrive at 


$$
\begin{aligned}
& E\left(n, p, n_{t r}\right)-E\left(n_{\infty}, p_{\infty}, n_{t r, \infty}\right) \leq C\left(\int_{\Omega}\left(\frac{\left|J_{n}\right|^{2}}{n}+\frac{\left|J_{p}\right|^{2}}{p}\right) d x\right. \\
& \left.\quad+\left(\sqrt{\overline{\left(\frac{n}{\mu_{n}}\right)}}-\sqrt{n_{*}}\right)^{2}+\left(\sqrt{\overline{\left(\frac{p}{\mu_{p}}\right)}}-\sqrt{p_{*}}\right)^{2}+\varepsilon \int_{\Omega}\left(\sqrt{n_{t r}}-\sqrt{n_{t r, \infty}}\right)^{2} d x\right)
\end{aligned}
$$

with a constant $C\left(\gamma, \Gamma, M_{1}\right)>0$.

\section{Abstract versions of the EEP-inequality}

Notation 5.1 We set

$$
n_{t r}^{\prime}:=1-n_{t r}, \quad n_{t r, \infty}^{\prime}:=1-n_{t r, \infty}
$$

and define the positive constants

$$
\begin{aligned}
v_{\infty} & :=\sqrt{\frac{n_{*}}{n_{0}}}=\sqrt{\frac{n_{\infty}}{n_{0} \mu_{n}}}, \quad \pi_{\infty}:=\sqrt{\frac{p_{*}}{p_{0}}}=\sqrt{\frac{p_{\infty}}{p_{0} \mu_{p}}}, \\
v_{t r, \infty} & :=\sqrt{n_{t r, \infty}}, \quad v_{t r, \infty}^{\prime}:=\sqrt{n_{t r, \infty}^{\prime}} .
\end{aligned}
$$

The motivation for introducing the additional variable $n_{t r}^{\prime}$ is the possibility to symmetrise expressions like $\left(n\left(1-n_{t r}\right)-n_{t r}\right)^{2}+\left(p n_{t r}-\left(1-n_{t r}\right)\right)^{2}$ as $\left(n n_{t r}^{\prime}-n_{t r}\right)^{2}+\left(p n_{t r}-n_{t r}^{\prime}\right)^{2}$. Similar terms will appear frequently within the subsequent calculations.

Remark 5.2 We may consider $n_{t r}^{\prime}$ as a fourth independent variable within our model. In this case, the reaction-diffusion system features the following two independent conservation laws:

$$
\begin{aligned}
& \bar{n}-\bar{p}+\varepsilon \overline{n_{t r}}=n_{0} \overline{\mu_{n}\left(\frac{n}{n_{0} \mu_{n}}\right)}-p_{0} \overline{\mu_{p}\left(\frac{p}{p_{0} \mu_{p}}\right)}+\varepsilon \overline{n_{t r}}=M \in \mathbb{R}, \\
& n_{t r}(x)+n_{t r}^{\prime}(x)=1 \text { for all } x \in \Omega .
\end{aligned}
$$

The special formulation of the first conservation law will become clear when looking at the following two Propositions. There, we derive relations for general variables $a, b, c$ and $d$, which correspond to $\sqrt{n /\left(n_{0} \mu_{n}\right)}, \sqrt{p /\left(p_{0} \mu_{p}\right)}, \sqrt{n_{t r}}$ and $\sqrt{n_{t r}^{\prime}}$, respectively.

In addition, we have the following $L^{1}$-bound (cf. Lemma 3.4):

$$
\bar{n}, \bar{p} \leq M_{1} \text {. }
$$


The following Proposition 5.3 establishes an upper bound for the terms in the second line of (32) in the case of constant concentrations $a, b, c$ and $d$. This result is then generalised in Proposition 5.5 to non-constant states $a, b, c, d$.

Proposition 5.3 (Homogeneous concentrations) Let $a, b, c, d \geq 0$ be constants such that their squares satisfy the conservation laws

$$
\begin{aligned}
n_{0} \overline{\mu_{n}} a^{2}-p_{0} \overline{\mu_{p}} b^{2}+\varepsilon c^{2}=M=n_{0} \overline{\mu_{n}} v_{\infty}^{2}-p_{0} \overline{\mu_{p}} \pi_{\infty}^{2}+\varepsilon v_{t r, \infty}^{2}, \\
c^{2}+d^{2}=1=v_{t r, \infty}^{2}+v_{t r, \infty}^{\prime 2}
\end{aligned}
$$

for any $\varepsilon \in\left(0, \varepsilon_{0}\right]$ and arbitrary $\varepsilon_{0}>0$. Moreover, assume

$$
a^{2}, b^{2} \leq C\left(n_{0}, p_{0}, M_{1}, V\right) .
$$

Then, there exists an explicitly computable constant $C\left(\varepsilon_{0}, n_{0}, p_{0}, M, M_{1}, V\right)>0$ such that

$$
\left(a-v_{\infty}\right)^{2}+\left(b-\pi_{\infty}\right)^{2}+\left(c-v_{t r, \infty}\right)^{2} \leq C\left((a d-c)^{2}+(b c-d)^{2}\right)
$$

for all $\varepsilon \in\left(0, \varepsilon_{0}\right]$.

Proof We first introduce the following change of variable: Due to the non-negativity of the concentrations $a, b, c, d$, we define constants $\mu_{1}, \mu_{2}, \mu_{3}, \mu_{4} \in[-1, \infty)$ such that

$$
a=v_{\infty}\left(1+\mu_{1}\right), \quad b=\pi_{\infty}\left(1+\mu_{2}\right), \quad c=v_{t r, \infty}\left(1+\mu_{3}\right), \quad d=v_{t r, \infty}^{\prime}\left(1+\mu_{4}\right),
$$

where $v_{\infty}, \pi_{\infty}, v_{t r, \infty}$ and $v_{t r, \infty}^{\prime}$ are uniformly positive and bounded for all $\varepsilon \in\left(0, \varepsilon_{0}\right]$ in terms of $\varepsilon_{0}, n_{0}, p_{0}, M$ and $V$ by (26). Thus, the boundedness of $a, b, c, d$ implies the existence of a constant $K\left(\varepsilon_{0}, n_{0}, p_{0}, M, M_{1}, V\right)>0$, such that $\mu_{i} \in[-1, K]$ for all $1 \leq i \leq 4$. The left hand side of (35) expressed in terms of the $\mu_{i}$ rewrites as

$$
\left(a-v_{\infty}\right)^{2}+\left(b-\pi_{\infty}\right)^{2}+\left(c-v_{t r, \infty}\right)^{2}=v_{\infty}^{2} \mu_{1}^{2}+\pi_{\infty}^{2} \mu_{2}^{2}+v_{t r, \infty}^{2} \mu_{3}^{2} .
$$

Employing the equilibrium conditions (25), we also find

$$
\begin{aligned}
a d-c & =v_{\infty} v_{t r, \infty}^{\prime}\left(1+\mu_{1}\right)\left(1+\mu_{4}\right)-v_{t r, \infty}\left(1+\mu_{3}\right) \\
& =v_{t r, \infty}\left[\left(1+\mu_{1}\right)\left(1+\mu_{4}\right)-\left(1+\mu_{3}\right)\right]
\end{aligned}
$$

and

$$
\begin{aligned}
b c-d & =\pi_{\infty} v_{t r, \infty}\left(1+\mu_{2}\right)\left(1+\mu_{3}\right)-v_{t r, \infty}^{\prime}\left(1+\mu_{4}\right) \\
& =v_{t r, \infty}^{\prime}\left[\left(1+\mu_{2}\right)\left(1+\mu_{3}\right)-\left(1+\mu_{4}\right)\right] .
\end{aligned}
$$

Moreover, the two conservation laws from the hypotheses rewrite as 


$$
\begin{gathered}
n_{0} \overline{\mu_{n}} v_{\infty}^{2} \mu_{1}\left(2+\mu_{1}\right)-p_{0} \overline{\mu_{p}} \pi_{\infty}^{2} \mu_{2}\left(2+\mu_{2}\right)+\varepsilon v_{t r, \infty}^{2} \mu_{3}\left(2+\mu_{3}\right)=0, \\
v_{t r, \infty}^{2} \mu_{3}\left(2+\mu_{3}\right)+v_{t r, \infty}^{\prime 2} \mu_{4}\left(2+\mu_{4}\right)=0 .
\end{gathered}
$$

The relations (36), (37) allow to express $\varepsilon \mu_{3}$ and $\varepsilon \mu_{4}$ in terms of $\mu_{1}$ and $\mu_{2}$, although not explicitly:

$$
\begin{aligned}
\varepsilon \mu_{3} & =-\frac{n_{0} \overline{\mu_{n}} \nu_{\infty}^{2}}{v_{t r, \infty}^{2}} \frac{2+\mu_{1}}{2+\mu_{3}} \mu_{1}+\frac{p_{0} \overline{\mu_{p}} \pi_{\infty}^{2}}{v_{t r, \infty}^{2}} \frac{2+\mu_{2}}{2+\mu_{3}} \mu_{2} \\
= & :-f_{1,3}\left(\mu_{1}, \mu_{3}\right) \mu_{1}+f_{2,3}\left(\mu_{2}, \mu_{3}\right) \mu_{2}, \\
\varepsilon \mu_{4} & =-\frac{v_{t r, \infty}^{2}}{v_{t r, \infty}^{\prime 2}} \frac{2+\mu_{3}}{2+\mu_{4}} \varepsilon \mu_{3}=:-f_{3,4}\left(\mu_{3}, \mu_{4}\right) \varepsilon \mu_{3} \\
& =: f_{1,4}\left(\mu_{1}, \mu_{3}, \mu_{4}\right) \mu_{1}-f_{2,4}\left(\mu_{2}, \mu_{3}, \mu_{4}\right) \mu_{2},
\end{aligned}
$$

where the last definition follows from inserting the previous expression (38) for $\varepsilon \mu_{3}$ while the factor $2+\mu_{3}$ is bounded in $[1, K+2]$ since $\mu_{i} \in[-1, K]$ for all $1 \leq i \leq 4$. Therefore, all the terms $f_{i, j}$ are uniformly positive as well as bounded from above:

$$
\begin{array}{ll}
0<\underline{C_{1,3}} \leq f_{1,3} \leq \overline{C_{1,3}}<\infty, & 0<\underline{C_{2,3}} \leq f_{2,3} \leq \overline{C_{2,3}}<\infty, \\
0<\underline{C_{3,4}} \leq f_{3,4} \leq \overline{C_{3,4}}<\infty, & 0<\overline{C_{1,4}} \leq f_{1,4} \leq \overline{C_{1,4}}<\infty, \\
0<\underline{C_{2,4}} \leq f_{2,4} \leq \overline{C_{2,4}}<\infty .
\end{array}
$$

All constants $C_{i, j}$ and $\overline{C_{i, j}}$ only depend on $\varepsilon_{0}, n_{0}, p_{0}, M, M_{1}$ and $V$, and there exist corresponding bounds $\underline{C}>0$ and $\bar{C}>0$ such that for all $i, j$

$$
\underline{C} \leq \underline{C_{i, j}}, \overline{C_{i, j}} \leq \bar{C} .
$$

In order to prove (35), we show that under the constraints of the conservation laws (36), (37), respectively, the relations (38), (39), there exists a constant $C\left(\varepsilon_{0}, n_{0}, p_{0}, M, \underline{C}, \bar{C}\right)>0$ for all $\varepsilon \in\left(0, \varepsilon_{0}\right]$ such that

$$
\frac{\left(a-v_{\infty}\right)^{2}+\left(b-\pi_{\infty}\right)^{2}+\left(c-v_{t r, \infty}\right)^{2}}{(a d-c)^{2}+(b c-d)^{2}} \leq C
$$

which is equivalent to

$$
\frac{v_{\infty}^{2} \mu_{1}^{2}+\pi_{\infty}^{2} \mu_{2}^{2}+v_{t r, \infty}^{2} \mu_{3}^{2}}{v_{t r, \infty}^{2}\left[\left(1+\mu_{1}\right)\left(1+\mu_{4}\right)-\left(1+\mu_{3}\right)\right]^{2}+v_{t r, \infty}^{\prime 2}\left[\left(1+\mu_{2}\right)\left(1+\mu_{3}\right)-\left(1+\mu_{4}\right)\right]^{2}} \leq C .
$$

Recall that $v_{\infty}^{2} \leq \Gamma / n_{0}, \pi_{\infty}^{2} \leq \Gamma / p_{0}$ and $v_{t r, \infty}^{2}, v_{t r, \infty}^{\prime 2} \in[\gamma, 1-\gamma]$ with $\gamma \in(0,1 / 2)$ and $\Gamma \in(1 / 2, \infty)$ depending on $\varepsilon_{0}, n_{0}, p_{0}$ and $M$ for all $\varepsilon \in\left(0, \varepsilon_{0}\right]$ (cf. the proof 
of Proposition 26). Since numerator and denominator of (40) are sums of quadratic terms, it is sufficient to bound the denominator from below in terms of its numerator omitting the prefactors $v_{\infty}^{2}, \pi_{\infty}^{2}, v_{t r, \infty}^{2}$ and $v_{t r, \infty}^{\prime 2}$, i.e. to prove that

$$
\begin{aligned}
(*): & :=\left[\left(1+\mu_{1}\right)\left(1+\mu_{4}\right)-\left(1+\mu_{3}\right)\right]^{2}+\left[\left(1+\mu_{2}\right)\left(1+\mu_{3}\right)-\left(1+\mu_{4}\right)\right]^{2} \\
& \geq C\left(\mu_{1}^{2}+\mu_{2}^{2}+\mu_{3}^{2}\right) .
\end{aligned}
$$

More precisely, we will prove that there exists a constant $c\left(\varepsilon_{0}, \underline{C}, \bar{C}\right)>0$ for all $\varepsilon \in\left(0, \varepsilon_{0}\right]$ such that

$$
(*)=\left(\mu_{1}+\mu_{4}+\mu_{1} \mu_{4}-\mu_{3}\right)^{2}+\left(\mu_{2}+\mu_{3}+\mu_{2} \mu_{3}-\mu_{4}\right)^{2} \geq c\left(\mu_{1}^{2}+\mu_{2}^{2}\right)
$$

and that

$$
(*)=\left(\mu_{1}+\mu_{4}+\mu_{1} \mu_{4}-\mu_{3}\right)^{2}+\left(\mu_{2}+\mu_{3}+\mu_{2} \mu_{3}-\mu_{4}\right)^{2} \geq \mu_{3}^{2} .
$$

For this reason, we distinguish four cases and we shall frequently use estimates like

$$
\mu_{i}+\mu_{i} \mu_{j}=\mu_{i}\left(1+\mu_{j}\right) \geq 0 \quad \text { iff } \quad \mu_{i} \geq 0 \quad \text { for all } \quad 1 \leq j \leq 4,
$$

since $\mu_{j} \geq-1$ for all $1 \leq j \leq 4$. We mention already here that all subsequent constants $c_{1}, c_{2}$ are strictly positive and depend only on $\varepsilon_{0}, \underline{C}$ and $\bar{C}$ uniformly for $\varepsilon \in\left(0, \varepsilon_{0}\right]$.

Case 1: $\mu_{1} \geq 0 \wedge \mu_{2} \geq 0$ : If $\mu_{3} \geq 0$, then (37) implies $\mu_{4} \leq 0$ and $\mu_{2}+\mu_{3}+\mu_{2} \mu_{3}-\mu_{4} \geq \mu_{2}$. Moreover, $\mu_{3} \geq 0$ yields

$$
f_{2,3} \mu_{2} \geq f_{1,3} \mu_{1} \Rightarrow \overline{C_{2,3}} \mu_{2} \geq \underline{C_{1,3}} \mu_{1} \Rightarrow \mu_{2} \geq \underline{C_{1,3}} / \overline{C_{2,3}} \mu_{1}
$$

and

$$
\mu_{2}+\mu_{3}+\mu_{2} \mu_{3}-\mu_{4} \geq \mu_{2} \geq \mu_{2} / 2+\underline{C_{1,3}} /\left(2 \overline{C_{2,3}}\right) \mu_{1} \geq c_{1}\left(\mu_{1}+\mu_{2}\right) .
$$

Hence, $\quad(*) \geq\left(\mu_{2}+\mu_{3}+\mu_{2} \mu_{3}-\mu_{4}\right)^{2} \geq c_{2}\left(\mu_{1}^{2}+\mu_{2}^{2}\right) . \quad$ Besides, $(*) \geq\left(\mu_{2}+\mu_{3}+\mu_{2} \mu_{3}-\mu_{4}\right)^{2} \geq \mu_{3}^{2}$. If $\mu_{3}<0$, (37) yields $\mu_{4}>0$ and $\mu_{1}+\mu_{4}+\mu_{1} \mu_{4}-\mu_{3} \geq \mu_{1}$. Since $\mu_{3}<0$, (38) implies

$$
f_{1,3} \mu_{1} \geq f_{2,3} \mu_{2} \Rightarrow \overline{C_{1,3}} \mu_{1} \geq \underline{C_{2,3}} \mu_{2} \Rightarrow \mu_{1} \geq \underline{C_{2,3}} / \overline{C_{1,3}} \mu_{2}
$$

and

$$
\mu_{1}+\mu_{4}+\mu_{1} \mu_{4}-\mu_{3} \geq \mu_{1} \geq \mu_{1} / 2+\underline{C_{2,3}} /\left(2 \overline{C_{1,3}}\right) \mu_{2} \geq c_{1}\left(\mu_{1}+\mu_{2}\right) .
$$

As above, $\quad(*) \geq c_{2}\left(\mu_{1}^{2}+\mu_{2}^{2}\right)$. The signs $\mu_{3} \leq 0 \leq \mu_{1}, \mu_{4}$ yield $(*) \geq\left(\mu_{1}+\mu_{4}+\mu_{1} \mu_{4}-\mu_{3}\right)^{2} \geq \mu_{3}^{2}$. 
Case 2: $\mu_{1} \geq 0 \wedge \mu_{2}<0$ : Equations (38) and (39) imply $\mu_{3} \leq 0$ and $\mu_{4} \geq 0$, and we deduce for all $\varepsilon \in\left(0, \varepsilon_{0}\right]$

$$
\begin{aligned}
\mu_{1} & +\mu_{4}+\mu_{1} \mu_{4}-\mu_{3} \geq \mu_{4}-\mu_{3} \\
& =\varepsilon^{-1}\left(f_{1,3}+f_{1,4}\right) \mu_{1}-\varepsilon^{-1}\left(f_{2,3}+f_{2,4}\right) \mu_{2} \\
& \geq \varepsilon_{0}^{-1}\left(\underline{C_{1,3}}+\underline{C_{1,4}}\right)\left|\mu_{1}\right|+\varepsilon_{0}^{-1}\left(\underline{C_{2,3}}+\underline{C_{2,4}}\right)\left|\mu_{2}\right| \geq c_{1}\left(\left|\mu_{1}\right|+\left|\mu_{2}\right|\right)
\end{aligned}
$$

and, thus, $(*) \geq\left(\mu_{1}+\mu_{4}+\mu_{1} \mu_{4}-\mu_{3}\right)^{2} \geq c_{2}\left(\mu_{1}^{2}+\mu_{2}^{2}\right)$. Since $\mu_{2}, \mu_{3} \leq 0 \leq \mu_{4}$, we have

$$
(*) \geq\left(\mu_{4}-\mu_{3}-\mu_{2}\left(1+\mu_{3}\right)\right)^{2} \geq \mu_{3}^{2} .
$$

Case 3: $\mu_{1}<0 \wedge \mu_{2} \geq 0$ : Here, $\mu_{3} \geq 0$ due to (38) and, thus, $\mu_{4} \leq 0$ by (39), which yields for all $\varepsilon \in\left(0, \varepsilon_{0}\right]$

$$
\begin{gathered}
\mu_{2}+\mu_{3}+\mu_{2} \mu_{3}-\mu_{4} \geq \mu_{3}-\mu_{4}=\varepsilon^{-1}\left(f_{2,3}+f_{2,4}\right) \mu_{2}-\varepsilon^{-1}\left(f_{1,3}+f_{1,4}\right) \mu_{1} \\
\geq \varepsilon_{0}^{-1}\left(\underline{C_{1,3}}+\underline{C_{1,4}}\right)\left|\mu_{1}\right|+\varepsilon_{0}^{-1}\left(\underline{C_{2,3}}+\underline{C_{2,4}}\right)\left|\mu_{2}\right| \geq c_{1}\left(\left|\mu_{1}\right|+\left|\mu_{2}\right|\right)
\end{gathered}
$$

and $(*) \geq\left(\mu_{2}+\mu_{3}+\mu_{2} \mu_{3}-\mu_{4}\right)^{2} \geq c_{2}\left(\mu_{1}^{2}+\mu_{2}^{2}\right)$. And as $\mu_{1}, \mu_{4} \leq 0 \leq \mu_{3}$, one has

$$
(*) \geq\left(\mu_{3}-\mu_{4}-\mu_{1}\left(1+\mu_{4}\right)\right)^{2} \geq \mu_{3}^{2} .
$$

Case 4: $\mu_{1}<0 \wedge \mu_{2}<0$ : Supposing that $\mu_{3} \geq 0$ and thus $\mu_{4} \leq 0$ by (39), we observe

$$
\left|\mu_{1}+\mu_{4}+\mu_{1} \mu_{4}-\mu_{3}\right|=\mu_{3}-\mu_{1}-\mu_{4}\left(1+\mu_{1}\right) \geq-\mu_{1} .
$$

Furthermore, $\mu_{3} \geq 0$ enables us to estimate

$$
f_{1,3} \mu_{1} \leq f_{2,3} \mu_{2} \Rightarrow \overline{C_{1,3}} \mu_{1} \leq \underline{C_{2,3}} \mu_{2} \Rightarrow-\mu_{1} \geq-\underline{C_{2,3}} / \overline{C_{1,3}} \mu_{2} .
$$

and

$$
\left|\mu_{1}+\mu_{4}+\mu_{1} \mu_{4}-\mu_{3}\right| \geq-\mu_{1} \geq-\mu_{1} / 2-\underline{C_{2,3}} /\left(2 \overline{C_{1,3}}\right) \mu_{2} \geq c_{1}\left(\left|\mu_{1}\right|+\left|\mu_{2}\right|\right) .
$$

Hence, $(*) \geq\left(\mu_{1}+\mu_{4}+\mu_{1} \mu_{4}-\mu_{3}\right)^{2} \geq c_{2}\left(\mu_{1}^{2}+\mu_{2}^{2}\right)$. The second estimate in terms of $\mu_{3}^{2}$ follows with $\mu_{1}, \mu_{4} \leq 0 \leq \mu_{3}$ from

$$
(*) \geq\left(\mu_{3}-\mu_{4}-\mu_{1}\left(1+\mu_{4}\right)\right)^{2} \geq \mu_{3}^{2} .
$$

In the opposite case that $\mu_{3}<0$ and thus $\mu_{4} \geq 0$ due to (39), we estimate

$$
\left|\mu_{2}+\mu_{3}+\mu_{2} \mu_{3}-\mu_{4}\right|=\mu_{4}-\mu_{2}-\mu_{3}\left(1+\mu_{2}\right) \geq-\mu_{2}
$$

and 


$$
f_{2,3} \mu_{2} \leq f_{1,3} \mu_{1} \Rightarrow \overline{C_{2,3}} \mu_{2} \leq \underline{C_{1,3}} \mu_{1} \Rightarrow-\mu_{2} \geq-\underline{C_{1,3}} / \overline{C_{2,3}} \mu_{1} .
$$

We, thus, arrive at

$$
\left|\mu_{2}+\mu_{3}+\mu_{2} \mu_{3}-\mu_{4}\right| \geq-\mu_{2} \geq-\mu_{2} / 2-\underline{C_{1,3}} /\left(2 \overline{C_{2,3}}\right) \mu_{1} \geq c_{1}\left(\left|\mu_{1}\right|+\left|\mu_{2}\right|\right)
$$

and $(*) \geq\left(\mu_{2}+\mu_{3}+\mu_{2} \mu_{3}-\mu_{4}\right)^{2} \geq c_{2}\left(\mu_{1}^{2}+\mu_{2}^{2}\right)$. The corresponding inequality for $\mu_{3}$ reads

$$
(*) \geq\left(\mu_{4}-\mu_{3}-\mu_{2}\left(1+\mu_{3}\right)\right)^{2} \geq \mu_{3}^{2},
$$

which follows from $\mu_{2}, \mu_{3} \leq 0 \leq \mu_{4}$.

The proof of the proposition is now complete.

Notation 5.4 From now on, $\|\cdot\|$ without further specification shall always denote the $L^{2}$-norm in $\Omega$.

Within the subsequent Proposition 5.5, the expressions $(a d-c)^{2}$ and $(b c-d)^{2}$ on the right hand side of (35) will be generalised to $\|a d-c\|^{2}$ and $\|b c-d\|^{2}$ in (42). We will later show in the proof of Theorem 1.5 that $\|a d-c\|^{2}$ (and also $\left.\|b c-d\|^{2}\right)$ can be estimated from above via the reaction terms within the entropy production (7) when using the special choices $\sqrt{n /\left(n_{0} \mu_{n}\right)}, \sqrt{p /\left(p_{0} \mu_{p}\right)}, \sqrt{n_{t r}}$, and $\sqrt{n_{t r}^{\prime}}$ for $a, b, c$, and $d$.

Proposition 5.5 (Inhomogeneous concentrations) Let $a, b, c, d: \Omega \rightarrow \mathbb{R}$ be measurable, non-negative functions such that their squares satisfy the conservation laws

$$
\begin{gathered}
n_{0} \overline{\mu_{n} a^{2}}-p_{0} \overline{\mu_{p} b^{2}}+\varepsilon \overline{c^{2}}=M=n_{0} \overline{\mu_{n}} \nu_{\infty}^{2}-p_{0} \overline{\mu_{p}} \pi_{\infty}^{2}+\varepsilon v_{t r, \infty}^{2}, \\
\overline{c^{2}}+\overline{d^{2}}=1=v_{t r, \infty}^{2}+v_{t r, \infty}^{\prime 2}
\end{gathered}
$$

for any $\varepsilon \in\left(0, \varepsilon_{0}\right]$ and arbitrary $\varepsilon_{0}>0$. In addition, we assume

$$
\overline{a^{2}}, \overline{b^{2}} \leq C\left(n_{0}, p_{0}, M_{1}, V\right) .
$$

Then, there exists an explicitly computable constant $C\left(\varepsilon_{0}, n_{0}, p_{0}, M, M_{1}, V\right)>0$ such that

$$
\begin{aligned}
& \left(\sqrt{\overline{a^{2}}}-v_{\infty}\right)^{2}+\left(\sqrt{\overline{b^{2}}}-\pi_{\infty}\right)^{2}+\left\|c-v_{t r, \infty}\right\|^{2} \\
& \leq C\left(\|a d-c\|^{2}+\|b c-d\|^{2}+\|\nabla a\|^{2}+\|\nabla b\|^{2}\right. \\
& \left.\quad+\|a-\bar{a}\|^{2}+\|b-\bar{b}\|^{2}+\|c-\bar{c}\|^{2}+\|d-\bar{d}\|^{2}\right)
\end{aligned}
$$

for all $\varepsilon \in\left(0, \varepsilon_{0}\right]$. 
Proof We divide the proof into two steps. In the first part, we shall derive lower bounds for the reaction terms $\|a d-c\|^{2}+\|b c-d\|^{2}$ involving $(\bar{a} \bar{d}-\bar{c})^{2}+(\bar{b} \bar{c}-\bar{d})^{2}$. This will allow us to apply Proposition 5.3 in the second step.

Step 1: We show

$$
\|a d-c\|^{2} \geq \frac{1}{2}(\bar{a} \bar{d}-\bar{c})^{2}-c_{1}\left(\|a-\bar{a}\|^{2}+\|b-\bar{b}\|^{2}+\|c-\bar{c}\|^{2}+\|d-\bar{d}\|^{2}\right),
$$

and

$$
\|b c-d\|^{2} \geq \frac{1}{2}(\bar{b} \bar{c}-\bar{d})^{2}-c_{1}\left(\|a-\bar{a}\|^{2}+\|b-\bar{b}\|^{2}+\|c-\bar{c}\|^{2}+\|d-\bar{d}\|^{2}\right)
$$

with some explicitly computable constant $c_{1}>0$. For this reason, we define

$$
\delta_{1}:=a-\bar{a}, \quad \delta_{2}:=b-\bar{b}, \quad \delta_{3}:=c-\bar{c}, \quad \delta_{4}:=d-\bar{d}
$$

and note that $\overline{\delta_{1}}=\overline{\delta_{2}}=\overline{\delta_{3}}=\overline{\delta_{4}}=0$. Moreover,

$$
|\bar{a} \bar{d}-\bar{c}|,|\bar{b} \bar{c}-\bar{d}| \leq C\left(n_{0}, p_{0}, M_{1}, V\right)
$$

due to Young's inequality, $\overline{a^{2}}, \overline{b^{2}} \leq C\left(n_{0}, p_{0}, M_{1}, V\right)$ and $\overline{c^{2}}, \overline{d^{2}} \leq 1$.

We now define

$$
S:=\left\{x \in \Omega|| \delta_{1}|\leq 1 \wedge| \delta_{2}|\leq 1 \wedge| \delta_{3}|\leq 1 \wedge| \delta_{4} \mid \leq 1\right\}
$$

and split the squares of the $L^{2}(\Omega)$-norm as

$$
\|a d-c\|^{2}=\int_{S}(a d-c)^{2} d x+\int_{\Omega \backslash S}(a d-c)^{2} d x
$$

and

$$
\|b c-d\|^{2}=\int_{S}(b c-d)^{2} d x+\int_{\Omega \backslash S}(b c-d)^{2} d x,
$$

respectively. In order to estimate the first integral in (43) from below, we write

$$
a d=\left(\bar{a}+\delta_{1}\right)\left(\bar{d}+\delta_{4}\right)=\bar{a} \bar{d}+\bar{a} \delta_{4}+\bar{d} \delta_{1}+\delta_{1} \delta_{4}, \quad c=\bar{c}+\delta_{3} .
$$

This yields 


$$
\begin{aligned}
\int_{S}(a d-c)^{2} d x= & \int_{S}(\bar{a} \bar{d}-\bar{c})^{2} d x+2 \int_{S}(\bar{a} \bar{d}-\bar{c})\left(\bar{a} \delta_{4}+\bar{d} \delta_{1}+\delta_{1} \delta_{4}-\delta_{3}\right) d x \\
& +\int_{S}\left(\bar{a} \delta_{4}+\bar{d} \delta_{1}+\delta_{1} \delta_{4}-\delta_{3}\right)^{2} d x \\
\geq & \frac{1}{2} \int_{S}(\bar{a} \bar{d}-\bar{c})^{2} d x-\int_{S}\left(\bar{a} \delta_{4}+\bar{d} \delta_{1}+\delta_{1} \delta_{4}-\delta_{3}\right)^{2} d x \\
\geq & \frac{1}{2} \int_{S}(\bar{a} \bar{d}-\bar{c})^{2} d x-C\left(n_{0}, p_{0}, M_{1}, V\right)\left(\overline{\delta_{1}^{2}}+\overline{\delta_{3}^{2}}+\overline{\delta_{4}^{2}}\right)
\end{aligned}
$$

where we used Young's inequality $2 x y \geq-x^{2} / 2-2 y^{2}$ for $x, y \in \mathbb{R}$ in the second step and the boundedness of $\delta_{i}, 1 \leq i \leq 4$, in the last step. Similarly, we deduce

$$
\int_{S}(b c-d)^{2} d x \geq \frac{1}{2} \int_{S}(\bar{b} \bar{c}-\bar{d})^{2} d x-C\left(n_{0}, p_{0}, M_{1}, V\right)\left(\overline{\delta_{2}^{2}}+\overline{\delta_{3}^{2}}+\overline{\delta_{4}^{2}}\right) .
$$

The second integral in (43) is mainly estimated by deriving an upper bound for the measure of $\Omega \backslash S$. For all $i \in\{1, \ldots, 4\}$ we have

$$
\left|\left\{\delta_{i}^{2}>1\right\}\right|=\int_{\left\{\delta_{i}^{2}>1\right\}} 1 d x \leq \int_{\Omega} \delta_{i}^{2} d x=\overline{\delta_{i}^{2}}
$$

and, hence,

$$
|\Omega \backslash S| \leq \sum_{i=1}^{4}\left|\left\{\delta_{i}^{2}>1\right\}\right| \leq \overline{\delta_{1}^{2}}+\overline{\delta_{2}^{2}}+\overline{\delta_{3}^{2}}+\overline{\delta_{4}^{2}}
$$

As a consequence of $|\bar{a} \bar{d}-\bar{c}| \leq C\left(n_{0}, p_{0}, M_{1}, V\right)$, we obtain

$$
\begin{aligned}
\int_{\Omega \backslash S}(\bar{a} \bar{d}-\bar{c})^{2} d x & \leq C\left(n_{0}, p_{0}, M_{1}, V\right)|\{\Omega \backslash S\}| \\
& \leq C\left(n_{0}, p_{0}, M_{1}, V\right)\left(\overline{\delta_{1}^{2}}+\overline{\delta_{2}^{2}}+\overline{\delta_{3}^{2}}+\overline{\delta_{4}^{2}}\right) .
\end{aligned}
$$

This implies

$$
\begin{aligned}
\int_{\Omega \backslash S}(a d-c)^{2} d x \geq 0 & \geq \frac{1}{2} \int_{\Omega \backslash S}(\bar{a} \bar{d}-\bar{c})^{2} d x \\
& -C\left(n_{0}, p_{0}, M_{1}, V\right)\left(\overline{\delta_{1}^{2}}+\overline{\delta_{2}^{2}}+\overline{\delta_{3}^{2}}+\overline{\delta_{4}^{2}}\right)
\end{aligned}
$$

and, analogously,

$$
\begin{aligned}
\int_{\Omega \backslash S}(b c-d)^{2} d x \geq 0 \geq & \frac{1}{2} \int_{\Omega \backslash S}(\bar{b} \bar{c}-\bar{d})^{2} d x \\
& -C\left(n_{0}, p_{0}, M_{1}, V\right)\left(\overline{\delta_{1}^{2}}+\overline{\delta_{2}^{2}}+\overline{\delta_{3}^{2}}+\overline{\delta_{4}^{2}}\right) .
\end{aligned}
$$

Taking the sum of both contributions to (43), we finally arrive at 


$$
\|a d-c\|^{2} \geq \frac{1}{2}(\bar{a} \bar{d}-\bar{c})^{2}-c_{1}\left(n_{0}, p_{0}, M_{1}, V\right)\left(\overline{\delta_{1}^{2}}+\overline{\delta_{2}^{2}}+\overline{\delta_{3}^{2}}+\overline{\delta_{4}^{2}}\right)
$$

and

$$
\|b c-d\|^{2} \geq \frac{1}{2}(\bar{b} \bar{c}-\bar{d})^{2}-c_{1}\left(n_{0}, p_{0}, M_{1}, V\right)\left(\overline{\delta_{1}^{2}}+\overline{\delta_{2}^{2}}+\overline{\delta_{3}^{2}}+\overline{\delta_{4}^{2}}\right) .
$$

Step 2: We introduce constants $\mu_{i} \geq-1,1 \leq i \leq 4$, such that

$$
\begin{aligned}
& \overline{a^{2}}=v_{\infty}^{2}\left(1+\mu_{1}\right)^{2}, \quad \overline{b^{2}}=\pi_{\infty}^{2}\left(1+\mu_{2}\right)^{2}, \\
& \overline{c^{2}}=v_{t r, \infty}^{2}\left(1+\mu_{3}\right)^{2}, \quad \overline{d^{2}}=v_{t r, \infty}^{\prime 2}\left(1+\mu_{4}\right)^{2} .
\end{aligned}
$$

We recall that (26) guarantees the uniform positivity and boundedness of $\nu_{\infty}, \pi_{\infty}, v_{t r, \infty}$, and $v_{t r, \infty}^{\prime}$ for all $\varepsilon \in\left(0, \varepsilon_{0}\right]$ in terms of $\varepsilon_{0}, n_{0}, p_{0}, M$, and $V$. Therefore, the bounds $\overline{a^{2}}, \overline{b^{2}} \leq C\left(n_{0}, p_{0}, M_{1}, V\right)$ and $\overline{c^{2}}, \overline{d^{2}} \leq 1$ give rise to a constant $K\left(\varepsilon_{0}, n_{0}, p_{0}, M, M_{1}, V\right)>0$ such that $\mu_{i} \in[-1, K]$ for all $1 \leq i \leq 4$ uniformly for $\varepsilon \in\left(0, \varepsilon_{0}\right]$.

We now want to derive a formula for $\bar{a}$ in terms of $\delta_{1}$ and $\mu_{1}$. Since $\overline{a^{2}}-\bar{a}^{2}=\|a-\bar{a}\|^{2}=\left\|\delta_{1}\right\|^{2}=\overline{\delta_{1}^{2}}$, one finds

$$
\bar{a}=\sqrt{\overline{a^{2}}}-\frac{\overline{\delta_{1}^{2}}}{\sqrt{\overline{a^{2}}}+\bar{a}}=v_{\infty}\left(1+\mu_{1}\right)-\frac{\overline{\delta_{1}^{2}}}{\sqrt{\overline{a^{2}}}+\bar{a}}
$$

and analogous expressions for $\bar{b}, \bar{c}$ and $\bar{d}$ :

$$
\begin{aligned}
& \bar{b}=\pi_{\infty}\left(1+\mu_{2}\right)-\frac{\overline{\delta_{2}^{2}}}{\sqrt{\overline{b^{2}}}+\bar{b}}, \quad \bar{c}=v_{t r, \infty}\left(1+\mu_{3}\right)-\frac{\overline{\delta_{3}^{2}}}{\sqrt{\overline{c^{2}}}+\bar{c}}, \\
& \bar{d}=v_{t r, \infty}^{\prime}\left(1+\mu_{4}\right)-\frac{\overline{\delta_{4}^{2}}}{\sqrt{\overline{d^{2}}}+\bar{d}} \text {. }
\end{aligned}
$$

Furthermore,

$$
\left(\sqrt{\overline{a^{2}}}-v_{\infty}\right)^{2}=v_{\infty}^{2} \mu_{1}^{2}, \quad\left(\sqrt{\overline{b^{2}}}-\pi_{\infty}\right)^{2}=\pi_{\infty}^{2} \mu_{2}^{2}
$$

and, similarly, 


$$
\begin{aligned}
\left\|c-v_{t r, \infty}\right\|^{2} & =\overline{c^{2}}-2 \bar{c} v_{t r, \infty}+v_{t r, \infty}^{2} \\
& =v_{t r, \infty}^{2}\left(1+\mu_{3}\right)^{2}-2 v_{t r, \infty}^{2}\left(1+\mu_{3}\right)+\frac{2 v_{t r, \infty} \overline{\delta_{3}^{2}}}{\sqrt{\overline{c^{2}}}+\bar{c}}+v_{t r, \infty}^{2} \\
& =v_{t r, \infty}^{2} \mu_{3}^{2}+\frac{2 v_{t r, \infty}}{\sqrt{\overline{c^{2}}}+\bar{c}} \overline{\delta_{3}^{2}} .
\end{aligned}
$$

One observes that the expansions above in terms of $\overline{\delta_{i}^{2}}$ are singular if, e.g., $\overline{a^{2}}$ is zero. We therefore distinguish the following two cases.

Case 1: $\overline{a^{2}} \geq \kappa^{2} \wedge \overline{b^{2}} \geq \kappa^{2} \wedge \overline{c^{2}} \geq \kappa^{2} \wedge \overline{d^{2}} \geq \kappa^{2}$ : The constant $\kappa>0$ will be chosen according to the calculations in the other Case 2. Here, we have

$$
\frac{1}{\sqrt{\overline{a^{2}}}+\bar{a}}, \quad \frac{1}{\sqrt{\overline{b^{2}}}+\bar{b}}, \quad \frac{1}{\sqrt{\overline{c^{2}}}+\bar{c}}, \quad \frac{1}{\sqrt{\overline{d^{2}}}+\bar{d}} \leq \frac{1}{\kappa}
$$

and

$$
\frac{v_{t r, \infty}^{\prime}}{\sqrt{\overline{a^{2}}}+\bar{a}}, \quad \frac{v_{t r, \infty}}{\sqrt{\overline{b^{2}}}+\bar{b}}, \quad \frac{\pi_{\infty}}{\sqrt{\overline{c^{2}}}+\bar{c}}, \quad \frac{v_{\infty}}{\sqrt{\overline{d^{2}}}+\bar{d}} \leq C\left(\kappa, \varepsilon_{0}, n_{0}, p_{0}, M, V\right)
$$

for all $\varepsilon \in\left(0, \varepsilon_{0}\right]$ due to the bounds on $v_{\infty}$ and $\pi_{\infty}$ from (26). Equation (46) further implies

$$
\begin{aligned}
(\bar{a} \bar{d}-\bar{c})^{2}= & \left(v_{\infty} v_{t r, \infty}^{\prime}\left(1+\mu_{1}\right)\left(1+\mu_{4}\right)-\frac{v_{\infty}\left(1+\mu_{1}\right)}{\sqrt{\overline{d^{2}}}+\bar{d}} \overline{\delta_{4}^{2}}-\frac{v_{t r, \infty}^{\prime}\left(1+\mu_{4}\right)}{\sqrt{\overline{a^{2}}}+\bar{a}} \delta_{1}^{2}\right. \\
& \left.+\frac{1}{\left(\sqrt{\overline{a^{2}}}+\bar{a}\right)\left(\sqrt{\overline{d^{2}}}+\bar{d}\right)} \overline{\delta_{1}^{2}} \frac{\overline{\delta_{3}^{2}}}{\sqrt{\bar{c}_{4}^{2}}+\bar{c}}\right)^{2} \\
\geq & v_{t r, \infty}^{2}\left(\left(1+\mu_{1}\right)\left(1+\mu_{4}\right)-\left(1+\mu_{3}\right)\right)^{2} \\
& -c_{2}\left(\kappa, \varepsilon_{0}, n_{0}, p_{0}, M, M_{1}, V\right)\left(\overline{\delta_{1}^{2}}+\overline{\delta_{3}^{2}}+\overline{\delta_{4}^{2}}\right)
\end{aligned}
$$

with_an explicit constant $c_{2}$ thanks to $v_{\infty} v_{t r, \infty}^{\prime}=v_{t r, \infty}$ (cf. (25)) and $\left|\mu_{i}\right|, \bar{\delta}_{i}^{2} \leq c_{1}\left(\varepsilon_{0}, n_{0}, p_{0}, M, M_{1}, V\right)$. In a similar fashion using $\pi_{\infty} v_{t r, \infty}=v_{t r, \infty}^{\prime}$, one obtains 


$$
\begin{aligned}
(\bar{b} \bar{c}-\bar{d})^{2}= & \left(\pi_{\infty} v_{t r, \infty}\left(1+\mu_{2}\right)\left(1+\mu_{3}\right)-\frac{\pi_{\infty}\left(1+\mu_{2}\right)}{\sqrt{\overline{c^{2}}}+\bar{c}} \overline{\delta_{3}^{2}}-\frac{v_{t r, \infty}\left(1+\mu_{3}\right)}{\sqrt{\overline{b^{2}}}+\bar{b}} \overline{\delta_{2}^{2}}\right. \\
& \left.+\frac{1}{\left(\sqrt{\overline{b^{2}}}+\bar{b}\right)\left(\sqrt{\overline{c^{2}}}+\bar{c}\right)} \overline{\delta_{2}^{2}} \frac{\overline{\delta_{4}^{2}}}{\delta_{3}^{2}}-v_{t r, \infty}^{\prime}\left(1+\mu_{4}\right)+\frac{\left(\sqrt{\overline{d^{2}}}+\bar{d}\right.}{{ }^{2}}\right) \\
\geq & v_{t r, \infty}^{\prime 2}\left(\left(1+\mu_{2}\right)\left(1+\mu_{3}\right)-\left(1+\mu_{4}\right)\right)^{2} \\
& -c_{2}\left(\kappa, \varepsilon_{0}, n_{0}, p_{0}, M, M_{1}, V\right)\left(\overline{\delta_{2}^{2}}+\overline{\delta_{3}^{2}}+\overline{\delta_{4}^{2}}\right) .
\end{aligned}
$$

In order to finish the proof, it is-according to Step 1-sufficient to show that

$$
\begin{aligned}
& v_{\infty}^{2} \mu_{1}^{2}+\pi_{\infty}^{2} \mu_{2}^{2}+v_{t r, \infty}^{2} \mu_{3}^{2}+\frac{2 v_{t r, \infty}}{\sqrt{\overline{c^{2}}}+\bar{c}} \overline{\delta_{3}^{2}} \leq C_{1}\left(\|\nabla a\|^{2}+\|\nabla b\|^{2}\right. \\
& \left.+\frac{1}{2}(\bar{a} \bar{d}-\bar{c})^{2}+\frac{1}{2}(\bar{b} \bar{c}-\bar{d})^{2}-2 c_{1}\left(n_{0}, p_{0}, M_{1}, V\right)\left(\overline{\delta_{1}^{2}}+\overline{\delta_{2}^{2}}+\overline{\delta_{3}^{2}}+\overline{\delta_{4}^{2}}\right)\right) \\
& +C_{2}\left(\overline{\delta_{1}^{2}}+\overline{\delta_{2}^{2}}+\overline{\delta_{3}^{2}}+\overline{\delta_{4}^{2}}\right)
\end{aligned}
$$

for appropriate constants $C_{1}, C_{2}>0$. But due to Step 2 it is sufficient to show that for suitable constants $C_{1}, C_{2}>0$,

$$
\begin{aligned}
& v_{\infty}^{2} \mu_{1}^{2}+\pi_{\infty}^{2} \mu_{2}^{2}+v_{t r, \infty}^{2} \mu_{3}^{2}+\frac{2 v_{t r, \infty}}{\sqrt{\overline{c^{2}}}+\bar{c}} \overline{\delta_{3}^{2}} \leq C_{1}\left(\|\nabla a\|^{2}+\|\nabla b\|^{2}\right. \\
& +\frac{v_{t r, \infty}^{2}}{2}\left(\left(1+\mu_{1}\right)\left(1+\mu_{4}\right)-\left(1+\mu_{3}\right)\right)^{2}+\frac{v_{t r, \infty}^{\prime 2}}{2}\left(\left(1+\mu_{2}\right)\left(1+\mu_{3}\right)-\left(1+\mu_{4}\right)\right)^{2} \\
& \left.-c_{3}\left(\kappa, \varepsilon_{0}, n_{0}, p_{0}, M, M_{1}, V\right)\left(\overline{\delta_{1}^{2}}+\overline{\delta_{2}^{2}}+\overline{\delta_{3}^{2}}+\overline{\delta_{4}^{2}}\right)\right)+C_{2}\left(\overline{\delta_{1}^{2}}+\overline{\delta_{2}^{2}}+\overline{\delta_{3}^{2}}+\overline{\delta_{4}^{2}}\right) .
\end{aligned}
$$

Collecting all $\overline{\delta_{i}^{2}}$-terms on the right hand side, one only has to prove that

$$
\begin{aligned}
& v_{\infty}^{2} \mu_{1}^{2}+\pi_{\infty}^{2} \mu_{2}^{2}+v_{t r, \infty}^{2} \mu_{3}^{2} \leq C_{1}\left(\|\nabla a\|^{2}+\|\nabla b\|^{2}\right. \\
& \left.+v_{t r, \infty}^{2}\left(\left(1+\mu_{1}\right)\left(1+\mu_{4}\right)-\left(1+\mu_{3}\right)\right)^{2}+v_{t r, \infty}^{\prime 2}\left(\left(1+\mu_{2}\right)\left(1+\mu_{3}\right)-\left(1+\mu_{4}\right)\right)^{2}\right) \\
& \quad+\left(C_{2}-C\left(C_{1}, \kappa, \varepsilon_{0}, n_{0}, p_{0}, M, M_{1}, V\right)\right)\left(\overline{\delta_{1}^{2}}+\overline{\delta_{2}^{2}}+\overline{\delta_{3}^{2}}+\overline{\delta_{4}^{2}}\right)
\end{aligned}
$$

or, equivalently, 


$$
\begin{aligned}
& \left(\sqrt{\overline{a^{2}}}-v_{\infty}\right)^{2}+\left(\sqrt{\overline{b^{2}}}-\pi_{\infty}\right)^{2}+\left(\sqrt{\overline{c^{2}}}-v_{t r, \infty}\right)^{2} \\
& \leq C_{1}\left(\left(\sqrt{\overline{a^{2}}} \sqrt{\overline{d^{2}}}-\sqrt{\overline{c^{2}}}\right)^{2}+\left(\sqrt{\overline{b^{2}}} \sqrt{\overline{c^{2}}}-\sqrt{\overline{d^{2}}}\right)^{2}+\|\nabla a\|^{2}+\|\nabla b\|^{2}\right) \\
& \quad+\left(C_{2}-C\left(C_{1}, \kappa, \varepsilon_{0}, n_{0}, p_{0}, M, M_{1}, V\right)\right)\left(\overline{\delta_{1}^{2}}+\overline{\delta_{2}^{2}}+\overline{\delta_{3}^{2}}+\overline{\delta_{4}^{2}}\right) .
\end{aligned}
$$

In order to verify (47), we start with the estimate

$$
\left(\sqrt{\overline{a^{2}}}-v_{\infty}\right)^{2} \leq 2\left[\left(\sqrt{\frac{\overline{\mu_{n} a^{2}}}{\overline{\mu_{n}}}}-v_{\infty}\right)^{2}+\left(\sqrt{\frac{\overline{\mu_{n} a^{2}}}{\overline{\mu_{n}}}}-\sqrt{\overline{a^{2}}}\right)^{2}\right]
$$

and a corresponding one involving $b$. The last term on the right hand side satisfies

$$
\begin{aligned}
& \left(\sqrt{\frac{\overline{\mu_{n} a^{2}}}{\overline{\mu_{n}}}}-\sqrt{\overline{a^{2}}}\right)^{2}=\frac{\left(\overline{\frac{\mu_{n} a^{2}}{\overline{\mu_{n}}}}-\overline{a^{2}}\right)^{2}}{\left(\sqrt{\frac{\overline{\mu_{n} a^{2}}}{\overline{\mu_{n}}}}+\sqrt{\overline{a^{2}}}\right)^{2}} \leq \frac{1}{\kappa^{2}}\left(\frac{\overline{\mu_{n} a^{2}}}{\overline{\mu_{n}}}-\overline{a^{2}}\right)^{2} \\
& \leq c \int_{\Omega}\left|\nabla \sqrt{a^{2}}\right|^{2} d x=c\|\nabla a\|^{2}
\end{aligned}
$$

due to Lemma 3.5 with a constant $c\left(\kappa, n_{0}, p_{0}, M_{1}, V\right)>0$. Similarly,

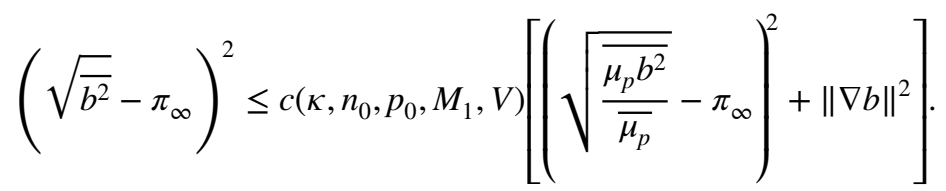

Proposition 5.3 (with $a^{2}, b^{2}, c^{2}$, and $d^{2}$ therein replaced by $\overline{\mu_{n} a^{2}} / \overline{\mu_{n}}, \overline{\mu_{p} b^{2}} / \overline{\mu_{p}}$, $\overline{c^{2}}$, and $\overline{d^{2}}$ ) tells us that there exists an explicitly computable constant $C\left(\varepsilon_{0}, n_{0}, p_{0}, M, M_{1}, V\right)>0$ such that

$$
\begin{aligned}
& \left(\sqrt{\frac{\overline{\mu_{n} a^{2}}}{\overline{\mu_{n}}}}-v_{\infty}\right)^{2}+\left(\sqrt{\frac{\overline{\mu_{p} b^{2}}}{\overline{\mu_{p}}}}-\pi_{\infty}\right)^{2}+\left(\sqrt{\overline{c^{2}}}-v_{t r, \infty}\right)^{2} \\
& \left.\leq C\left(\sqrt{\frac{\overline{\mu_{n} a^{2}}}{\overline{\mu_{n}}}} \sqrt{\overline{d^{2}}}-\sqrt{\overline{c^{2}}}\right)^{2}+\left(\sqrt{\frac{\overline{\mu_{p} b^{2}}}{\overline{\mu_{p}}}} \sqrt{\overline{c^{2}}}-\sqrt{\overline{d^{2}}}\right)^{2}\right)
\end{aligned}
$$


for all $\varepsilon \in\left(0, \varepsilon_{0}\right]$. Using an analogue expansion as before, we further deduce with $\overline{d^{2}} \leq 1$,

$$
\begin{aligned}
\left(\sqrt{\frac{\overline{\mu_{n} a^{2}}}{\overline{\mu_{n}}}} \sqrt{\overline{d^{2}}}-\sqrt{\overline{c^{2}}}\right)^{2} & =\left(\sqrt{\overline{a^{2}}} \sqrt{\overline{d^{2}}}-\sqrt{\overline{c^{2}}}+\left(\sqrt{\frac{\overline{\mu_{n} a^{2}}}{\overline{\mu_{n}}}}-\sqrt{\overline{\overline{a^{2}}}}\right) \sqrt{\overline{\overline{d^{2}}}}\right)^{2} \\
& \leq c\left(\kappa, n_{0}, p_{0}, M_{1}, V\right)\left(\left(\sqrt{\overline{a^{2}}} \sqrt{\overline{d^{2}}}-\sqrt{\overline{\bar{c}}}\right)^{2}+\|\nabla a\|^{2}\right)
\end{aligned}
$$

As a corresponding estimate holds true also for the other expression on the right hand side of (48), we have shown that there exists a constant $C_{1}\left(\kappa, \varepsilon_{0}, n_{0}, p_{0}, M, M_{1}, V\right)>0$ independent of $\varepsilon$ for $\varepsilon \in\left(0, \varepsilon_{0}\right]$ such that

$$
\begin{aligned}
& \left(\sqrt{\overline{a^{2}}}-v_{\infty}\right)^{2}+\left(\sqrt{\overline{b^{2}}}-\pi_{\infty}\right)^{2}+\left(\sqrt{\overline{c^{2}}}-v_{t r, \infty}\right)^{2} \\
& \leq C_{1}\left(\left(\sqrt{\overline{a^{2}}} \sqrt{\overline{d^{2}}}-\sqrt{\overline{c^{2}}}\right)^{2}+\left(\sqrt{\overline{b^{2}}} \sqrt{\overline{c^{2}}}-\sqrt{\overline{d^{2}}}\right)^{2}+\|\nabla a\|^{2}+\|\nabla b\|^{2}\right) .
\end{aligned}
$$

Choosing $C_{2}>0$ now sufficiently large, Eq. (47) holds true.

Case 2: $\overline{a^{2}}<\kappa^{2} \vee \overline{b^{2}}<\kappa^{2} \vee \overline{c^{2}}<\kappa^{2} \vee \overline{d^{2}}<\kappa^{2}$ : In this case, we will not need Proposition 5.3 and we shall directly prove (42) employing only the result of Step 1. In fact, for $\kappa$ chosen sufficiently small, the states considered in Case 2 are necessarily bounded away from the equilibrium and the following arguments show that consequentially the right hand side of (42) is also bounded away from zero, which allows to close the estimate (42). As a result of the hypotheses $\overline{a^{2}}, \overline{b^{2}} \leq C\left(n_{0}, p_{0}, M_{1}, V\right)$ and $\overline{c^{2}}, \overline{d^{2}} \leq 1$, we use Young's inequality to estimate $\bar{a}, \bar{b}, \bar{c}, \bar{d} \leq c\left(n_{0}, p_{0}, M_{1}, V\right)$ and

$$
\left(\sqrt{\overline{a^{2}}}-v_{\infty}\right)^{2}+\left(\sqrt{\overline{b^{2}}}-\pi_{\infty}\right)^{2}+\left\|c-v_{t r, \infty}\right\|^{2} \leq C\left(\varepsilon_{0}, n_{0}, p_{0}, M, M_{1}, V\right)
$$

with $C>0$ uniformly for $\varepsilon \in\left(0, \varepsilon_{0}\right]$. We stress that the subsequent cases are not necessarily exclusive.

Case 2.1: $\overline{c^{2}}<\kappa^{2}$ : First, $\bar{c}=\sqrt{\bar{c}^{2}} \leq \sqrt{\overline{c^{2}}}<\kappa$. This yields

$$
\begin{aligned}
\overline{d^{2}} & =1-\overline{c^{2}}>1-\kappa^{2} \Rightarrow \bar{d}^{2}=\overline{d^{2}}-\overline{\delta_{4}^{2}}>1-\overline{\delta_{4}^{2}}-\kappa^{2} \\
& \Rightarrow(\bar{b} \bar{c}-\bar{d})^{2} \geq \bar{d}^{2}-2 \bar{b} \bar{c} \bar{d}>1-\overline{\delta_{4}^{2}}-\kappa^{2}-2 \bar{b} \bar{d} \kappa \\
& \geq 1-\overline{\delta_{4}^{2}}-\kappa^{2}-C\left(n_{0}, p_{0}, M_{1}, V\right) \kappa
\end{aligned}
$$

For $\kappa>0$ sufficiently small, we then have $0<1-C\left(n_{0}, p_{0}, M_{1}, V\right) \kappa-\kappa^{2}$ $\leq(\bar{b} \bar{c}-\bar{d})^{2}+\overline{\delta_{4}^{2}}$ and, hence, 


$$
\begin{aligned}
& \left(\sqrt{\overline{a^{2}}}-v_{\infty}\right)^{2}+\left(\sqrt{\overline{b^{2}}}-\pi_{\infty}\right)^{2}+\left\|c-v_{t r, \infty}\right\|^{2} \\
& \leq C\left(\varepsilon_{0}, n_{0}, p_{0}, M, M_{1}, V\right) \leq K(\bar{b} \bar{c}-\bar{d})^{2}+K \overline{\delta_{4}^{2}} \\
& \leq 2 K\|b c-d\|^{2}+\left(2 K c_{1}\left(n_{0}, p_{0}, M_{1}, V\right)+K\right)\left(\overline{\delta_{1}^{2}}+\overline{\delta_{2}^{2}}+\overline{\delta_{3}^{2}}+\overline{\delta_{4}^{2}}\right)
\end{aligned}
$$

by (45) with some $K\left(\kappa, \varepsilon_{0}, n_{0}, p_{0}, M, M_{1}, V\right)>0$. Let us call the parameter $\kappa$ from

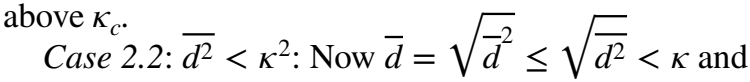

$$
\begin{aligned}
\overline{c^{2}} & =1-\overline{d^{2}}>1-\kappa^{2} \Rightarrow \bar{c}^{2}=\overline{c^{2}}-\overline{\delta_{3}^{2}}>1-\overline{\delta_{3}^{2}}-\kappa^{2} \\
& \Rightarrow(\bar{a} \bar{d}-\bar{c})^{2} \geq \bar{c}^{2}-2 \bar{a} \bar{c} \bar{d}>1-\overline{\delta_{3}^{2}}-\kappa^{2}-2 \bar{a} \bar{c} \kappa \\
& \geq 1-\overline{\delta_{3}^{2}}-\kappa^{2}-C\left(n_{0}, p_{0}, M_{1}, V\right) \kappa .
\end{aligned}
$$

Again $\kappa>0$ sufficiently small gives rise to $0<1-C\left(n_{0}, p_{0}, M_{1}, V\right) \kappa-\kappa^{2}$ $\leq(\bar{a} \bar{d}-\bar{c})^{2}+\delta_{3}^{2}$ and

$$
\begin{aligned}
& \left(\sqrt{\overline{a^{2}}}-v_{\infty}\right)^{2}+\left(\sqrt{\overline{b^{2}}}-\pi_{\infty}\right)^{2}+\left\|c-v_{t r, \infty}\right\|^{2} \\
& \quad \leq C\left(\varepsilon_{0}, n_{0}, p_{0}, M, M_{1}, V\right) \leq K(\bar{a} \bar{d}-\bar{c})^{2}+K \overline{\delta_{3}^{2}} \\
& \quad \leq 2 K\|a d-c\|^{2}+\left(2 K c_{1}\left(n_{0}, p_{0}, M_{1}, V\right)+K\right)\left(\overline{\delta_{1}^{2}}+\overline{\delta_{2}^{2}}+\overline{\delta_{3}^{2}}+\overline{\delta_{4}^{2}}\right)
\end{aligned}
$$

for some constant $K\left(\kappa, \varepsilon_{0}, n_{0}, p_{0}, M, M_{1}, V\right)>0$ using (44). This $\kappa>0$ shall be denoted by $\kappa_{d}$.

Case 2.3: $\overline{a^{2}}<\kappa^{2}$ : We first notice that $\bar{a}<\kappa$ and $2 \bar{c} \bar{d} \leq \bar{c}^{2}+\bar{d}^{2} \leq \overline{c^{2}}+\overline{d^{2}}=1$. Now, we choose $\kappa_{a}:=\kappa>0$ sufficiently small such that $2 \kappa<\kappa_{c}^{2}$. Then, if $\overline{c^{2}}<2 \kappa$, we have $\overline{c^{2}}<\kappa_{c}^{2}$, and the estimate

$$
\begin{aligned}
& \left(\sqrt{\overline{a^{2}}}-v_{\infty}\right)^{2}+\left(\sqrt{\overline{b^{2}}}-\pi_{\infty}\right)^{2}+\left\|c-v_{t r, \infty}\right\|^{2} \\
& \quad \leq 2 K\|b c-d\|^{2}+\left(2 K c_{1}\left(n_{0}, p_{0}, M_{1}, V\right)+K\right)\left(\overline{\delta_{1}^{2}}+\overline{\delta_{2}^{2}}+\overline{\delta_{3}^{2}}+\overline{\delta_{4}^{2}}\right)
\end{aligned}
$$

with $K\left(\kappa, \varepsilon_{0}, n_{0}, p_{0}, M, M_{1}, V\right)>0$ immediately follows from Case 2.1. And if $\overline{c^{2}} \geq 2 \kappa$, then

$$
\bar{c}^{2}=\overline{c^{2}}-\overline{\delta_{3}^{2}} \geq 2 \kappa-\overline{\delta_{3}^{2}} \Rightarrow(\bar{a} \bar{d}-\bar{c})^{2} \geq \bar{c}^{2}-2 \bar{a} \bar{c} \bar{d} \geq 2 \kappa-\overline{\delta_{3}^{2}}-\kappa=\kappa-\overline{\delta_{3}^{2}} .
$$

Consequently, $0<\kappa \leq(\bar{a} \bar{d}-\bar{c})^{2}+\overline{\delta_{3}^{2}}$ and 


$$
\begin{aligned}
& \left(\sqrt{\overline{a^{2}}}-v_{\infty}\right)^{2}+\left(\sqrt{\overline{b^{2}}}-\pi_{\infty}\right)^{2}+\left\|c-v_{t r, \infty}\right\|^{2} \\
& \leq C\left(\varepsilon_{0}, n_{0}, p_{0}, M, M_{1}, V\right) \leq K(\bar{a} \bar{d}-\bar{c})^{2}+K \overline{\delta_{3}^{2}} \\
& \leq 2 K\|a d-c\|^{2}+\left(2 K c_{1}\left(n_{0}, p_{0}, M_{1}, V\right)+K\right)\left(\overline{\delta_{1}^{2}}+\overline{\delta_{2}^{2}}+\overline{\delta_{3}^{2}}+\overline{\delta_{4}^{2}}\right)
\end{aligned}
$$

due to (44) with a constant $K\left(\kappa, \varepsilon_{0}, n_{0}, p_{0}, M, M_{1}, V\right)>0$.

Case 2.4: $\overline{b^{2}}<\kappa^{2}$ : Again $\bar{b}<\kappa$ and $2 \bar{c} \bar{d} \leq \bar{c}^{2}+\bar{d}^{2} \leq \overline{c^{2}}+\bar{d}^{2}=1$. Here, we choose $\kappa_{b}:=\kappa>0$ sufficiently small such that $2 \kappa<\kappa_{d}^{2}$. If $\overline{d^{2}}<2 \kappa$, we have $\overline{d^{2}}<\kappa_{d}^{2}$, and due to Case 2.2 there exists some $K\left(\kappa, \varepsilon_{0}, n_{0}, p_{0}, M, M_{1}, V\right)>0$ such that

$$
\begin{aligned}
& \left(\sqrt{\overline{a^{2}}}-v_{\infty}\right)^{2}+\left(\sqrt{\overline{b^{2}}}-\pi_{\infty}\right)^{2}+\left\|c-v_{t r, \infty}\right\|^{2} \\
& \leq 2 K\|a d-c\|^{2}+\left(2 K c_{1}\left(n_{0}, p_{0}, M_{1}, V\right)+K\right)\left(\overline{\delta_{1}^{2}}+\overline{\delta_{2}^{2}}+\overline{\delta_{3}^{2}}+\overline{\delta_{4}^{2}}\right)
\end{aligned}
$$

If $\overline{d^{2}} \geq 2 \kappa$, then

$$
\bar{d}^{2}=\overline{d^{2}}-\overline{\delta_{4}^{2}} \geq 2 \kappa-\overline{\delta_{4}^{2}} \Rightarrow(\bar{b} \bar{c}-\bar{d})^{2} \geq \bar{d}^{2}-2 \bar{b} \bar{c} \bar{d} \geq 2 \kappa-\overline{\delta_{4}^{2}}-\kappa=\kappa-\overline{\delta_{4}^{2}} .
$$

This implies $0<\kappa \leq(\bar{b} \bar{c}-\bar{d})^{2}+\overline{\delta_{4}^{2}}$ and

$$
\begin{aligned}
& \left(\sqrt{\overline{a^{2}}}-v_{\infty}\right)^{2}+\left(\sqrt{\overline{b^{2}}}-\pi_{\infty}\right)^{2}+\left\|c-v_{t r, \infty}\right\|^{2} \\
& \leq C\left(\varepsilon_{0}, n_{0}, p_{0}, M, M_{1}, V\right) \leq K(\bar{b} \bar{c}-\bar{d})^{2}+K \overline{\delta_{4}^{2}} \\
& \leq 2 K\|b c-d\|^{2}+\left(2 K c_{1}\left(n_{0}, p_{0}, M_{1}, V\right)+K\right)\left(\overline{\delta_{1}^{2}}+\overline{\delta_{2}^{2}}+\overline{\delta_{3}^{2}}+\overline{\delta_{4}^{2}}\right)
\end{aligned}
$$

with $K\left(\kappa, \varepsilon_{0}, n_{0}, p_{0}, M, M_{1}, V\right)>0$ employing (45).

All arguments within Step 2 remain valid, if we finally set $\kappa:=\min \left(\kappa_{a}, \kappa_{b}, \kappa_{c}, \kappa_{d}\right)$. We also observe that the constants $K>0$ above are independent of $\varepsilon \in\left(0, \varepsilon_{0}\right]$. And since $\kappa$ only depends on $n_{0}, p_{0}, M_{1}$ and $V$, we may skip the explicit dependence of $C_{2}$ on $\kappa$ at the end of Case 1. This finishes the proof.

We already pointed out that $\|a d-c\|^{2}$ and $\|b c-d\|^{2}$ can be controlled by the reaction terms of the entropy production, if we replace $a, b, c, d$ by $\sqrt{n /\left(n_{0} \mu_{n}\right)}$, $\sqrt{p /\left(p_{0} \mu_{p}\right)}, \sqrt{n_{t r}}$ and $\sqrt{n_{t r}^{\prime}}$ (see the proof of Theorem 1.5 in Sect. 6 for details). In this proof, also $\|\nabla a\|^{2},\|\nabla b\|^{2},\|a-\bar{a}\|^{2}$ and $\|b-\bar{b}\|^{2}$ may be bounded by the entropy production. However, $\|c-\bar{c}\|^{2}$ and $\|d-\bar{d}\|^{2}$ may not be estimated with the help of Poincaré's inequality since this would yield terms involving $\nabla n_{t r}$, which do not appear in the entropy production. 
Instead, we are able to derive the following estimates for $\|c-\bar{c}\|^{2}$ and $\|d-\bar{d}\|^{2}$, which describe an indirect diffusion transfer from $c$ to $b$ and from $d$ to $a$, respectively: Even if $c$ and $d$ are lacking an explicit diffusion term in the dynamical equations, they do experience indirect diffusive effects thanks to the reversible reaction dynamics and the diffusivity of $a$ and $b$. This is the interpretation of the following functional inequalities.

Proposition 5.6 (Indirect diffusion transfer) Let $a, b, c, d: \Omega \rightarrow \mathbb{R}$ be non-negative functions such that

$$
c^{2}+d^{2}=1
$$

holds true a.e. in $\Omega$. Then,

$$
\|c-\bar{c}\|^{2} \leq 4\left(\|b c-d\|^{2}+\|b-\bar{b}\|^{2}\right) \quad \text { and } \quad\|d-\bar{d}\|^{2} \leq 4\left(\|a d-c\|^{2}+\|a-\bar{a}\|^{2}\right) .
$$

Proof We only verify the second inequality; the first one can be checked along the same lines. First, we notice that

$$
\|\bar{a} d-c\|=\|a d-c+(\bar{a}-a) d\| \leq\|a d-c\|+\|a-\bar{a}\|
$$

because of the bound $0 \leq d \leq 1$. Besides, we deduce

$$
\left\|\bar{a}^{2} d^{2}-c^{2}\right\|=\|(\bar{a} d+c)(\bar{a} d-c)\| \leq(1+\bar{a})\|\bar{a} d-c\|
$$

employing $0 \leq c, d \leq 1$. For the subsequent estimates, we need two auxiliary inequalities: For every function $f: \Omega \rightarrow \mathbb{R}$ and all $\lambda \in \mathbb{R}$, we have

$$
\begin{aligned}
\|f-\bar{f}\|^{2} & =\int_{\Omega}(f-\lambda+\lambda-\bar{f})^{2} d x \\
& =\int_{\Omega}\left((f-\lambda)^{2}-2(f-\lambda)(\bar{f}-\lambda)+(\bar{f}-\lambda)^{2}\right) d x \\
& =\int_{\Omega}(f-\lambda)^{2} d x-(\bar{f}-\lambda)^{2} \leq\|f-\lambda\|^{2} .
\end{aligned}
$$

And for all $x \geq 0$, one has

$$
\frac{1+x}{\sqrt{1+x^{2}}}=\frac{\sqrt{1+2 x+x^{2}}}{\sqrt{1+x^{2}}} \leq \frac{\sqrt{2\left(1+x^{2}\right)}}{\sqrt{1+x^{2}}}=\sqrt{2} .
$$

Since $c^{2}+d^{2}=1$, we obtain

$$
\begin{aligned}
\left\|\bar{a}^{2} d^{2}-c^{2}\right\| & =\left\|\bar{a}^{2} d^{2}+d^{2}-1\right\|=\left\|\left(1+\bar{a}^{2}\right) d^{2}-1\right\| \\
& =\left\|\left(\sqrt{1+\bar{a}^{2}} d+1\right)\left(\sqrt{1+\bar{a}^{2}} d-1\right)\right\| \\
& \geq\left\|\sqrt{1+\bar{a}^{2}} d-1\right\|=\sqrt{1+\bar{a}^{2}}\left\|d-\frac{1}{\sqrt{1+\bar{a}^{2}}}\right\| \geq \sqrt{1+\bar{a}^{2}}\|d-\bar{d}\| .
\end{aligned}
$$


where we applied (50) in the last step. Consequently,

$$
\|d-\bar{d}\| \leq \frac{1}{\sqrt{1+\bar{a}^{2}}}\left\|\bar{a}^{2} d^{2}-c^{2}\right\| \leq \frac{1+\bar{a}}{\sqrt{1+\bar{a}^{2}}}\|\bar{a} d-c\| \leq \sqrt{2}\|\bar{a} d-c\|
$$

and

$$
\|d-\bar{d}\|^{2} \leq 2\|\bar{a} d-c\|^{2} \leq 4\left(\|a d-c\|^{2}+\|a-\bar{a}\|^{2}\right)
$$

using (49).

\section{EEP-inequality and convergence to the equilibrium}

We are now prepared to prove Theorem 1.5.

Proof of Theorem 1.5 Let $\left(n, p, n_{t r}\right) \in L^{1}(\Omega)^{3}$ be non-negative functions satisfying $n_{t r} \leq 1$, the conservation law $\bar{n}-\bar{p}+\varepsilon \overline{n_{t r}}=M$ and the $L^{1}$-bound $\bar{n}, \bar{p} \leq M_{1}$. Keeping in mind that $\nu_{\infty}=\sqrt{n_{*} / n_{0}}$ and $\pi_{\infty}=\sqrt{p_{*} / p_{0}}$ (cf. Notation 5.1), Proposition 4.4 guarantees that there exists a positive constant $C_{1}\left(\gamma, \Gamma, M_{1}\right)>0$ such that

$$
\begin{aligned}
& E\left(n, p, n_{t r}\right)-E\left(n_{\infty}, p_{\infty}, n_{t r, \infty}\right) \leq C_{1}\left(\int_{\Omega}\left(\frac{\left|J_{n}\right|^{2}}{n}+\frac{\left|J_{p}\right|^{2}}{p}\right) d x\right. \\
& \left.+n_{0}\left(\sqrt{\frac{\bar{n}}{n_{0} \mu_{n}}}-v_{\infty}\right)^{2}+p_{0}\left(\sqrt{\overline{\frac{p}{p_{0} \mu_{p}}}}-\pi_{\infty}\right)^{2}+\varepsilon \int_{\Omega}\left(\sqrt{n_{t r}}-\sqrt{n_{t r, \infty}}\right)^{2} d x\right) \text {. }
\end{aligned}
$$

Next, we have to bound the second line of (51) in terms of the entropy production. To this end, we apply Proposition 5.5 with the choices $a:=\sqrt{n /\left(n_{0} \mu_{n}\right)}$, $b:=\sqrt{p /\left(p_{0} \mu_{p}\right)}, c:=\sqrt{n_{t r}}$ and $d:=\sqrt{n_{t r}^{\prime}}$ (as always $n_{t r}^{\prime}=1-n_{t r}$ ). The hypotheses of this proposition are fulfilled as a consequence of the conservation law $\bar{n}-\bar{p}+\varepsilon \overline{n_{t r}}=M$ and the $L^{1}$-bound $\bar{n}, \bar{p} \leq M_{1}$. As a result, we obtain 


$$
\begin{aligned}
& \left(\sqrt{\frac{\bar{n}}{n_{0} \mu_{n}}}-v_{\infty}\right)^{2}+\left(\sqrt{\frac{\bar{p}}{p_{0} \mu_{p}}}-\pi_{\infty}\right)^{2}+\left\|\sqrt{n_{t r}}-\sqrt{n_{t r, \infty}}\right\|^{2} \\
& \leq C_{2}\left(\left\|\sqrt{\frac{n n_{t r}^{\prime}}{n_{0} \mu_{n}}}-\sqrt{n_{t r}}\right\|^{2}+\left\|\sqrt{\frac{p n_{t r}}{p_{0} \mu_{p}}}-\sqrt{n_{t r}^{\prime}}\right\|^{2}\right. \\
& +\left\|\nabla \sqrt{\frac{n}{n_{0} \mu_{n}}}\right\|^{2}+\left\|\nabla \sqrt{\frac{p}{p_{0} \mu_{p}}}\right\|^{2} \\
& +\left\|\sqrt{\frac{n}{n_{0} \mu_{n}}}-\sqrt{\frac{n}{n_{0} \mu_{n}}}\right\|^{2}+\left\|\sqrt{\frac{p}{p_{0} \mu_{p}}}-\sqrt{\frac{p}{p_{0} \mu_{p}}}\right\|^{2} \\
& \left.+\left\|\sqrt{n_{t r}}-\overline{\sqrt{n_{t r}}}\right\|^{2}+\left\|\sqrt{n_{t r}^{\prime}}-\overline{\sqrt{n_{t r}^{\prime}}}\right\|^{2}\right)
\end{aligned}
$$

for all $\varepsilon \in\left(0, \varepsilon_{0}\right]$ with a constant $C_{2}\left(\varepsilon_{0}, n_{0}, p_{0}, M, M_{1}, V\right)>0$. Thanks to Poincaré's inequality, we are able to bound the second and third line on the right hand side from above:

$$
\begin{aligned}
& \left\|\sqrt{\frac{n}{n_{0} \mu_{n}}}-\sqrt{\frac{n}{n_{0} \mu_{n}}}\right\|^{2} \leq C_{P}\left\|\nabla \sqrt{\frac{n}{n_{0} \mu_{n}}}\right\|^{2} \\
& =C_{P} \int_{\Omega}\left|\frac{1}{2} \sqrt{\frac{\mu_{n}}{n_{0} n}} \nabla\left(\frac{n}{\mu_{n}}\right)\right|^{2} d x \leq \frac{C_{P}}{4 n_{0} \inf _{\Omega} \mu_{n}} \int_{\Omega} \frac{\left|J_{n}\right|^{2}}{n} d x
\end{aligned}
$$

and

$$
\left\|\sqrt{\frac{p}{p_{0} \mu_{p}}}-\sqrt{\frac{p}{p_{0} \mu_{p}}}\right\|^{2} \leq C_{P}\left\|\nabla \sqrt{\frac{p}{p_{0} \mu_{p}}}\right\|^{2} \leq \frac{C_{P}}{4 p_{0} \inf _{\Omega} \mu_{p}} \int_{\Omega} \frac{\left|J_{p}\right|^{2}}{p} d x .
$$

Moreover, the elementary inequality $(\sqrt{x}-1)^{2} \leq(x-1) \ln (x)$ for $x>0$ gives rise to

$$
\begin{aligned}
\left\|\sqrt{\frac{n n_{t r}^{\prime}}{n_{0} \mu_{n}}}-\sqrt{n_{t r}}\right\|^{2} & =\int_{\Omega} n_{t r}\left(\sqrt{\frac{n n_{t r}^{\prime}}{n_{0} \mu_{n} n_{t r}}}-1\right)^{2} d x \\
& \leq \int_{\Omega} n_{t r}\left(\frac{n n_{t r}^{\prime}}{n_{0} \mu_{n} n_{t r}}-1\right) \ln \left(\frac{n n_{t r}^{\prime}}{n_{0} \mu_{n} n_{t r}}\right) d x \\
& =\int_{\Omega}\left(\frac{n\left(1-n_{t r}\right)}{n_{0} \mu_{n}}-n_{t r}\right) \ln \left(\frac{n\left(1-n_{t r}\right)}{n_{0} \mu_{n} n_{t r}}\right) d x \\
& =\tau_{n} \int_{\Omega}\left(-R_{n}\right) \ln \left(\frac{n\left(1-n_{t r}\right)}{n_{0} \mu_{n} n_{t r}}\right) d x
\end{aligned}
$$

and similarly 


$$
\left\|\sqrt{\frac{p n_{t r}}{p_{0} \mu_{p}}}-\sqrt{n_{t r}^{\prime}}\right\|^{2} \leq \tau_{p} \int_{\Omega}\left(-R_{p}\right) \ln \left(\frac{p n_{t r}}{p_{0} \mu_{p}\left(1-n_{t r}\right)}\right) d x .
$$

Proposition 5.6 further implies that

$$
\begin{aligned}
& \left\|\sqrt{n_{t r}}-\overline{\sqrt{n_{t r}}}\right\|^{2}+\left\|\sqrt{n_{t r}^{\prime}}-\sqrt{\sqrt{n_{t r}^{\prime}}}\right\|^{2} \\
& \leq 4\left\|\sqrt{\frac{n}{n_{0} \mu_{n}}}-\sqrt{\frac{n}{n_{0} \mu_{n}}}\right\|^{2}+\left\|\sqrt{\frac{p}{p_{0} \mu_{p}}}-\sqrt{\frac{p}{p_{0} \mu_{p}}}\right\|^{2} \\
& \left.\quad+\left\|\sqrt{\frac{n n_{t r}^{\prime}}{n_{0} \mu_{n}}}-\sqrt{n_{t r}}\right\|^{2}+\left\|\sqrt{\frac{p n_{t r}}{p_{0} \mu_{p}}}-\sqrt{n_{t r}^{\prime}}\right\|^{2}\right) .
\end{aligned}
$$

Combining the above estimates, we arrive at

$$
\begin{aligned}
& \left(\sqrt{\frac{\bar{n}}{n_{0} \mu_{n}}}-v_{\infty}\right)^{2}+\left(\sqrt{\frac{p}{p_{0} \mu_{p}}}-\pi_{\infty}\right)^{2}+\left\|\sqrt{n_{t r}}-\sqrt{n_{t r, \infty}}\right\|^{2} \\
& \leq C_{3} \int_{\Omega}\left(\frac{\left|J_{n}\right|^{2}}{n}+\frac{\left|J_{p}\right|^{2}}{p}-R_{n} \ln \left(\frac{n\left(1-n_{t r}\right)}{n_{0} \mu_{n} n_{t r}}\right)-R_{p} \ln \left(\frac{p n_{t r}}{p_{0} \mu_{p}\left(1-n_{t r}\right)}\right)\right)
\end{aligned}
$$

with a constant $C_{3}\left(\varepsilon_{0}, \tau_{n}, \tau_{p}, n_{0}, p_{0}, M, M_{1}, V\right)>0$ uniformly for $\varepsilon \in\left(0, \varepsilon_{0}\right]$. With respect to (51), we now find

$$
\begin{aligned}
& n_{0}\left(\sqrt{\frac{\bar{n}}{n_{0} \mu_{n}}}-v_{\infty}\right)^{2}+p_{0}\left(\sqrt{\frac{\bar{p}}{p_{0} \mu_{p}}}-\pi_{\infty}\right)^{2}+\varepsilon \int_{\Omega}\left(\sqrt{n_{t r}}-\sqrt{n_{t r, \infty}}\right)^{2} d x \\
& \leq \max \left\{n_{0}, p_{0}, \varepsilon_{0}\right\}\left(\sqrt{\frac{n}{n_{0} \mu_{n}}}-v_{\infty}\right)^{2} \\
&\left.+\left(\sqrt{\frac{p}{p_{0} \mu_{p}}}-\pi_{\infty}\right)^{2}+\left\|\sqrt{n_{t r}}-\sqrt{n_{t r, \infty}}\right\|^{2}\right) \\
& \leq C_{3} \max \left\{n_{0}, p_{0}, \varepsilon_{0}\right\} \int_{\Omega}\left(\frac{\left|J_{n}\right|^{2}}{n}+\frac{\left|J_{p}\right|^{2}}{p}-R_{n} \ln \left(\frac{n\left(1-n_{t r}\right)}{n_{0} \mu_{n} n_{t r}}\right)\right. \\
&\left.-R_{p} \ln \left(\frac{p n_{t r}}{p_{0} \mu_{p}\left(1-n_{t r}\right)}\right)\right) .
\end{aligned}
$$

And since the constant $C_{1}$ in (51) only depends on $\varepsilon_{0}, n_{0}, p_{0}, M, M_{1}$ and $V$ (via the constants $\gamma$ and $\Gamma$ ), we have finally proven that 


$$
\begin{aligned}
& E\left(n, p, n_{t r}\right)-E\left(n_{\infty}, p_{\infty}, n_{t r, \infty}\right) \\
& \quad \leq C_{4} \int_{\Omega}\left(\frac{\left|J_{n}\right|^{2}}{n}+\frac{\left|J_{p}\right|^{2}}{p}-R_{n} \ln \left(\frac{n\left(1-n_{t r}\right)}{n_{0} \mu_{n} n_{t r}}\right)-R_{p} \ln \left(\frac{p n_{t r}}{p_{0} \mu_{p}\left(1-n_{t r}\right)}\right)\right) d x
\end{aligned}
$$

for a constant $C_{4}\left(\varepsilon_{0}, \tau_{n}, \tau_{p}, n_{0}, p_{0}, M, M_{1}, V\right)>0$ independent of $\varepsilon \in\left(0, \varepsilon_{0}\right]$.

Theorem 1.5 provides an upper bound for the relative entropy in terms of the entropy production. This already implies exponential convergence of the relative entropy. The subsequent proposition now yields a lower bound for the relative entropy involving the $L^{1}$-distance of the solution to the equilibrium. This will allow us to establish exponential convergence in $L^{1}$.

Proposition 6.1 (Csiszár-Kullback-Pinsker inequality) Let $\varepsilon_{0}, n_{0}, p_{0}, M, M_{1}$ and $V$ be positive constants. Then, there exists an explicit constant $C_{\mathrm{CKP}}>0$ such that for all $\varepsilon \in\left(0, \varepsilon_{0}\right]$, the equilibrium $\left(n_{\infty}, p_{\infty}, n_{t r, \infty}\right) \in X$ from Theorem 2.1 and all nonnegative functions $\left(n, p, n_{t r}\right) \in L^{1}(\Omega)^{3}$ satisfying $n_{t r} \leq 1$, the conservation law

$$
\bar{n}-\bar{p}+\varepsilon \overline{n_{t r}}=M
$$

and the $L^{1}$-bound

$$
\bar{n}, \bar{p} \leq M_{1},
$$

the following Csiszár-Kullback-Pinsker-type inequality holds true:

$$
\begin{aligned}
& E\left(n, p, n_{t r}\right)-E\left(n_{\infty}, p_{\infty}, n_{t r, \infty}\right) \\
& \quad \geq C_{\mathrm{CKP}}\left(\left\|n-n_{\infty}\right\|_{L^{1}(\Omega)}^{2}+\left\|p-p_{\infty}\right\|_{L^{1}(\Omega)}^{2}+\varepsilon\left\|n_{t r}-n_{t r, \infty}\right\|_{L^{2}(\Omega)}^{2}\right) .
\end{aligned}
$$

Proof Due to Lemma 3.2, we know that the relative entropy reads

$$
\begin{aligned}
E\left(n, p, n_{t r}\right)-E\left(n_{\infty}, p_{\infty}, n_{t r, \infty}\right) & \\
= & \int_{\Omega}\left(n \ln \frac{n}{n_{\infty}}-\left(n-n_{\infty}\right)+p \ln \frac{p}{p_{\infty}}-\left(p-p_{\infty}\right)\right. \\
& \left.+\varepsilon \int_{n_{t r, \infty}}^{n_{t r}}\left(\ln \left(\frac{s}{1-s}\right)-\ln \left(\frac{n_{t r, \infty}}{1-n_{t r, \infty}}\right)\right) d s\right) d x .
\end{aligned}
$$

Similar to Proposition 4.4, we employ the mean-value theorem and observe that

$$
\frac{d}{d s} \ln \left(\frac{s}{1-s}\right)=\frac{1}{s(1-s)} \geq 4
$$

for all $s \in(0,1)$. Thus, there exists some $\sigma(s)$ between $n_{t r, \infty}$ and $s$ such that 


$$
\begin{aligned}
& \varepsilon \int_{\Omega} \int_{n_{t r, \infty}}^{n_{t r}}\left(\ln \left(\frac{s}{1-s}\right)-\ln \left(\frac{n_{t r, \infty}}{1-n_{t r, \infty}}\right)\right) d s d x \\
& =\varepsilon \int_{\Omega} \int_{n_{t r, \infty}}^{n_{t r}} \frac{1}{\sigma(s)(1-\sigma(s))}\left(s-n_{t r, \infty}\right) d s d x \\
& \quad \geq 4 \varepsilon \int_{\Omega} \int_{n_{t r, \infty}}^{n_{t r}}\left(s-n_{t r, \infty}\right) d s d x=2 \varepsilon \int_{\Omega}\left(n_{t r}-n_{t r, \infty}\right)^{2} d x
\end{aligned}
$$

Moreover, we utilise the Csiszár-Kullback-Pinsker-inequality from Lemma 3.3 to estimate

$$
\int_{\Omega}\left(n \ln \left(\frac{n}{n_{\infty}}\right)-\left(n-n_{\infty}\right)\right) d x \geq \frac{3}{2 \bar{n}+4 \overline{n_{\infty}}}\left\|n-n_{\infty}\right\|_{L^{1}(\Omega)}^{2} \geq c\left\|n-n_{\infty}\right\|_{L^{1}(\Omega)}^{2}
$$

where $c\left(\varepsilon_{0}, n_{0}, p_{0}, M, M_{1}, V\right)>0$ is a positive constant independent of $\varepsilon \in\left(0, \varepsilon_{0}\right]$. As a corresponding estimate holds true also for $p$, we have verified that

$$
\begin{aligned}
& E\left(n, p, n_{t r}\right)-E\left(n_{\infty}, p_{\infty}, n_{t r, \infty}\right) \\
& \quad \geq C\left(\left\|n-n_{\infty}\right\|_{L^{1}(\Omega)}^{2}+\left\|p-p_{\infty}\right\|_{L^{1}(\Omega)}^{2}+\varepsilon\left\|n_{t r}-n_{t r, \infty}\right\|_{L^{2}(\Omega)}^{2}\right)
\end{aligned}
$$

for some $C\left(\varepsilon_{0}, n_{0}, p_{0}, M, M_{1}, V\right)>0$ uniformly for $\varepsilon \in\left(0, \varepsilon_{0}\right]$.

Now, we are able to prove exponential convergence in relative entropy and $L^{1}$.

Proof of Theorem 1.3 We first prove exponential convergence of the relative entropy

$$
\psi(t):=E\left(n, p, n_{t r}\right)(t)-E\left(n_{\infty}, p_{\infty}, n_{t r, \infty}\right)
$$

using a Gronwall argument as stated in [25]. To this end, we choose $0<t_{0} \leq t_{1} \leq t<T$ and rewrite the entropy production law as

$$
\psi\left(t_{1}\right)-\psi(t)=\int_{t_{1}}^{t} P\left(n, p, n_{t r}\right)(s) d s \geq K \int_{t_{1}}^{t} \psi(s) d s
$$

where we applied Theorem 1.5 with $K:=C_{\mathrm{EEP}}^{-1}$ in the second step. Furthermore, we set

$$
\Psi\left(t_{1}\right):=\int_{t_{1}}^{t} \psi(s) d s=-\int_{t}^{t_{1}} \psi(s) d s
$$

and obtain from (52) the estimate $K \Psi\left(t_{1}\right) \leq \psi\left(t_{1}\right)-\psi(t)$ which yields

$$
\frac{d}{d t_{1}}\left(\Psi\left(t_{1}\right) e^{K t_{1}}\right)=-\psi\left(t_{1}\right) e^{K t_{1}}+K \Psi\left(t_{1}\right) e^{K t_{1}} \leq-\psi(t) e^{K t_{1}}
$$

Integrating this inequality from $t_{1}=t_{0}$ to $t_{1}=t$ and observing that $\Psi(t)=0$ gives rise to 


$$
-\Psi\left(t_{0}\right) e^{K t_{0}} \leq-\frac{\psi(t)}{K}\left(e^{K t}-e^{K t_{0}}\right)
$$

As a consequence of (52) with $t_{1}=t_{0}$, one has $-\Psi\left(t_{0}\right) \geq\left(\psi(t)-\psi\left(t_{0}\right)\right) / K$ and, hence,

$$
-\psi\left(t_{0}\right) e^{K t_{0}} \leq-\psi(t) e^{K t}
$$

But this is equivalent to

$$
E\left(n, p, n_{t r}\right)(t)-E\left(n_{\infty}, p_{\infty}, n_{t r, \infty}\right) \leq\left(E\left(n, p, n_{t r}\right)\left(t_{0}\right)-E_{\infty}\right) e^{-K\left(t-t_{0}\right)},
$$

for all $t \geq t_{0}>0$. In order to conclude that

$$
E\left(n, p, n_{t r}\right)(t)-E\left(n_{\infty}, p_{\infty}, n_{t r, \infty}\right) \leq\left(E_{I}-E_{\infty}\right) e^{-K t},
$$

for all $t \geq 0$, we observe that the rate $K$ is independent of $t_{0}$ and that the entropy $E\left(n, p, n_{t r}\right)\left(t_{0}\right)$ extends in (53) continuously to $t_{0} \rightarrow 0$ since $n, p \in C\left([0, T) ; L^{2}(\Omega)\right)$ for all $T>0$ by Theorem 1.1. This results in the announced exponential decay of the relative entropy, while the exponential convergence in $L^{1}$ follows from Proposition 6.1 .

Proof of Corollary 1.8 We first prove that the linearly growing $L^{\infty}$-bounds together with parabolic regularity for system (1) and assumption (4) entail polynomially growing $W^{1, q}$-bounds, $q \in(1, \infty)$, for $n$ and $p$. To this end, we consider

$$
\partial_{t} n=\nabla \cdot J_{n}+\frac{1}{\tau_{n}}\left(n_{t r}-\frac{n}{n_{0} e^{-V_{n}}}\left(1-n_{t r}\right)\right), \quad J_{n}=e^{-V_{n}} \nabla\left(n e^{V_{n}}\right),
$$

and introduce the variable $w=n e^{V_{n}}$. We observe that $\nabla \cdot J_{n}=\nabla \cdot\left(e^{-V_{n}} \nabla w\right)=e^{-V_{n}}\left(\Delta w-\nabla V_{n} \cdot \nabla w\right)$ and thus,

$$
\partial_{t} w=\Delta w-\nabla V_{n} \cdot \nabla w+\frac{e^{V_{n}}}{\tau_{n}}\left(n_{t r}-\frac{1-n_{t r}}{n_{0}} w\right) .
$$

Under the assumptions of Corollary 1.8, this equation is of the form

$$
\partial_{t} w-\Delta w=f_{1}+f_{2} w+f_{3} \cdot \nabla w
$$

where $f_{i} \in C\left([0, \infty), L^{\infty}(\Omega)\right)$ for $i \in\{1,2\}, f_{3} \in C\left([0, \infty), L^{\infty}(\Omega)^{m}\right)$ and $\hat{n} \cdot \nabla w=0$ on $\partial \Omega$. Testing this equation with $-(q-1)|\nabla w|^{q-2} \Delta w$ yields 


$$
\begin{aligned}
\frac{1}{q} \frac{d}{d t} & \int_{\Omega}|\nabla w|^{q} d x=\int_{\Omega}|\nabla w|^{q-2} \nabla w \cdot \nabla \partial_{t} w d x \\
= & -\int_{\Omega}\left((q-2)|\nabla w|^{q-4} \nabla w \Delta w \cdot \nabla w+|\nabla w|^{q-2} \Delta w\right) \partial_{t} w d x \\
= & -\int_{\Omega}(q-1)|\nabla w|^{q-2} \Delta w \partial_{t} w d x \\
= & -\int_{\Omega}(q-1)|\nabla w|^{q-2}|\Delta w|^{2} d x \\
& -\int_{\Omega}(q-1)|\nabla w|^{q-2} \Delta w\left(f_{1}+f_{2} w+f_{3} \cdot \nabla w\right) d x
\end{aligned}
$$

Using the inequalities $|a b| \leq\left(a^{2}+b^{2}\right) / 2$ and $(a+b+c)^{2} \leq 3\left(a^{2}+b^{2}+c^{2}\right)$ for $a, b, c \in \mathbb{R}$, we find

$$
\begin{aligned}
& \frac{1}{q} \frac{d}{d t} \int_{\Omega}|\nabla w|^{q} d x \leq-\frac{1}{2} \int_{\Omega}(q-1)|\nabla w|^{q-2}|\Delta w|^{2} d x \\
& \quad+\frac{3}{2} \int_{\Omega}(q-1)|\nabla w|^{q-2}\left(f_{1}^{2}+f_{2}^{2} w^{2}+f_{3}^{2}|\nabla w|^{2}\right) d x .
\end{aligned}
$$

Together with $C>0$ satisfying $\left|f_{i}(t, x)^{2}\right| \leq C$ for all $i \in\{1,2,3\}, t \geq 0$ and a.e. $x \in \Omega$, we derive

$$
\begin{aligned}
& \frac{1}{q} \frac{d}{d t} \int_{\Omega}|\nabla w|^{q} d x \leq-\frac{1}{2} \int_{\Omega}(q-1)|\nabla w|^{q-2}|\Delta w|^{2} d x \\
& \quad+\frac{3 C}{2} \int_{\Omega}(q-1)|\nabla w|^{q-2}\left(1+w^{2}+|\nabla w|^{2}\right) d x .
\end{aligned}
$$

An integration by parts and Young's inequality with $C_{1}(C, q)>0$ give rise to

$$
\begin{aligned}
\int_{\Omega} & (q-1)|\nabla w|^{q-2} \nabla w \cdot \nabla w d x \\
\quad & -\int_{\Omega}\left((q-1)(q-2)|\nabla w|^{q-4} \nabla w \Delta w \cdot \nabla w+(q-1)|\nabla w|^{q-2} \Delta w\right) w d x \\
& =-\int_{\Omega}(q-1)^{2}|\nabla w|^{q-2} \Delta w w d x \\
& \leq \frac{1}{3 C} \int_{\Omega}(q-1)^{1}|\nabla w|^{q-2}|\Delta w|^{2} d x+C_{1} \int_{\Omega}|\nabla w|^{q-2} w^{2} d x .
\end{aligned}
$$

Hence, there exists a constant $C_{2}(C, q)>0$ such that

$$
\frac{d}{d t} \int_{\Omega}|\nabla w|^{q} d x \leq C_{2} \int_{\Omega}|\nabla w|^{q-2}\left(1+w^{2}\right) d x \leq\left(A+B t^{2}\right) \int_{\Omega}|\nabla w|^{q-2} d x,
$$

where $A, B>0$ result from the linearly growing $L^{\infty}$-bounds from (11). For any fixed $t_{0}>0$ and all $t \geq t_{0}$, we now have 


$$
\|\nabla w(t)\|_{L^{q}(\Omega)}^{q} \leq\left\|\nabla w\left(t_{0}\right)\right\|_{L^{q}(\Omega)}^{q}+\int_{t_{0}}^{t}\left(A+B s^{2}\right)\|\nabla w(s)\|_{L^{q}(\Omega)}^{q-2} d s .
$$

A Gronwall lemma (see e.g. [2]) now proves the desired polynomial growth of $\|\nabla w\|_{L^{q}(\Omega)}$ and $\|\nabla n\|_{L^{q}(\Omega)}$ :

$$
\|\nabla w(t)\|_{L^{q}(\Omega)} \leq\left(\left\|\nabla w\left(t_{0}\right)\right\|_{L^{q}(\Omega)}^{2}+A\left(t-t_{0}\right)+\frac{B}{3}\left(t^{3}-t_{0}^{3}\right)\right)^{\frac{1}{2}} .
$$

Next, we use the Gagliardo-Nirenberg-Moser interpolation inequality in $\mathbb{R}^{m}, m \geq 1$ (see e.g. [23]):

$$
\left\|n-n_{\infty}\right\|_{L^{\infty}(\Omega)} \leq G(\Omega)\left\|n-n_{\infty}\right\|_{W^{1,2 m}(\Omega)}^{\frac{1}{2}}\left\|n-n_{\infty}\right\|_{L^{2 m}(\Omega)}^{\frac{1}{2}} .
$$

Then, interpolating with the exponentially decaying $L^{1}$-norm of $n-n_{\infty}$, we obtain

$$
\begin{aligned}
& \|n(t, \cdot)\|_{L^{\infty}(\Omega)} \leq\left\|n_{\infty}\right\|_{L^{\infty}(\Omega)}+\left\|n-n_{\infty}\right\|_{L^{\infty}(\Omega)} \\
& \quad \leq\left\|n_{\infty}\right\|_{L^{\infty}(\Omega)}+G\left\|n-n_{\infty}\right\|_{W^{1,2 m}(\Omega)}^{\frac{1}{2}}\left\|n-n_{\infty}\right\|_{L^{\infty}(\Omega)}^{\frac{1}{2}-\frac{1}{4 m}}\left\|n-n_{\infty}\right\|_{L^{1}(\Omega)}^{\frac{1}{4 m}} \leq Z
\end{aligned}
$$

due to the exponential convergence to equilibrium (15). The estimate for $p$ follows in the same way.

Proof of Corollary 1.9 We first notice that exponential convergence of $n$ and $p$ in $L^{q}(\Omega), 1<q<\infty$, immediately follows from Theorem 1.3 and Corollary 1.8 via

$$
\left\|n-n_{\infty}\right\|_{L^{q}(\Omega)}^{q} \leq\left\|n-n_{\infty}\right\|_{L^{\infty}(\Omega)}^{q-1}\left\|n-n_{\infty}\right\|_{L^{1}(\Omega)} \leq C_{q} e^{-K_{q} t}
$$

and an analogous estimate for $p$ where $0<C_{q}, K_{q}<\infty$ are constants independent of $\varepsilon \in\left(0, \varepsilon_{0}\right]$. Reusing the Gagliardo-Nirenberg-Moser interpolation inequality in $\mathbb{R}^{m}, m \geq 1$, from (56) and the polynomial bound on the growth of $\|\nabla n(t)\|_{L^{2 m}(\Omega)}$ from (55), we derive

$$
\left\|n-n_{\infty}\right\|_{L^{\infty}(\Omega)} \leq G(\Omega)\left\|n-n_{\infty}\right\|_{W^{1,2 m}(\Omega)}^{\frac{1}{2}}\left\|n-n_{\infty}\right\|_{L^{2 m}(\Omega)}^{\frac{1}{2}} \leq C_{m} e^{-K_{m} t},
$$

thus, establishing exponential convergence of $n$ and $p$ in $L^{\infty}(\Omega)$. Concerning the convergence of $n_{t r}$, we recall the following identities from (25) and (22):

$$
1-n_{t r, \infty}=\frac{p_{\infty}}{p_{0} \mu_{p}} n_{t r, \infty}, \quad n_{t r, \infty}=\frac{n_{\infty}}{n_{0} \mu_{n}}\left(1-n_{t r, \infty}\right), \quad \frac{p_{\infty}}{\mu_{p}}=p_{*}, \quad \frac{n_{\infty}}{\mu_{n}}=n_{*} .
$$

We abbreviate $u:=n_{t r}-n_{t r, \infty}$ and calculate (pointwise in $x$ ) by adding and subtracting $n_{t r, \infty}$ and $n_{t r}$ multiple times and by using the relations (58): 


$$
\begin{aligned}
\varepsilon \partial_{t} u= & R_{p}-R_{n}=\frac{1}{\tau_{p}}\left(1-n_{t r}-\frac{p}{p_{0} \mu_{p}} n_{t r}\right)-\frac{1}{\tau_{n}}\left(n_{t r}-\frac{n}{n_{0} \mu_{n}}\left(1-n_{t r}\right)\right) \\
= & \frac{1}{\tau_{p}}\left(-u+\frac{p_{\infty}}{p_{0} \mu_{p}} n_{t r, \infty}-\frac{p}{p_{0} \mu_{p}} n_{t r}\right) \\
& -\frac{1}{\tau_{n}}\left(u+\frac{n_{\infty}}{n_{0} \mu_{n}}\left(1-n_{t r, \infty}\right)-\frac{n}{n_{0} \mu_{n}}\left(1-n_{t r}\right)\right) \\
= & -u \underbrace{\left(\frac{1}{\tau_{p}}+\frac{p_{*}}{\tau_{p} p_{0}}+\frac{1}{\tau_{n}}+\frac{n_{*}}{\tau_{n} n_{0}}\right)}_{=: K} \\
& -\frac{n_{t r}}{\tau_{p} p_{0} \mu_{p}}\left(p-p_{\infty}\right)+\frac{\left(1-n_{t r}\right)}{\tau_{n} n_{0} \mu_{n}}\left(n-n_{\infty}\right) .
\end{aligned}
$$

Hence, due to $0 \leq n_{t r}(t, x) \leq 1$ for all $t \geq 0$ and a.e. $x \in \Omega, \mu_{n}=e^{-V_{n}}, \mu_{p}=e^{-V_{p}}$, and $V=\max \left(\left\|V_{n}\right\|_{L^{\infty}(\Omega)},\left\|V_{p}\right\|_{L^{\infty}(\Omega)}\right)$, we estimate with (57)

$$
\begin{aligned}
\frac{d}{d t}\|u(t, \cdot)\|_{L^{\infty}(\Omega)} \leq- & \frac{K}{\varepsilon}\|u(t, \cdot)\|_{L^{\infty}(\Omega)}+\frac{e^{V}}{\varepsilon \tau_{p} p_{0}}\left\|p(t, \cdot)-p_{\infty}\right\|_{L^{\infty}(\Omega)} \\
& \quad+\frac{e^{V}}{\varepsilon \tau_{n} n_{0}}\left\|n(t, \cdot)-n_{\infty}\right\|_{L^{\infty}(\Omega)} \\
\leq- & \frac{K}{\varepsilon}\|u(t, \cdot)\|_{L^{\infty}(\Omega)}+\frac{C}{\varepsilon} e^{-K_{m} t}
\end{aligned}
$$

where $C:=C_{m} e^{V}\left(\left(\tau_{p} p_{0}\right)^{-1}+\left(\tau_{n} n_{0}\right)^{-1}\right)$. For the following calculation, we assume w.l.o.g. $2 \varepsilon_{0} K_{m} \leq K$. Hence, using $\|u(0, \cdot)\|_{L^{\infty}(\Omega)} \leq 1$, we arrive at

$$
\begin{aligned}
& \left\|n_{t r}(t, \cdot)-n_{t r, \infty}\right\|_{L^{\infty}(\Omega)} \leq e^{-K t / \varepsilon}+\frac{C}{\varepsilon} \int_{0}^{t} e^{-K(t-s) / \varepsilon-K_{m} s} d s \\
& \quad \leq e^{-K t / \varepsilon}+e^{-K t / \varepsilon} \frac{C}{K-\varepsilon K_{m}}\left(e^{\left(K / \varepsilon-K_{m}\right) t}-1\right) \leq e^{-K t / \varepsilon_{0}}+\frac{C}{K-\varepsilon_{0} K_{m}} e^{-K_{m} t},
\end{aligned}
$$

where the last bound is independent of $\varepsilon$.

\section{A limiting entropy method for system (20)}

The existence theory of the Shockley-Read-Hall model applies classical methods (see e.g. [18]) and can be carried out analogously to Theorem 1.1. Therefore, we state here the corresponding results without proof.

Theorem 7.1 (Shockley-Read-Hall for $\varepsilon=0$ ) Under the assumptions of Theorem 1.1, there exists a unique non-negative global weak solution $(n, p) \in\left(C\left([0, T], L^{2}(\Omega)\right) \cap W_{2}(0, T) \cap L^{\infty}\left((0, T), L^{\infty}(\Omega)\right)\right)^{2}$, of system (20) for all $T \in(0, \infty)$ satisfying the boundary conditions $(2)$. 
Moreover, there exist positive constants $C_{n}\left(\left\|n_{I}\right\|_{L^{\infty}(\Omega)}, V_{n}\right), C_{p}\left(\left\|p_{I}\right\|_{L^{\infty}(\Omega)}, V_{p}\right)$ and $K_{n}\left(V_{n}\right), K_{p}\left(V_{p}\right)$ such that

$$
\|n(t, \cdot)\|_{L^{\infty}(\Omega)} \leq C_{n}+K_{n} t, \quad\|p(t, \cdot)\|_{L^{\infty}(\Omega)} \leq C_{p}+K_{p} t, \quad \text { for all } t \geq 0 .
$$

Finally, there exist positive constants $\mu, \Gamma, \theta>0$ (depending on $\tau, C_{n}, C_{p}, K_{n}, K_{p}, V_{n}$, $\left.V_{p}\right)$ such that

$$
n(t, x), p(t, x) \geq \min \left\{\mu t, \frac{\Gamma}{1+\theta t}\right\} \quad \text { for all } t \geq 0 \text { and a.e. } x \in \Omega
$$

where $\mu \tau=\frac{\Gamma}{1+\theta \tau}$ such that the bounds $\mu$ t and $\Gamma /(1+\theta t)$ intersect at time $\tau$.

The entropy functional (5) extends continuously to the limit $\varepsilon=0$ :

$$
E_{0}(n, p):=\int_{\Omega}\left(n \ln \frac{n}{n_{0} \mu_{n}}-\left(n-n_{0} \mu_{n}\right)+p \ln \frac{p}{p_{0} \mu_{p}}-\left(p-p_{0} \mu_{p}\right)\right) d x,
$$

which is again an entropy (the free energy) functional of the Shockley-Read-Hall model (20). The corresponding entropy production (free energy dissipation) functional reads as

$$
P_{0}(n, p):=-\frac{d}{d t} E_{0}(n, p)=\int_{\Omega}\left(\frac{\left|J_{n}\right|^{2}}{n}+\frac{\left|J_{p}\right|^{2}}{p}-R \ln \left(\frac{n p}{n_{0} \mu_{n} p_{0} \mu_{p}}\right)\right) d x \geq 0 .
$$

Next, we recall from the introduction $n_{t r}^{q s s a}=n_{t r}^{q s s a}(n, p)$ such that $R_{n}\left(n, n_{t r}^{q s s a}\right)=R_{p}\left(p, n_{t r}^{q s s a}\right)$, i.e.

$$
n_{t r}^{q s s a}:=\frac{\tau_{n}+\tau_{p} \frac{n}{n_{0} \mu_{n}}}{\tau_{n}+\tau_{p}+\tau_{n} \frac{p}{p_{0} \mu_{p}}+\tau_{p} \frac{n}{n_{0} \mu_{n}}} .
$$

$n_{t r}^{q s s a}(n, p)$ denotes the pointwise equilibrium value of the trapped states in (1) for fixed $n$ and $p$, which corresponds to the quasi-steady-state approximation $\varepsilon=0$.

Moreover, we observe that the Shockley-Read-Hall entropy production functional (61) can be identified as the entropy production functional $P\left(n, p, n_{t r}^{q s s a}\right)$ as given in (7) along trajectories of (1) with $\varepsilon=0$ when $n_{t r} \equiv n_{t r}^{q s s a}(n, p)$ :

$$
\begin{aligned}
P\left(n, p, n_{t r}^{q s s a}\right)= & \int_{\Omega}\left(\frac{\left|J_{n}\right|^{2}}{n}+\frac{\left|J_{p}\right|^{2}}{p}-R_{n} \ln \left(\frac{n\left(1-n_{t r}^{q s s a}\right)}{n_{0} \mu_{n} n_{t r}^{q s s a}}\right)\right. \\
& \left.-R_{p} \ln \left(\frac{p n_{t r}^{q s s a}}{p_{0} \mu_{p}\left(1-n_{t r}^{q s s a}\right)}\right)\right) d x \\
= & \int_{\Omega}\left(\frac{\left|J_{n}\right|^{2}}{n}+\frac{\left|J_{p}\right|^{2}}{p}-R \ln \left(\frac{n p}{n_{0} \mu_{n} p_{0} \mu_{p}}\right)\right) d x=P_{0}(n, p)
\end{aligned}
$$


where one uses $R=R_{n}=R_{p}$ at $n_{t r}=n_{t r}^{q s s a}$ and that the involved integrals are finite.

Analogously to Theorem 2.1, there exists a unique equilibrium $\left(n_{\infty, 0}, p_{\infty, 0}\right) \in X_{0}$ in the case $\varepsilon=0$, where

$$
\begin{aligned}
X_{0}:=\left\{(n, p) \in H^{1}(\Omega)^{2} \mid \bar{n}-\bar{p}=M\right. \\
\left.\quad \wedge(\exists \gamma>0) n, p \geq \gamma \text { a.e. } \wedge n_{t r}^{q s s a} \in[\gamma, 1-\gamma] \text { a.e. }\right\} .
\end{aligned}
$$

This equilibrium reads

$$
n_{\infty, 0}=n_{*, 0} e^{-V_{n}}, \quad p_{\infty, 0}=p_{*, 0} e^{-V_{p}},
$$

where $n_{*, 0}, p_{*, 0}>0$ are uniquely determined by $n_{*, 0} p_{*, 0}=n_{0} p_{0}$ and $n_{*, 0} \overline{\mu_{n}}-p_{*, 0} \overline{\mu_{p}}=M$.

We are now in the position to formulate the EEP-inequality.

Theorem 7.2 (Entropy-Entropy Production Inequality for $\varepsilon=0$ ) Let $\tau_{n}, \tau_{p}, n_{0}, p_{0}$, $M_{1}$ and $V$ be positive constants. Consider $M \in \mathbb{R}$ and the correspondingly unique equilibrium $\left(n_{\infty, 0}, p_{\infty, 0}\right) \in X_{0}$.

Then, the following EEP-inequality holds true for all non-negative functions $(n, p) \in L^{1}(\Omega)^{2}$ satisfying the conservation law $\bar{n}-\bar{p}=M$, the $L^{1}$-bound $\bar{n}, \bar{p}<M_{1}$ as well as the conditions $E_{0}(n, p)<\infty, P_{0}(n, p)<\infty, P\left(n, p, n_{t r}^{q s s a}\right)<\infty$ for some $\varepsilon_{0}>0$ :

$$
E_{0}(n, p)-E_{0}\left(n_{\infty, 0}, p_{\infty, 0}\right) \leq C_{\mathrm{EEP}} P_{0}(n, p),
$$

where $C_{\mathrm{EEP}}>0$ is the same constant as in Theorem 1.5 .

Theorem 7.3 (Exponential convergence for $\varepsilon=0)$ Let $(n, p)$ be a global weak solution of system (20) as given in Theorem 7.1 corresponding to the non-negative initial data $\left(n_{I}, p_{I}\right) \in L^{\infty}(\Omega)^{2}$. Then, this solution satisfies the entropy production law

$$
E_{0}(n, p)\left(t_{1}\right)+\int_{t_{0}}^{t_{1}} P_{0}(n, p)(s) d s=E_{0}(n, p)\left(t_{0}\right)
$$

for all $0<t_{0} \leq t_{1}<\infty$.

Moreover, the following versions of the exponential decay towards the equilibrium $\left(n_{\infty, 0}, p_{\infty, 0}\right) \in X_{0}$ hold true:

$$
E_{0}(n, p)(t)-E_{\infty} \leq\left(E_{I}-E_{\infty}\right) e^{-K t}
$$

and

$$
\left\|n-n_{\infty, 0}\right\|_{L^{1}(\Omega)}^{2}+\left\|p-p_{\infty, 0}\right\|_{L^{1}(\Omega)}^{2} \leq C\left(E_{I}-E_{\infty}\right) e^{-K t}
$$


where $C:=C_{\mathrm{CKP}}^{-1}$ and $K:=C_{\mathrm{EEP}}^{-1}$ are the same constants as in Theorem 1.3. Moreover, $E_{I}$ and $E_{\infty}$ denote the initial entropy of the system and the entropy in the equilibrium, respectively.

Remark 7.4 We believe that the entropy-entropy production inequality (64) can also be proven by combining estimates of Sect. 5 with previous works on the entropy method for detailed balanced reaction-diffusion models, see e.g. [5, 7, 10, 19]. We emphasise, however, that our goal with Theorem 7.2 is to be able to derive an entropy-entropy production inequality via the fast-reaction parameter $\varepsilon \rightarrow 0$.

Finally, in the same way as for strictly positive $\varepsilon>0$, we can derive uniformin-time $L^{\infty}$-bounds for $n$ and $p$ also in the case $\varepsilon=0$. As before, these bounds further improve the lower bounds on $n$ and $p$.

Corollary 7.5 There exists a constant $Z>0$ such that

$$
\|n(t, \cdot)\|_{L^{\infty}(\Omega)},\|p(t, \cdot)\|_{L^{\infty}(\Omega)} \leq Z \text { for all } t \geq 0 .
$$

And for all $\tau>0$ there exist sufficiently small constants $\mu, \Gamma>0$ such that

$$
n(t, x), p(t, x) \geq \min \{\mu t, \Gamma\}
$$

for all $t \geq 0$ and a.e. $x \in \Omega$, where $\mu \tau=\Gamma$ such that the bounds $\mu t$ and $\Gamma$ intersect at time $\tau>0$.

Corollary 7.6 Under the hypotheses of Theorem 7.3, there exist constants $0<C, K<\infty$ such that

$$
\left\|n-n_{\infty}\right\|_{L^{\infty}(\Omega)}+\left\|p-p_{\infty}\right\|_{L^{\infty}(\Omega)} \leq C e^{-K t}
$$

holds true for all $t \geq 0$.

Proof of Theorem 1.5 Our goal is to derive an estimate of the form

$$
E_{0}(n, p)-E_{0}\left(n_{\infty, 0}, p_{\infty, 0}\right) \leq C_{E E P} P_{0}(n, p)
$$

by applying the EEP-inequality from Theorem 1.5 directly to the functions $n, p$ and $n_{t r}^{q s s a}$. However, since we assume that $n$ and $p$ satisfy

$$
\bar{n}-\bar{p}=M,
$$

the triple $\left(n, p, n_{t r}^{q s s}\right)$ does not satisfy the conservation law with right hand side $M$ but

$$
\bar{n}-\bar{p}+\varepsilon \overline{n_{t r}^{q s s a}}=M+\varepsilon \overline{n_{t r}^{q s s a}} .
$$

In order to resolve this issue, we shall apply the EEP-inequality from Theorem 1.5 to a suitably defined sequence of functions $\left(n_{\varepsilon}, p_{\varepsilon}, n_{t r, \varepsilon}\right) \in L^{1}(\Omega)^{3}$ which fulfil $\left\|n_{t r, \varepsilon}\right\|_{L^{\infty}(\Omega)} \leq 1$, the $L^{1}$-bound $\overline{n_{\varepsilon}}, \overline{p_{\varepsilon}} \leq M_{1}$ and the conservation law 


$$
\overline{n_{\varepsilon}}-\overline{p_{\varepsilon}}+\varepsilon \overline{n_{t r, \varepsilon}}=M
$$

A convenient choice is $n_{\varepsilon}:=n, \quad p_{\varepsilon}:=p+\varepsilon \overline{n_{t r}^{q s a}}$ and $n_{t r, \varepsilon}:=n_{t r}^{q s s a}$, where $n_{t r}^{q s s a}=n_{t r}^{q s s a}(n, p)$ as defined in (62). For this choice, we derive the stated EEP-estimate for the case $\varepsilon=0$ via the following steps, which are proven below:

$$
\begin{gathered}
E_{0}(n, p)-E_{0}\left(n_{\infty, 0}, p_{\infty, 0}\right)=\lim _{\varepsilon \rightarrow 0}\left(E\left(n_{\varepsilon}, p_{\varepsilon}, n_{t r, \varepsilon}\right)-E\left(n_{\infty}, p_{\infty}, n_{t r, \infty}\right)\right) \\
\leq \lim _{\varepsilon \rightarrow 0}\left(C_{E E P} P\left(n_{\varepsilon}, p_{\varepsilon}, n_{t r, \varepsilon}\right)\right) \\
=C_{E E P} P\left(n, p, n_{t r}^{q s s a}\right)=C_{E E P} P_{0}(n, p)
\end{gathered}
$$

We recall that $n$ and $p$ are assumed to satisfy $E_{0}(n, p)<\infty$ and $P_{0}(n, p), P\left(n, p, n_{t r}^{q s s a}\right)<\infty$, which implies that $P_{0}(n, p)=P\left(n, p, n_{t r}^{q s s a}\right)$ as discussed in the introduction.

Step 1. Proof of (69): We first show, that with $\left(n_{\varepsilon}, p_{\varepsilon}, n_{t r, \varepsilon}\right)=\left(n, p_{\varepsilon}, n_{t r}^{q s s a}\right)$

$$
E_{0}(n, p)=\lim _{\varepsilon \rightarrow 0} E\left(n_{\varepsilon}, p_{\varepsilon}, n_{t r, \varepsilon}\right) .
$$

Recalling that

$$
\begin{aligned}
E\left(n, p_{\varepsilon}, n_{t r}^{q s s}\right)= & \int_{\Omega}\left(n \ln \frac{n}{n_{0} \mu_{n}}-\left(n-n_{0} \mu_{n}\right)+p_{\varepsilon} \ln \frac{p_{\varepsilon}}{p_{0} \mu_{p}}\right. \\
& \left.-\left(p_{\varepsilon}-p_{0} \mu_{p}\right)+\varepsilon \int_{1 / 2}^{n_{t r}^{q s a}} \ln \left(\frac{s}{1-s}\right) d s\right) d x
\end{aligned}
$$

we first notice that $p_{\varepsilon}=p+\varepsilon \overline{n_{t r}^{q s s a}} \rightarrow p$ monotonically decreasing for $\varepsilon \rightarrow 0$ for all $x \in \Omega$. Thus, by using $\overline{n_{t r}^{q s s a}} \leq 1$ and the elementary estimate $p_{\varepsilon} \ln p_{\varepsilon} \leq 2 p(\ln p+\ln 2)$ for $p \geq \max \left\{\varepsilon_{0}, 1\right\}$, the Lebesgue dominated convergence theorem, the $L^{1}$-bounds $\bar{n}, \overline{n_{\varepsilon}}, \bar{p}, \overline{p_{\varepsilon}} \leq M_{1}$ and $E_{0}(n, p)<\infty$ imply the convergence of the $p_{\varepsilon}$-integral in (72). The convergence of the third integral follows directly from

$$
\left|\varepsilon \int_{1 / 2}^{n_{t r}^{q s s a}(x)} \ln \frac{s}{1-s} d s\right| \leq \varepsilon \int_{1 / 2}^{1} \ln \frac{s}{1-s} d s \stackrel{\varepsilon \rightarrow 0}{\longrightarrow} 0
$$

Using analogue arguments, the convergence

$$
E_{0}\left(n_{\infty, 0}, p_{\infty, 0}\right)=\lim _{\varepsilon \rightarrow 0} E\left(n_{\infty}, p_{\infty}, n_{t r, \infty}\right)
$$

follows from observing the monotone convergence $n_{*} \rightarrow n_{*, 0}$ and $p_{*} \rightarrow p_{*, 0}$ for $\varepsilon \rightarrow 0$ due to (27) in the proof of Theorem 2.1 , which directly implies the monotone convergence $n_{\infty} \rightarrow n_{\infty, 0}$ and $p_{\infty} \rightarrow p_{\infty, 0}$ for all $x \in \Omega$, where $\left(n_{\infty}, p_{\infty}, n_{t r, \infty}\right)$ and $\left(n_{\infty, 0}, p_{\infty, 0}\right)$ are defined in (22) and (63), respectively. 
Step 2. Proof of (70): We observe that the functions $\left(n_{\varepsilon}, p_{\varepsilon}, n_{t r, \varepsilon}\right)=\left(n, p+\varepsilon \overline{n_{t r}^{q s s a}}, n_{t r}^{q s s a}\right) \in L^{1}(\Omega)^{3}$ satisfy $\left\|n_{t r, \varepsilon}\right\|_{L^{\infty}(\Omega)} \leq 1$, the conservation law

$$
\bar{n}_{\varepsilon}-\overline{p_{\varepsilon}}+\varepsilon \overline{n_{t r, \varepsilon}}=\bar{n}-\bar{p}=M
$$

as well as the $L^{1}$-bounds $\bar{n}_{\varepsilon} \leq M_{1}$ and $\overline{p_{\varepsilon}} \leq \bar{p}+\varepsilon^{\prime}$ where $\varepsilon \in\left(0, \varepsilon^{\prime}\right] \subset\left(0, \varepsilon_{0}\right]$. Because of $\bar{p}<M_{1}$, we have $\overline{p_{\varepsilon}} \leq M_{1}$ for $\varepsilon^{\prime}>0$ sufficiently small. As a consequence,

$$
E\left(n_{\varepsilon}, p_{\varepsilon}, n_{t r, \varepsilon}\right)-E\left(n_{\infty}, p_{\infty}, n_{t r, \infty}\right) \leq C_{E E P} P\left(n_{\varepsilon}, p_{\varepsilon}, n_{t r, \varepsilon}\right)
$$

where $C_{E E P}>0$ is the same constant as in Theorem 1.5.

Step 3. Proof of (71): As the constant $C_{E E P}>0$ is independent of $\varepsilon \in\left(0, \varepsilon_{0}\right]$, it suffices to show that

$$
\lim _{\varepsilon \rightarrow 0} P\left(n_{\varepsilon}, p_{\varepsilon}, n_{t r, \varepsilon}\right)=P\left(n, p, n_{t r}^{q s s a}\right) .
$$

To this end, we consider the representation

$$
\begin{aligned}
P\left(n_{\varepsilon}, p_{\varepsilon}, n_{t r, \varepsilon}\right)= & \int_{\Omega}\left(\frac{\left|J_{n}\right|^{2}}{n}+\frac{|\nabla p|^{2}}{p_{\varepsilon}}+2 \nabla p \cdot \nabla V_{p}+p_{\varepsilon}\left|\nabla V_{p}\right|^{2}\right. \\
& -R_{n} \ln \left(\frac{n\left(1-n_{t r}^{q s s a}\right)}{n_{0} \mu_{n} n_{t r}^{q s a}}\right)+\frac{1}{\tau_{p}}\left(\frac{p_{\varepsilon}}{p_{0} \mu_{p}} n_{t r}^{q s s a}-\left(1-n_{t r}^{q s s a}\right)\right) \\
& \left.\times\left(\ln \frac{p_{\varepsilon} n_{t r}^{q s s a}}{p_{0} \mu_{p}}-\ln \left(1-n_{t r}^{q s s a}\right)\right)\right) d x,
\end{aligned}
$$

where we have already taken into account that $n_{\varepsilon}=n, \nabla p_{\varepsilon}=\nabla p$ and $n_{t r, \varepsilon}=n_{t r}^{q s s a}$ for all $\varepsilon>0$.

We note first that the convergence of the second, third and forth integral follows from the pointwise convergence of $p_{\varepsilon}$ for all $x \in \Omega$ and from the Lebesgue dominated convergence theorem by estimating

$$
\begin{gathered}
0 \leq \frac{|\nabla p|^{2}}{p_{\varepsilon}}+2 \nabla p \cdot \nabla V_{p}+p_{\varepsilon}\left|\nabla V_{p}\right|^{2} \leq \frac{|\nabla p|^{2}}{p}+2 \nabla p \cdot \nabla V_{p}+p\left|\nabla V_{p}\right|^{2} \\
+\left(p_{\varepsilon}-p\right)\left|\nabla V_{p}\right|^{2} \leq \frac{\left|J_{p}\right|^{2}}{p}+\varepsilon_{0} \overline{n_{t r}^{q s s a}}\left|\nabla V_{p}\right|^{2},
\end{gathered}
$$

where the function on the right hand side is integrable due to the finiteness of $P\left(n, p, n_{t r}^{q s s a}\right)$.

Secondly, the product

$$
\begin{aligned}
& \left(\frac{p_{\varepsilon}}{p_{0} \mu_{p}} n_{t r}^{q s s a}-\left(1-n_{t r}^{q s s a}\right)\right)\left(\ln \frac{p_{\varepsilon} n_{t r}^{q s s a}}{p_{0} \mu_{p}}-\ln \left(1-n_{t r}^{q s s a}\right)\right) \\
& \longrightarrow\left(\frac{p}{p_{0} \mu_{p}} n_{t r}^{q s s a}-\left(1-n_{t r}^{q s s a}\right)\right)\left(\ln \frac{p n_{t r}^{q s s a}}{p_{0} \mu_{p}}-\ln \left(1-n_{t r}^{q s s a}\right)\right)
\end{aligned}
$$


converges pointwise for all $x \in \Omega$ as $\varepsilon \rightarrow 0$. In order to conclude the convergence of the corresponding integral via the Lebesgue dominated convergence theorem, we use similar to Step 1 the elementary inequality $p_{\varepsilon} \ln p_{\varepsilon} \leq 2 p(\ln p+\ln 2)$ for $p \geq \max \left\{\varepsilon_{0}, 1\right\}$ and the finiteness of $P\left(n, p, n_{t r}^{q s s a}\right)$. This yields

$$
\begin{aligned}
& \lim _{\varepsilon \rightarrow 0} \int_{\Omega} \frac{1}{\tau_{p}}\left(\frac{p_{\varepsilon}}{p_{0} \mu_{p}} n_{t r}^{q s s a}-\left(1-n_{t r}^{q s s a}\right)\right)\left(\ln \frac{p_{\varepsilon} n_{t r}^{q s s a}}{p_{0} \mu_{p}}-\ln \left(1-n_{t r}^{q s s a}\right)\right) d x \\
& \quad=-\int_{\Omega} R_{p} \ln \left(\frac{p n_{t r}^{q s s a}}{p_{0} \mu_{p}\left(1-n_{t r}^{q s s a}\right)}\right) d x
\end{aligned}
$$

and therefore, $P\left(n_{\varepsilon}, p_{\varepsilon}, n_{t r, \varepsilon}\right) \rightarrow P\left(n, p, n_{t r}^{q s s a}\right)$ for $\varepsilon \rightarrow 0$.

Proof of Theorem 1.3 We only have to check that the assumptions on the finiteness of the entropy $E_{0}$ and the entropy production functionals $P_{0}$ and $P$ within Theorem 2 are satisfied. The claim of this theorem then follows from the same arguments as in the proof of Theorem 1.3.

Due to the uniform $L^{\infty}$-bounds (59) of $n(t)$ and $p(t)$ for all $t \geq 0$, we know that $E_{0}(n, p)<\infty$ for all $t \geq 0$. Similarly, we deduce that $P\left(n, p, n_{t r}^{q s s a}\right)$ and $P_{0}(n, p)$ are finite for all strictly positive $t>0$ since $n, p$ are bounded away from zero and $n_{t r}^{q s s a}$ is bounded away from zero and one uniformly in $\Omega$.

Finally, the lower bounds (60) guarantee similar to Theorem 1.5 that solutions satisfy the weak entropy production law (65) for all $t_{0}>0$.

\section{Conclusion}

We have investigated the drift-diffusion-recombination system (1) modelling the transport, generation and annihilation of negatively charged electrons and positively charged holes (vacancies of electrons) in certain types of semiconductors. As depicted in Fig. 1, we have considered a two-level system augmented by an additional intermediate energy level, the so-called trap level, which results from the presence of foreign atoms inside the crystal of the semiconductor. We have derived an entropy-entropy production (EEP) inequality (cf. Theorem 1.5) which bounds the entropy functional (5) from above in terms of the entropy production functional (7). This EEP-inequality has then be used to show that the concentrations of electrons and holes converge to their equilibrium distributions at an exponential rate as time tends to infinity (cf. Theorem 1.3).

A novel achievement of our studies is the fact that the entropy method has been applied uniformly in a small time-related parameter. More precisely, the constant $C_{\text {EEP }}$ in Theorem 1.5 is independent of the lifetime $\varepsilon$ of electrons on the trap level (cf. (1)) provided $\varepsilon \in\left(0, \varepsilon_{0}\right]$ for some $\varepsilon_{0}>0$. The $\varepsilon$-independence of $C_{\mathrm{EEP}}$ transfers to the constants appearing in the exponential decay estimate in Theorem 1.3. This proves that the exponential convergence rate is independent of a quasi-steady-state 
approximation of the electrons on the trap level, which leads to the famous Shockley-Read-Hall recombination model [16, 21].

In particular, we were able to derive an EEP-inequality and the convergence estimate for the limiting Shockley-Read-Hall model. This fact is notable from a conceptual point of view as we transfer the results for $\varepsilon>0$ to the case $\varepsilon=0$ by performing the limit $\varepsilon \rightarrow 0$. We believe that our limiting approach to the Shockley-Read-Hall model may serve as an example for possible applications of this technique to fast-reaction limits and quasi-steady-state approximations.

In view of the technicalities of the proofs and the resulting length of the current paper, our results are still limited by not taking into account the self-consistent potential generated by electrons and holes, which is required by a physically more precise model. However, this leads to an additional coupling of (1) to Poisson's equation and a further increase in complexity of the problem. We expect however to resolve these issues in a future work by combining techniques and results presented in the current paper with ideas in [9], which considered a self-consistent Shockley-Read-Hall model without trapped states.

Acknowledgements Open access funding provided by Austrian Science Fund (FWF).

Funding The second author has been supported by the International Research Training Group IGDK 1754 "Optimization and Numerical Analysis for Partial Differential Equations with Nonsmooth Structures", funded by the German Research Council (DFG) and the Austrian Science Fund (FWF) under grant number [W 1244-N18].

\section{Compliance with ethical standards}

Conflict of interest Author Klemens Fellner declares that he has no conflict of interest. Author Michael Kniely declares that he has no conflict of interest.

Ethical approval This article does not contain any studies with human participants or animals performed by any of the authors.

Open Access This article is licensed under a Creative Commons Attribution 4.0 International License, which permits use, sharing, adaptation, distribution and reproduction in any medium or format, as long as you give appropriate credit to the original author(s) and the source, provide a link to the Creative Commons licence, and indicate if changes were made. The images or other third party material in this article are included in the article's Creative Commons licence, unless indicated otherwise in a credit line to the material. If material is not included in the article's Creative Commons licence and your intended use is not permitted by statutory regulation or exceeds the permitted use, you will need to obtain permission directly from the copyright holder. To view a copy of this licence, visit http://creativecommons.org/licen ses/by/4.0/.

\section{Appendix: Proof of the existence theorem}

Proof of Theorem 1.1 In order to simplify the notation, we set the parameter $\tau_{n}:=\tau_{p}:=1$ and $n_{0}:=p_{0}:=1$ throughout the proof. All arguments also apply in the case of arbitrary values for $\tau_{n}, \tau_{p}, n_{0}$ and $p_{0}$. The structure of system (1) can be further simplified via introducing new variables 


$$
u:=e^{\frac{V_{n}}{2}} n, \quad v:=e^{\frac{V_{p}}{2}} p .
$$

One obtains

$$
\begin{aligned}
& \nabla u=\frac{1}{2} e^{\frac{V_{n}}{2}} \nabla V_{n} n+e^{\frac{V_{n}}{2}} \nabla n \quad \text { and } \\
& \Delta u=e^{\frac{V_{n}}{2}}\left(\Delta n+\nabla n \cdot \nabla V_{n}+\frac{1}{4} n\left|\nabla V_{n}\right|^{2}+\frac{1}{2} n \Delta V_{n}\right)
\end{aligned}
$$

which results in

$$
\begin{aligned}
\partial_{t} u & =e^{\frac{V_{n}}{2}} \partial_{t} n=e^{\frac{V_{n}}{2}}\left(\Delta n+\nabla n \cdot \nabla V_{n}+n \Delta V_{n}+R_{n}\right) \\
& =\Delta u-e^{\frac{V_{n}}{2}}\left(\frac{1}{4} n\left|\nabla V_{n}\right|^{2}-\frac{1}{2} n \Delta V_{n}\right)+e^{\frac{V_{n}}{2}} R_{n} \\
& =\Delta u+\left(\frac{1}{2} \Delta V_{n}-\frac{1}{4}\left|\nabla V_{n}\right|^{2}\right) u+e^{\frac{V_{n}}{2}} n_{t r}-e^{V_{n}} u\left(1-n_{t r}\right) .
\end{aligned}
$$

Analogously, we derive

$$
\partial_{t} v=\Delta v+\left(\frac{1}{2} \Delta V_{p}-\frac{1}{4}\left|\nabla V_{p}\right|^{2}\right) v+e^{\frac{V_{p}}{2}}\left(1-n_{t r}\right)-e^{V_{p}} v n_{t r} .
$$

For convenience, we also introduce the abbreviations

$$
A_{n}:=\frac{1}{2} \Delta V_{n}-\frac{1}{4}\left|\nabla V_{n}\right|^{2} \in L^{\infty}(\Omega), \quad A_{p}:=\frac{1}{2} \Delta V_{p}-\frac{1}{4}\left|\nabla V_{p}\right|^{2} \in L^{\infty}(\Omega)
$$

as well as $\alpha, \beta>0$ such that the following estimates hold true a.e. in $\Omega$ :

$$
\left|A_{n}\right|,\left|A_{p}\right| \leq \alpha \quad \text { and } \quad e^{\frac{V_{n}}{2}}, e^{\frac{V_{p}}{2}}, e^{V_{n}}, e^{V_{p}} \leq \beta
$$

Next, we introduce the new variable

$$
n_{t r}^{\prime}:=1-n_{t r}
$$

for reasons of symmetry. In fact, we can prove the positivity of $n_{t r}^{\prime}$ in the same way as for $n_{t r}$, which then implies the desired bound $0 \leq n_{t r} \leq 1$. A further ingredient for establishing the positivity of the variables $u, v, n_{t r}$ and $n_{t r}^{\prime}$ is to project them onto $[0, \infty)$ and $[0,1]$, respectively, on the right hand side of the PDE-system. In this context, we use $X^{+}:=\max (X, 0)$ to denote the positive part of an arbitrary function $X$ and $X^{[0,1]}:=\min (\max (X, 0), 1)$ for the projection of $X$ to the interval $[0,1]$. The modified system now reads

$$
\left\{\begin{aligned}
\partial_{t} u-\Delta u & =A_{n} u^{+}+e^{\frac{V_{n}}{2}} n_{t r}^{[0,1]}-e^{V_{n}} u^{+} n_{t r}^{\prime[0,1]} \\
\partial_{t} v-\Delta v & =A_{p} v^{+}+e^{\frac{V_{p}}{2}} n_{t r}^{\prime[0,1]}-e^{V_{p}} v^{+} n_{t r}^{[0,1]} \\
\varepsilon \partial_{t} n_{t r} & =n_{t r}^{[0,1]}-e^{\frac{V_{p}}{2}} v^{+} n_{t r}^{[0,1]}-n_{t r}^{[0,1]}+e^{\frac{V_{n}}{2}} u^{+} n_{t r}^{\prime[0,1]} \\
\varepsilon \partial_{t} n_{t r}^{\prime} & =n_{t r}^{[0,1]}-e^{\frac{V_{n}}{2}} u^{+} n_{t r}^{\prime[0,1]}-n_{t r}^{\prime[0,1]}+e^{\frac{V_{p}}{2}} v^{+} n_{t r}^{[0,1]}
\end{aligned}\right.
$$


The no-flux boundary conditions of (1) transfer to similar conditions on $u$ and $v$. In detail, we have

$$
e^{-\frac{V_{n}}{2}} \nabla u=\nabla n+\frac{1}{2} n \nabla V_{n}
$$

and, hence,

$$
\nabla n+n \nabla V_{n}=e^{-\frac{V_{n}}{2}}\left(\nabla u+\frac{1}{2} u \nabla V_{n}\right)
$$

Therefore, the corresponding boundary conditions for $u$ and $v$ read

$$
\hat{n} \cdot\left(\nabla u+\frac{1}{2} u \nabla V_{n}\right)=\hat{n} \cdot\left(\nabla v+\frac{1}{2} v \nabla V_{p}\right)=0 .
$$

Furthermore, we assume that the corresponding initial states satisfy

$$
\left(u_{I}, v_{I}, n_{t r, I}, n_{t r, I}^{\prime}\right) \in L_{+}^{\infty}(\Omega)^{4}, \quad n_{t r, I}+n_{t r, I}^{\prime}=1 .
$$

In this situation, $\left\|n_{t r, I}\right\|_{L^{\infty}(\Omega)}+\left\|n_{t r, I}^{\prime}\right\|_{L^{\infty}(\Omega)} \geq 1$ and we set

$$
I:=\left\|u_{I}\right\|_{L^{\infty}(\Omega)}+\left\|v_{I}\right\|_{L^{\infty}(\Omega)}+\left\|n_{t r, I}\right\|_{L^{\infty}(\Omega)}+\left\|n_{t r, I}^{\prime}\right\|_{L^{\infty}(\Omega)} \geq 1 .
$$

We now aim to apply Banach's fixed-point theorem to obtain a solution of (74)-(76).

Step 1: Definition of the fixed-point iteration. For any time $T>0$ (to be chosen sufficiently small in the course of the fixed-point argument), we introduce the space

$$
X_{T}:=C\left([0, T], L^{2}(\Omega)\right)^{4}
$$

and the closed subspace

$$
\begin{aligned}
M_{T}:=\{ & \left(u, v, n_{t r}, n_{t r}^{\prime}\right) \in X_{T} \mid\left(u(0), v(0), n_{t r}(0), n_{t r}^{\prime}(0)\right)=\left(u_{I}, v_{I}, n_{t r, I}, n_{t r, I}^{\prime}\right), \\
& \max _{0 \leq t \leq T}\|u(t)\|_{L^{2}(\Omega)}, \max _{0 \leq t \leq T}\|v(t)\|_{L^{2}(\Omega)}, \max _{0 \leq t \leq T}\left\|n_{t r}(t)\right\|_{L^{2}(\Omega)}, \\
& \left.\max _{0 \leq t \leq T}\left\|n_{t r}^{\prime}(t)\right\|_{L^{2}(\Omega)},\|u\|_{L^{\infty}((0, T) \times \Omega)},\|v\|_{L^{\infty}((0, T) \times \Omega)} \leq 2 I\right\} \subset X_{T} .
\end{aligned}
$$

The fixed-point mapping $\mathcal{S}: X_{T} \rightarrow X_{T}$ is now defined via

$$
\mathcal{S}\left(\widetilde{u}, \widetilde{v}, \tilde{n}_{t r}, \tilde{n}_{t r}^{\prime}\right):=\left(u, v, n_{t r}, n_{t r}^{\prime}\right)
$$

where $\left(u, v, n_{t r}, n_{t r}^{\prime}\right)$ is the solution of the following PDE-system subject to the boundary and initial conditions specified above:

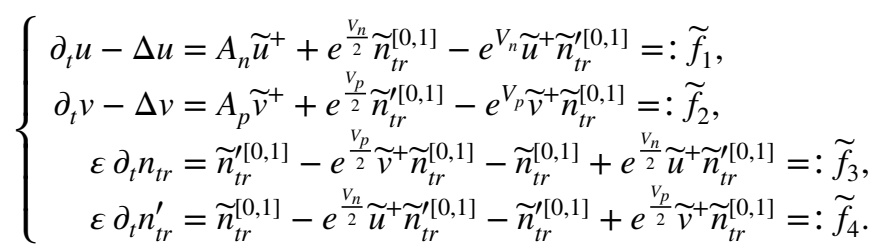


We first show that $\left(u, v, n_{t r}, n_{t r}^{\prime}\right)=\mathcal{S}\left(\widetilde{u}, \widetilde{v}, \tilde{n}_{t r}, \tilde{n}_{t r}^{\prime}\right) \in X_{T}$ provided $\left(\tilde{u}, \widetilde{v}, \tilde{n}_{t r}, \tilde{n}_{t r}^{\prime}\right) \in X_{T}$. Due to $\widetilde{f}_{1}, \widetilde{f}_{2} \in L^{2}((0, T) \times \Omega)$, it is known from classical PDE-theory (see e.g. [3]) that

$$
\begin{aligned}
u, v \in W_{2}(0, T)= & \left\{f \in L^{2}\left((0, T), H^{1}(\Omega)\right) \mid \partial_{t} f \in L^{2}\left((0, T), H^{1}(\Omega)^{*}\right)\right\} \\
& \hookrightarrow C\left([0, T], L^{2}(\Omega)\right) .
\end{aligned}
$$

And since

$$
n_{t r}(t)=n_{t r}(0)+\frac{1}{\varepsilon} \int_{0}^{t} \widetilde{f}_{3}(s) d s
$$

for almost all $t \in[0, T]$, we deduce

$$
\begin{aligned}
\left\|n_{t r}(t)\right\|_{L^{2}(\Omega)} & \leq\left\|n_{t r}(0)\right\|_{L^{2}(\Omega)}+\frac{1}{\varepsilon} \int_{0}^{t}\left\|\tilde{f}_{3}(s)\right\|_{L^{2}(\Omega)} d s \\
& \leq I+\frac{T}{\varepsilon} \max _{0 \leq t \leq T}\left\|\tilde{f}_{3}(s)\right\|_{L^{2}(\Omega)} .
\end{aligned}
$$

Hence, $n_{t r} \in L^{\infty}\left((0, T), L^{2}(\Omega)\right)$. Moreover, we derive for $[0, T] \ni t_{k} \rightarrow t \in[0, T]$,

$$
\begin{aligned}
\left\|n_{t r}\left(t_{k}\right)-n_{t r}(t)\right\|_{L^{2}(\Omega)} & \leq \frac{1}{\epsilon}\left|\int_{t}^{t_{k}}\left\|\tilde{f}_{3}(s)\right\|_{L^{2}(\Omega)} d s\right| \\
& \leq \frac{\left|t_{k}-t\right|}{\epsilon} \max _{0 \leq t \leq T}\left\|\tilde{f}_{3}(s)\right\|_{L^{2}(\Omega)} \stackrel{k \rightarrow \infty}{\longrightarrow} 0 .
\end{aligned}
$$

This proves $n_{t r} \in C\left([0, T], L^{2}(\Omega)\right)$. The same arguments can be applied to $n_{t r}^{\prime}$.

Step 2: Invariance of $M_{T}$. Now, let $\left(\tilde{u}, \widetilde{v}, \widetilde{n}_{t r}, \widetilde{n}_{t r}^{\prime}\right) \in M_{T}$. Similar to the strategy of e.g. $[1,15,26]$, we perform the subsequent calculations for any $q \in 2 \mathbb{N}_{+}$and every $t \in[0, T]:$

$$
\begin{aligned}
\int_{0}^{t} \frac{d}{d s} \int_{\Omega} \frac{u^{q}}{q} d x d s= & \int_{0}^{t} \int_{\Omega} u^{q-1} \partial_{t} u d x d s=\int_{0}^{t} \int_{\Omega} u^{q-1} \Delta u d x d s \\
& +\int_{0}^{t} \int_{\Omega} u^{q-1} \widetilde{f}_{1} d x d s \leq-(q-1) \int_{0}^{t} \int_{\Omega} u^{q-2}|\nabla u|^{2} d x d s \\
& -\frac{1}{2} \int_{0}^{t} \int_{\partial \Omega} u^{q} \hat{n} \cdot \nabla V_{n} d \sigma d s+\left\|\tilde{f}_{1}\right\|_{L^{\infty}((0, T) \times \Omega)} \int_{0}^{t} \int_{\Omega}|u|^{q-1} d x d s \\
\leq & (2 \alpha I+\beta+2 \beta I) \int_{0}^{t}\|u\|_{L^{q}(\Omega)}^{q-1} d s .
\end{aligned}
$$

Note that the last term in the second and the first term in the third line are both non-positive due to $q \in 2 \mathbb{N}$ and assumption (4). Introducing $\gamma:=2 \alpha I+\beta+2 \beta I$, we obtain

$$
\|u(t)\|_{L^{q}(\Omega)}^{q}-\|u(0)\|_{L^{q}(\Omega)}^{q} \leq q \gamma \int_{0}^{t}\|u(s)\|_{L^{q}(\Omega)}^{q-1} d s .
$$


This inequality already implies a linear bound on the $L^{\infty}$-norm of $u$ as we shall see below (cf. [2]). We define

$$
U(t):=q \gamma \int_{0}^{t}\|u(s)\|_{L^{q}(\Omega)}^{q-1} d s
$$

and note that $U(0)=0$. Estimate $(78)$ entails

$$
U^{\prime}(t)=q \gamma\left(\|u(t)\|_{L^{q}(\Omega)}^{q}\right)^{\frac{q-1}{q}} \leq q \gamma\left(\eta+\|u(0)\|_{L^{q}(\Omega)}^{q}+U(t)\right)^{\frac{q-1}{q}}
$$

for all $t \in[0, T]$, where $\eta>0$ is an arbitrary constant, which guarantees that the expression $X:=\eta+\|u(0)\|_{L^{q}(\Omega)}^{q}+U(t)$ is strictly positive. Multiplying both sides with $X^{(1-q) / q}$ and integrating from 0 to $t$ gives

$$
\int_{0}^{t}\left(\eta+\|u(0)\|_{L^{q}(\Omega)}^{q}+U(s)\right)^{\frac{1-q}{q}} U^{\prime}(s) d s \leq \int_{0}^{t} q \gamma d s .
$$

We now substitute $\sigma:=U(s)$ and deduce

$$
\begin{aligned}
q \gamma t & \geq \int_{0}^{U(t)}\left(\eta+\|u(0)\|_{L^{q}(\Omega)}^{q}+\sigma\right)^{\frac{1}{q}-1} d \sigma=\left.q\left(\eta+\|u(0)\|_{L^{q}(\Omega)}^{q}+\sigma\right)^{\frac{1}{q}}\right|_{0} ^{U(t)} \\
& =q\left(\eta+\|u(0)\|_{L^{q}(\Omega)}^{q}+U(t)\right)^{\frac{1}{q}}-q\left(\eta+\|u(0)\|_{L^{q}(\Omega)}^{q}\right)^{\frac{1}{q}} \\
& \geq q\left(\|u(t)\|_{L^{q}(\Omega)}^{q}\right)^{\frac{1}{q}}-q\left(\eta+\|u(0)\|_{L^{q}(\Omega)}^{q}\right)^{\frac{1}{q}}
\end{aligned}
$$

where we have used (78) in the last step. Therefore,

$$
\|u(t)\|_{L^{q}(\Omega)} \leq\left(\eta+\|u(0)\|_{L^{q}(\Omega)}^{q}\right)^{\frac{1}{q}}+\gamma t
$$

and, taking the limit $\eta \rightarrow 0$,

$$
\|u(t)\|_{L^{q}(\Omega)} \leq\|u(0)\|_{L^{q}(\Omega)}+\gamma t \leq I+\gamma t .
$$

As the bound on the right hand side is independent of $q$, we even obtain

$$
\|u(t)\|_{L^{\infty}(\Omega)} \leq I+\gamma t,
$$

for all $t \in[0, T]$. This result naturally gives rise to

$$
\|u\|_{L^{\infty}((0, T) \times \Omega)} \leq I+\gamma T .
$$

An analogous estimate is valid for $v$. As a result, we obtain

$$
\|u\|_{L^{\infty}((0, T) \times \Omega)},\|v\|_{L^{\infty}((0, T) \times \Omega)} \leq 2 I
$$

for $T>0$ chosen sufficiently small.

Employing (79), we also derive 


$$
\max _{0 \leq t \leq T}\|u(t)\|_{L^{2}(\Omega)} \leq \max _{0 \leq t \leq T}\|u(t)\|_{L^{\infty}(\Omega)} \leq I+\gamma T .
$$

The same argument is applicable to $v$, which results in

$$
\max _{0 \leq t \leq T}\|u(t)\|_{L^{2}(\Omega)}, \max _{0 \leq t \leq T}\|v(t)\|_{L^{2}(\Omega)} \leq 2 I
$$

for sufficiently small $T>0$. The corresponding bounds on $n_{t r}$ and $n_{t r}^{\prime}$ can be deduced from the formula

$$
n_{t r}(t)=n_{t r}(0)+\frac{1}{\varepsilon} \int_{0}^{t} \widetilde{f}_{3}(s) d s
$$

and from an analogous one for $n_{t r}^{\prime}$. In fact,

$$
\left\|n_{t r}(t)\right\|_{L^{2}(\Omega)} \leq\left\|n_{t r}(0)\right\|_{L^{2}(\Omega)}+\frac{1}{\varepsilon} \int_{0}^{t}\left\|\tilde{f}_{3}(s)\right\|_{L^{2}(\Omega)} d s \leq I+\frac{T}{\varepsilon}(2+4 \beta I)
$$

and, hence,

$$
\max _{0 \leq t \leq T}\left\|n_{t r}(t)\right\|_{L^{2}(\Omega)}, \max _{0 \leq t \leq T}\left\|n_{t r}^{\prime}(t)\right\|_{L^{2}(\Omega)} \leq 2 I
$$

for $T>0$ sufficiently small.

Step 3: Contraction property of $\mathcal{S}$. We consider $\left(\widetilde{u}_{1}, \widetilde{v}_{1}, \tilde{n}_{t r, 1}, \tilde{n}_{t r, 1}^{\prime}\right),\left(\widetilde{u}_{2}, \widetilde{v}_{2}, \tilde{n}_{t r, 2}, \tilde{n}_{t r, 2}^{\prime}\right) \in M_{T} \quad$ and $\quad$ the corresponding solutions $\quad\left(u_{1}, v_{1}, n_{t r, 1}, n_{t r, 1}^{\prime}\right)=\mathcal{S}\left(\widetilde{u}_{1}, \widetilde{v}_{1}, \tilde{n}_{t r, 1}, \widetilde{n}_{t r, 1}^{\prime}\right) \in M_{T} \quad$ and $\left(u_{2}, v_{2}, n_{t r, 2}, n_{t r, 2}^{\prime}\right)=\mathcal{S}\left(\widetilde{u}_{2}, \widetilde{v}_{2}, \widetilde{n}_{t r, 2}, \widetilde{n}_{t r, 2}^{\prime}\right) \in \stackrel{M}{T}_{T}$. We further introduce the notation

$$
u:=u_{1}-u_{2}, \quad \widetilde{u}:=\widetilde{u}_{1}-\widetilde{u}_{2}
$$

and similarly $v, n_{t r}, n_{t r}^{\prime}, \widetilde{v}, \tilde{n}_{t r}$ and $\tilde{n}_{t r}^{\prime}$. Then, we have to show that

$$
\left\|\left(u, v, n_{t r}, n_{t r}^{\prime}\right)\right\|_{X_{T}} \leq c\left\|\left(\widetilde{u}, \widetilde{v}, \tilde{n}_{t r}, \tilde{n}_{t r}^{\prime}\right)\right\|_{X_{T}}
$$

with a constant $c \in(0,1)$ on a time interval $[0, T]$ small enough. The norm in $X_{T}$ is defined as

$$
\begin{aligned}
\left\|\left(u, v, n_{t r}, n_{t r}^{\prime}\right)\right\|_{X_{T}}:= & \max _{0 \leq t \leq T}\|u(t)\|_{L^{2}(\Omega)}+\max _{0 \leq t \leq T}\|v(t)\|_{L^{2}(\Omega)} \\
& +\max _{0 \leq t \leq T}\left\|n_{t r}(t)\right\|_{L^{2}(\Omega)}+\max _{0 \leq t \leq T}\left\|n_{t r}^{\prime}(t)\right\|_{L^{2}(\Omega)} .
\end{aligned}
$$

We obtain the following system by taking the difference of corresponding equations of the system for the 1- and the 2-variables, respectively: 


$$
\left\{\begin{aligned}
\partial_{t} u-\Delta u= & A_{n}\left(\widetilde{u}_{1}^{+}-\widetilde{u}_{2}^{+}\right)+e^{\frac{V_{n}}{2}}\left(\widetilde{n}_{t r, 1}^{[0,1]}-\widetilde{n}_{t r, 2}^{[0,1]}\right) \\
& -e^{V_{n}}\left(\widetilde{u}_{1}^{+} \widetilde{n}_{t r, 1}^{\prime[0,1]}-\widetilde{u}_{2}^{+} \widetilde{n}_{t r, 2}^{\prime[0,1]}\right)=: \widetilde{f}_{1}, \\
\partial_{t} v-\Delta v= & A_{p}\left(\widetilde{v}_{1}^{+}-\widetilde{v}_{2}^{+}\right)+e^{\frac{V_{p}}{2}}\left(\widetilde{n}_{t r, 1}^{\prime[0,1]}-\widetilde{n}_{t r, 2}^{[0,1]}\right) \\
& -e^{V_{p}}\left(\widetilde{v}_{1}^{+} \widetilde{n}_{t r, 1}^{[0,1]}-\widetilde{v}_{2}^{+} \widetilde{n}_{t r, 2}^{[0,1]}\right)=: \widetilde{f}_{2}, \\
\varepsilon \partial_{t} n_{t r}= & \widetilde{n}_{t r, 1}^{\prime[0,1]}-\widetilde{n}_{t r, 2}^{\prime[0,1]}-e^{\frac{V_{p}}{2}}\left(\widetilde{v}_{1}^{+} \widetilde{n}_{t r, 1}^{[0,1]}-\widetilde{v}_{2}^{+} \widetilde{n}_{t r, 2}^{[0,1]}\right) \\
& -\widetilde{n}_{t r, 1}^{[0,1]}+\widetilde{n}_{t r, 2}^{[0,1]}+e^{\frac{V_{n}}{2}}\left(\widetilde{u}_{1}^{+} \widetilde{n}_{t r, 1}^{\prime[0,1]}-\widetilde{u}_{2}^{+} \widetilde{n}_{t r, 2}^{[0,1]}\right)=: \widetilde{f}_{3}, \\
\varepsilon \partial_{t} n_{t r}^{\prime}= & \widetilde{n}_{t r, 1}^{[0,1]}-\widetilde{n}_{t r, 2}^{[0,1]}-e^{\frac{V_{n}}{2}}\left(\widetilde{u}_{1}^{+} \widetilde{n}_{t r, 1}^{\prime[0,1]}-\widetilde{u}_{2}^{+} \widetilde{n}_{t r, 2}^{\prime[0,1]}\right) \\
& -\widetilde{n}_{t r, 1}^{[0,1]}+\widetilde{n}_{t r, 2}^{\prime[0,1]}+e^{\frac{V_{p}}{2}}\left(\widetilde{v}_{1}^{+} \widetilde{n}_{t r, 1}^{[0,1]}-\widetilde{v}_{2}^{+} \widetilde{n}_{t r, 2}^{[0,1]}\right)=: \widetilde{f}_{4} .
\end{aligned}\right.
$$

We mention that $u$ and $v$ are subject to the boundary conditions

$$
\hat{n} \cdot\left(\nabla u+\frac{1}{2} u \nabla V_{n}\right)=\hat{n} \cdot\left(\nabla v+\frac{1}{2} v \nabla V_{p}\right)=0
$$

and the homogeneous initial conditions

$$
u(0)=v(0)=n_{t r}(0)=n_{t r}^{\prime}(0)=0 .
$$

First, one finds

$$
\max _{0 \leq t \leq T}\|u(t)\|_{L^{2}(\Omega)} \leq C_{1}\|u\|_{W_{2}(0, T)} \leq C_{1} C_{2}\left\|\tilde{f}_{1}\right\|_{L^{2}((0, T) \times \Omega)}
$$

where $C_{1}>0$ is the constant resulting from the embedding $W_{2}(0, T) \hookrightarrow C\left([0, T], L^{2}(\Omega)\right)$. The constant $C_{2}>0$ originates from well-known parabolic regularity estimates for $\|u\|_{W_{2}(0, T)}$ in terms of the $L^{2}$-norms of $\widetilde{f}_{1}$ and $u(0)=0$. Therefore,

$$
\begin{aligned}
\max _{0 \leq t \leq T}\|u(t)\|_{L^{2}(\Omega)} \leq & C_{1} C_{2}\left(\alpha\left\|\tilde{u}_{1}^{+}-\widetilde{u}_{2}^{+}\right\|_{L^{2}((0, T) \times \Omega)}+\beta\left\|\tilde{n}_{t r, 1}^{[0,1]}-\widetilde{n}_{t r, 2}^{[0,1]}\right\|_{L^{2}((0, T) \times \Omega)}\right. \\
& +\beta\left\|\tilde{u}_{1}^{+}-\widetilde{u}_{2}^{+}\right\|_{L^{2}((0, T) \times \Omega)}\left\|\widetilde{n}_{t r, 1}^{\prime[0,1]}\right\|_{L^{\infty}((0, T) \times \Omega)} \\
& \left.+\beta\left\|\tilde{u}_{2}^{+}\right\|_{L^{\infty}((0, T) \times \Omega)}\left\|\widetilde{n}_{t r, 1}^{[0,1]}-\widetilde{n}_{t r, 2}^{[0,1]}\right\|_{L^{2}((0, T) \times \Omega)}\right) \\
\leq & C_{1} C_{2}\left(\beta\left\|\tilde{n}_{t r}\right\|_{L^{2}((0, T) \times \Omega)}+(\alpha+\beta)\|\tilde{u}\|_{L^{2}((0, T) \times \Omega)}\right. \\
& +2 \beta I\left\|\tilde{n}_{t r}^{\prime}\right\|_{L^{2}((0, T) \times \Omega)} .
\end{aligned}
$$

Moreover, every $f \in C\left([0, T], L^{2}(\Omega)\right)$ fulfils

$$
\|f\|_{L^{2}((0, T) \times \Omega)}^{2}=\int_{0}^{T} \int_{\Omega} f^{2} d x d t \leq \int_{0}^{T} d t \max _{0 \leq t \leq T}\|f(t)\|_{L^{2}(\Omega)}^{2}=T\|f\|_{C\left([0, T], L^{2}(\Omega)\right)}^{2}
$$

and we proceed with the previous estimates to derive 


$$
\begin{aligned}
\max _{0 \leq t \leq T}\|u(t)\|_{L^{2}(\Omega)} \leq & C_{1} C_{2}(\alpha+2 \beta I) \sqrt{T}\left(\left\|\widetilde{n}_{t r}\right\|_{C\left([0, T], L^{2}(\Omega)\right)}\right. \\
& \left.+\|\widetilde{u}\|_{C\left([0, T], L^{2}(\Omega)\right)}+\left\|\widetilde{n}_{t r}^{\prime}\right\|_{C\left([0, T], L^{2}(\Omega)\right)}\right) .
\end{aligned}
$$

In a similar way, we arrive at

$$
\begin{aligned}
\max _{0 \leq t \leq T}\|v(t)\|_{L^{2}(\Omega)} \leq & C_{1} C_{2}(\alpha+2 \beta I) \sqrt{T}\left(\left\|\tilde{n}_{t r}^{\prime}\right\|_{C\left([0, T], L^{2}(\Omega)\right)}\right. \\
& \left.+\|\widetilde{v}\|_{C\left([0, T], L^{2}(\Omega)\right)}+\left\|\tilde{n}_{t r}\right\|_{C\left([0, T], L^{2}(\Omega)\right)}\right) .
\end{aligned}
$$

Due to $n_{t r}(0)=0$, one obtains

$$
n_{t r}(t)=\frac{1}{\varepsilon} \int_{0}^{t} \widetilde{f}_{3} d s
$$

for $t \in[0, T]$ and, using similar techniques as above,

$$
\begin{aligned}
\max _{0 \leq t \leq T}\left\|n_{t r}(t)\right\|_{L^{2}(\Omega)} \leq & \frac{1}{\varepsilon} \int_{0}^{T}\left\|\tilde{f}_{3}\right\|_{L^{2}(\Omega)} d s \leq \frac{\sqrt{T}}{\varepsilon}\left\|\tilde{f}_{3}\right\|_{L^{2}((0, T) \times \Omega)} \\
\leq & \frac{1+2 \beta I}{\varepsilon} \sqrt{T}\left(\|\widetilde{u}\|_{L^{2}((0, T) \times \Omega)}+\|\widetilde{v}\|_{L^{2}((0, T) \times \Omega)}\right. \\
& \left.+\left\|\tilde{n}_{t r}\right\|_{L^{2}((0, T) \times \Omega)}+\left\|\tilde{n}_{t r}^{\prime}\right\|_{L^{2}((0, T) \times \Omega)}\right) \\
\leq & \frac{1+2 \beta I}{\varepsilon} T\left(\|\widetilde{u}\|_{C\left([0, T], L^{2}(\Omega)\right)}+\|\widetilde{v}\|_{C\left([0, T], L^{2}(\Omega)\right)}\right. \\
& \left.+\left\|\tilde{n}_{t r}\right\|_{C\left([0, T], L^{2}(\Omega)\right)}+\left\|\tilde{n}_{t r}^{\prime}\right\|_{C\left([0, T], L^{2}(\Omega)\right)}\right) .
\end{aligned}
$$

Note that because of $\tilde{f}_{4}=-\widetilde{f}_{3}$, the last estimate equally serves as an upper bound for $\left\|n_{t r}^{\prime}(t)\right\|_{L^{2}(\Omega)}$. Taking the sum of the above estimates and choosing $T>0$ sufficiently small yields

$$
\left\|\left(u, v, n_{t r}, n_{t r}^{\prime}\right)\right\|_{X_{T}} \leq c\left\|\left(\widetilde{u}, \widetilde{v}, \tilde{n}_{t r}, \tilde{n}_{t r}^{\prime}\right)\right\|_{X_{T}}
$$

with some $c \in(0,1)$.

Step 4: Solution of (1). Step 2 and Step 3 imply that for $T>0$ sufficiently small the mapping $\mathcal{S}: M_{T} \rightarrow M_{T}$ is a contraction. Banach's fixed point theorem, thus, guarantees that there exists a unique $\left(u, v, n_{t r}, n_{t r}^{\prime}\right) \in M_{T}$ such that $\mathcal{S}\left(u, v, n_{t r}, n_{t r}^{\prime}\right)=\left(u, v, n_{t r}, n_{t r}^{\prime}\right)$. Moreover, due to standard parabolic regularity for $(u, v)$, the fixed-point $\left(u, v, n_{t r}, n_{t r}^{\prime}\right)$ is the unique weak solution of 


$$
\left\{\begin{aligned}
\partial_{t} u-\Delta u & =A_{n} u^{+}+e^{\frac{V_{n}}{2}} n_{t r}^{[0,1]}-e^{V_{n}} u^{+} n_{t r}^{\prime[0,1]}, \\
\partial_{t} v-\Delta v & =A_{p} v^{+}+e^{\frac{V_{p}}{2}} n_{t r}^{\prime[0,1]}-e^{V_{p}} v^{+} n_{t r}^{[0,1]}, \\
\varepsilon \partial_{t} n_{t r} & =n_{t r}^{\prime[0,1]}-e^{\frac{V_{p}}{2}} v^{+} n_{t r}^{[0,1]}-n_{t r}^{[0,1]}+e^{\frac{V_{n}}{2}} u^{+} n_{t r}^{[0,1]}, \\
\varepsilon \partial_{t} n_{t r}^{\prime} & =n_{t r}^{[0,1]}-e^{\frac{V_{n}}{2}} u^{+} n_{t r}^{\prime[0,1]}-n_{t r}^{\prime[0,1]}+e^{\frac{V_{p}}{2}} v^{+} n_{t r}^{[0,1]}
\end{aligned}\right.
$$

In order to prove the non-negativity of $u, v, n_{t r}$ and $n_{t r}^{\prime}$, we adapt an argument from [26]. First, we define

$$
h:=\min (0, u)
$$

on $[0, T] \times \Omega$ and notice that $h \leq 0$ and $h(t=0)=0$ a.e. since $u(0) \geq 0$ a.e. We now multiply the first equation in (81) with $h$ and integrate over $(0, t) \times \Omega$ for $t \in[0, T]$. This yields

$$
\begin{aligned}
\int_{0}^{t} \int_{\Omega} \partial_{s} u h d x d s= & \int_{0}^{t} \int_{\Omega} \Delta u h d x d s+\int_{0}^{t} \int_{\Omega} A_{n} u^{+} h d x d s \\
& +\int_{0}^{t} \int_{\Omega}\left(e^{\frac{V_{n}}{2}} n_{t r}^{[0,1]}-e^{V_{n}} u^{+} n_{t r}^{\prime[0,1]}\right) h d x d s .
\end{aligned}
$$

The first term on the right hand side of (82) can be seen to be non-positive using integration by parts and the boundary condition from (75):

$$
\begin{aligned}
\int_{0}^{t} \int_{\Omega} \Delta u h d x d s & =-\int_{0}^{t} \int_{\Omega} \nabla u \cdot \nabla h d x d s-\frac{1}{2} \int_{0}^{t} \int_{\partial \Omega} u h \hat{n} \cdot \nabla V_{n} d \sigma d s \\
& \leq-\int_{0}^{t} \int_{\Omega} \nabla u \cdot \nabla h d x d s=-\int_{0}^{t} \int_{\Omega} \nabla h \cdot \nabla h d x d s \leq 0
\end{aligned}
$$

due to $u h \geq 0, \hat{n} \cdot \nabla V_{n} \geq 0$, and since $\nabla h \neq 0$ holds true only in the case $u<0$, where we have $\nabla u=\nabla h$ in $L^{2}$, see e.g. [14]. Moreover,

$$
\int_{0}^{t} \int_{\Omega} A_{n} u^{+} h d x d s=0
$$

and the third term in (82) is again non-positive as an integral over non-positive quantities:

$$
\int_{0}^{t} \int_{\Omega}\left(e^{\frac{V_{n}}{2}} n_{t r}^{[0,1]}-e^{V_{n}} u^{+} n_{t r}^{\prime[0,1]}\right) h d x d s=\int_{0}^{t} \int_{\Omega} e^{\frac{V_{n}}{2}} n_{t r}^{[0,1]} h d x d s \leq 0
$$

as a consequence of $u^{+} h=0$ in $L^{2}(\Omega)$. The left hand side of (82) can be reformulated as

$$
\int_{0}^{t} \int_{\Omega} \partial_{s} u h d x d s=\int_{0}^{t} \int_{\Omega} \partial_{s} h h d x d s=\frac{1}{2} \int_{\Omega} \int_{0}^{t}\left(\frac{d}{d s} h^{2}\right) d s d x=\frac{1}{2}\|h(t)\|_{L^{2}(\Omega)}^{2} .
$$

For the first step, we have used that the integrand $\partial_{s} u h$ only contributes to the integral if $h<0$. But in this case, $u=h$ and, hence, $\partial_{s} u=\partial_{s} h$ in $L^{2}$, see e.g. [14]. This 
proves $\|h(t)\|_{L^{2}(\Omega)} \leq 0$ for all $t \in[0, T]$, which establishes $h(t)=0$ in $L^{2}(\Omega)$ for all $t \in[0, T]$, and thus $u(t, x) \geq 0$ for all $t \in[0, T]$ and a.e. $x \in \Omega$. In the same way, one can show that $v(t, x) \geq 0$ for all $t \in[0, T]$ and a.e. $x \in \Omega$.

The non-negativity of $n_{t r}$ follows from a similar idea using

$$
h:=\min \left(0, n_{t r}\right) \text {. }
$$

Again, $h \leq 0$ and $h(t=0)=0$ due to $n_{t r}(0) \geq 0$. Multiplying the third equation of (81) with $h$ and integrating over $(0, t) \times \Omega, t \in[0, T]$, we find

$$
\varepsilon \int_{0}^{t} \int_{\Omega} \partial_{s} n_{t r} h d x d s=\int_{0}^{t} \int_{\Omega}\left(n_{t r}^{\prime[0,1]}-e^{\frac{v_{p}}{2}} v^{+} n_{t r}^{[0,1]}-n_{t r}^{[0,1]}+e^{\frac{V_{n}}{2}} u^{+} n_{t r}^{\prime[0,1]}\right) h d x d s .
$$

As before, all terms under the integral on the right hand side involving $n_{t r}^{[0,1]}$ vanish. Consequently,

$$
\frac{\varepsilon}{2}\|h(t)\|_{L^{2}(\Omega)}^{2}=\varepsilon \int_{0}^{t} \int_{\Omega} \partial_{s} h h d x d s=\int_{0}^{t} \int_{\Omega}\left(n_{t r}^{\prime[0,1]}+e^{\frac{V_{n}}{2}} u^{+} n_{t r}^{\prime[0,1]}\right) h d x d s \leq 0
$$

for all $t \in[0, T]$. The same result holds true for $n_{t r}^{\prime}$. Therefore, we have verified that $n_{t r}(t, x), n_{t r}^{\prime}(t, x) \geq 0$ for all $t \in[0, T]$ and a.e. $x \in \Omega$.

The non-negativity of $n_{t r}$ and $n_{t r}^{\prime}$ together with $n_{t r}^{\prime}=1-n_{t r}$ from (73) now even imply

$$
n_{t r}(t, x), n_{t r}^{\prime}(t, x) \in[0,1], \quad \text { for all } t \in[0, T] \text { and a.e. } \quad x \in \Omega .
$$

This allows us to identify the unique weak solution $\left(u, v, n_{t r}, n_{t r}^{\prime}\right)$ of (81) to equally solve

$$
\left\{\begin{aligned}
\partial_{t} u-\Delta u & =A_{n} u+e^{\frac{V_{n}}{2}} n_{t r}-e^{V_{n}} u\left(1-n_{t r}\right), \\
\partial_{t} v-\Delta v & =A_{p} v+e^{\frac{V_{p}}{2}}\left(1-n_{t r}\right)-e^{V_{p}} v n_{t r}, \\
\varepsilon \partial_{t} n_{t r} & =1-n_{t r}-e^{\frac{V_{p}}{2}} v n_{t r}-n_{t r}+e^{\frac{V_{n}}{2}} u\left(1-n_{t r}\right),
\end{aligned}\right.
$$

which is the transform version of the original problem (1).

$\mathrm{Up}$ to now, we have proven that there exists a unique solution $\left(u, v, n_{t r}\right) \in C\left([0, T], L^{2}(\Omega)\right)^{3}$ such that $\left(u, v, n_{t r}, 1-n_{t r}\right) \in M_{T}$ on a sufficiently small time interval $[0, T]$.

Step 5: Global solution. We now fix $T^{*}>0$ in such a way that $\left[0, T^{*}\right)$ is the maximal time interval of existence for the solution $\left(u, v, n_{t r}\right) \in C\left([0, T], L^{2}(\Omega)\right)^{3}$ of (83). Moreover, we choose some arbitrary $q \in \mathbb{N}_{\geq 2}$ and multiply the first equation in (83) with $u^{q-1}$. Integrating over $\Omega$ at time $t \in\left[0, T^{*}\right)$ gives

$$
\begin{aligned}
\frac{d}{d t} \int_{\Omega} \frac{u^{q}}{q} d x= & \int_{\Omega} u^{q-1} \partial_{t} u d x=\int_{\Omega} u^{q-1} \Delta u d x+\int_{\Omega} A_{n} u^{q} d x \\
& +\int_{\Omega} u^{q-1}\left(e^{\frac{V_{n}}{2}} n_{t r}-e^{V_{n}} u\left(1-n_{t r}\right)\right) d x
\end{aligned}
$$


Integration by parts and the estimates $\left|A_{n}\right| \leq \alpha,\left|e^{\frac{V_{n}}{2}} n_{t r}-e^{V_{n}} u\left(1-n_{t r}\right)\right| \leq \beta(1+u)$ further yield

$$
\begin{aligned}
\frac{d}{d t} \int_{\Omega} \frac{u^{q}}{q} d x \leq & -(q-1) \int_{\Omega} u^{q-2}|\nabla u|^{2} d x-\frac{1}{2} \int_{\partial \Omega} u^{q} \hat{n} \cdot \nabla V_{n} d \sigma d s \\
& +\alpha \int_{\Omega} u^{q} d x+\beta \int_{\Omega}\left(u^{q-1}+u^{q}\right) d x
\end{aligned}
$$

Moreover, we derive

$$
\int_{\Omega} u^{q-1} d x=\int_{\{u \leq 1\}} u^{q-1} d x+\int_{\{u>1\}} \frac{u^{q}}{u} d x \leq \int_{\Omega} 1 d x+\int_{\Omega} u^{q} d x=1+\int_{\Omega} u^{q} d x
$$

where we used $|\Omega|=1$. Hence,

$$
\frac{d}{d t} \int_{\Omega} \frac{u^{q}}{q} d x \leq \beta+(\alpha+2 \beta) \int_{\Omega} u^{q} d x \leq \gamma\left(1+\int_{\Omega} u^{q} d x\right)
$$

after defining $\gamma:=\alpha+2 \beta$. This results in

$$
\frac{d}{d t} \int_{\Omega} u^{q} d x \leq \gamma q\left(1+\int_{\Omega} u^{q} d x\right)
$$

which can be integrated over time from 0 to $t$ :

$$
\|u(t)\|_{L^{q}(\Omega)}^{q} \leq\|u(0)\|_{L^{q}(\Omega)}^{q}+\gamma q \int_{0}^{t}\left(1+\|u(s)\|_{L^{q}(\Omega)}^{q}\right) d s .
$$

From this generalised Gronwall-type inequality, we deduce (cf. [2])

$$
\|u(t)\|_{L^{q}(\Omega)}^{q} \leq\|u(0)\|_{L^{q}(\Omega)}^{q} e^{\gamma q t}+e^{\gamma q t}-1<\left(1+\|u(0)\|_{L^{q}(\Omega)}^{q}\right) e^{\gamma q t}
$$

and

$$
\|u(t)\|_{L^{q}(\Omega)} \leq\left(1+\|u(0)\|_{L^{q}(\Omega)}\right) e^{\gamma t} \leq I e^{\gamma t}
$$

since $1+\|u(0)\|_{L^{q}(\Omega)} \leq 1+\|u(0)\|_{L^{\infty}(\Omega)} \leq I$. As $I e^{\gamma t}$ is independent of $q$, we even arrive at

$$
\|u(t)\|_{L^{\infty}(\Omega)} \leq I e^{\gamma t} .
$$

In the same way, we can show that $\|v(t)\|_{L^{\infty}(\Omega)} \leq I e^{\gamma t}$ for all $t \in\left[0, T^{*}\right)$. As a consequence, we obtain that the solution $\left(u, v, n_{t r}\right) \in C\left([0, T], L^{2}(\Omega)\right)^{3}$ can be extended for all times, i.e. $T^{*}=\infty$.

Step 6: $L^{\infty}$-bounds for $n$ and $p$. We now prove the linearly growing $L^{\infty}$-bounds (11) for $n$ and $p$. We only detail the bound for $p$ and sketch how the bound for $n$ follows in a similar fashion. After recalling (with $\tau_{p}=1$ w.l.o.g.) 


$$
\partial_{t} p=\nabla \cdot J_{p}+\left(1-n_{t r}-\frac{p}{p_{0} e^{-V_{p}}} n_{t r}\right), \quad J_{p}=e^{-V_{p}} \nabla\left(p e^{V_{p}}\right),
$$

we introduce the variable $w=p e^{V_{p}}$ and observe that $\nabla \cdot J_{p}=\nabla \cdot\left(e^{-V_{p}} \nabla w\right)=e^{-V_{p}}\left(\Delta w-\nabla V_{p} \cdot \nabla w\right)$ and thus,

$$
\partial_{t} w=\Delta w-\nabla V_{p} \cdot \nabla w+e^{V_{p}}\left(1-n_{t r}-\frac{n_{t r}}{p_{0}} w\right)
$$

while the no-flux boundary condition $\hat{n} \cdot J_{p}=0$ on $\partial \Omega$ transforms to the homogeneous Neumann condition $\hat{n} \cdot \nabla w=0$ on $\partial \Omega$.

Next, by testing (85) with the positive part $(w-r-s t)_{+}:=\max \{0, w-r-s t\}$ for two constant $r, s>0$ to be chosen, we calculate by integration by parts in the first two terms

$$
\begin{aligned}
& \frac{d}{d t} \frac{1}{2} \int_{\Omega}(w-r-s t)_{+}^{2} d x \\
&=\int_{\Omega}(w-r-s t)_{+}\left(\Delta w-\nabla V_{p} \cdot \nabla w+e^{V_{p}}\left(1-n_{t r}-\frac{n_{t r}}{p_{0}} w\right)-s\right) d x \\
&=-\int_{\Omega} \mathbb{1}_{w \geq r+s t}|\nabla w|^{2} d x-\int_{\Omega} \nabla V_{p} \cdot \nabla \frac{(w-r-s t)_{+}^{2}}{2} d x \\
& \quad+\int_{\Omega}(w-r-s t)_{+}\left(e^{V_{p}}\left(1-n_{t r}-\frac{n_{t r}}{p_{0}} w\right)-s\right) d x \\
& \leq \frac{\left\|\Delta V_{p}\right\|_{L^{\infty}(\Omega)}}{2} \int_{\Omega}(w-r-s t)_{+}^{2} d x \\
&+\int_{\Omega}(w-r-s t)_{+}\left(e^{V_{p}}\left(1-n_{t r}-\frac{n_{t r}}{p_{0}} w\right)-s\right) d x
\end{aligned}
$$

since $\hat{n} \cdot V_{p} \geq 0$ by assumption (4). Moreover, since $n_{t r} \in[0,1]$ and $w \geq 0$, we have

$$
\begin{aligned}
\frac{d}{d t} \frac{1}{2} \int_{\Omega}(w-r-s t)_{+}^{2} d x \leq & \frac{\left\|\Delta V_{p}\right\|_{L^{\infty}(\Omega)}}{2} \int_{\Omega}(w-r-s t)_{+}^{2} d x \\
& +\int_{\Omega}(w-r-s t)_{+}\left(\left\|e^{V_{p}}\right\|_{L^{\infty}(\Omega)}-s\right) d x .
\end{aligned}
$$

Thus, by choosing $s:=\left\|e^{V_{p}}\right\|_{L^{\infty}(\Omega)}$ and $r:=\|w(\tau, \cdot)\|_{L^{\infty}(\Omega)}$ for some time $\tau \geq 0$, we conclude that

$$
\frac{d}{d t} \int_{\Omega}(w-r-s t)_{+}^{2} d x \leq\left\|\Delta V_{p}\right\|_{L^{\infty}(\Omega)} \int_{\Omega}(w-r-s t)_{+}^{2} d x,
$$

and a Gronwall lemma implies

$$
\|w(t, \cdot)\|_{L^{\infty}(\Omega)} \leq\|w(\tau, \cdot)\|_{L^{\infty}(\Omega)}+\left\|e^{V_{p}}\right\|_{L^{\infty}(\Omega)} t, \quad \text { for all } t \geq \tau \geq 0 .
$$

Transforming back, this yields 


$$
\begin{aligned}
\|p(t, \cdot)\|_{L^{\infty}(\Omega)} \leq & \frac{1}{\inf \left\{e^{V_{p}}\right\}}\left(\|p(\tau, \cdot)\|_{L^{\infty}(\Omega)}\left\|e^{V_{p}}\right\|_{L^{\infty}(\Omega)}+\left\|e^{V_{p}}\right\|_{L^{\infty}(\Omega)} t\right), \\
& \text { for all } t \geq \tau \geq 0 .
\end{aligned}
$$

In order to deduce the analogue bound for $n$ in (11), we consider (with $\tau_{n}=1$ w.l.o.g.)

$$
\partial_{t} n=\nabla \cdot J_{n}+\left(n_{t r}-\frac{n}{n_{0} e^{-V_{n}}}\left(1-n_{t r}\right)\right), \quad J_{n}=e^{-V_{n}} \nabla\left(n e^{V_{n}}\right) .
$$

We introduce the variable $\omega=n e^{V_{n}}$ and obtain in the same way as in (54)

$$
\partial_{t} \omega=\Delta \omega-\nabla V_{n} \cdot \nabla \omega+e^{V_{n}}\left(n_{t r}-\frac{1-n_{t r}}{n_{0}} \omega\right) .
$$

Following the same arguments as above,

$$
\|\omega(t, \cdot)\|_{L^{\infty}(\Omega)} \leq\|\omega(\tau, \cdot)\|_{L^{\infty}(\Omega)}+\left\|e^{V_{n}}\right\|_{L^{\infty}(\Omega)} t, \quad \text { for all } t \geq \tau \geq 0 .
$$

Transforming back, this yields

$$
\|n(t, \cdot)\|_{L^{\infty}(\Omega)} \leq \frac{1}{\inf \left\{e^{\left.V_{n}\right\}}\right.}\left(\|n(\tau, \cdot)\|_{L^{\infty}(\Omega)}\left\|e^{V_{n}}\right\|_{L^{\infty}(\Omega)}+\left\|e^{V_{n}}\right\|_{L^{\infty}(\Omega)} t\right), \text { for all } t \geq \tau \geq 0
$$

and thus (11).

Step 7: Regularity and bounds for $n_{t r}$. We still have to verify $n_{t r} \in C\left([0, T], L^{\infty}(\Omega)\right)$ for all $T>0$. Now, let $T>0$ and recall that

$$
n_{t r}(t)=n_{t r}(0)+\frac{1}{\varepsilon} \int_{0}^{t}\left(1-n_{t r}-e^{V_{p}} p n_{t r}-n_{t r}+e^{V_{n}} n\left(1-n_{t r}\right)\right) d s
$$

in $L^{2}(\Omega)$ for all $t \in[0, T]$. Considering a sequence $\left(t_{k}\right)_{k \in \mathbb{N}} \subset[0, T], t_{k} \rightarrow t$, we thus arrive at

$$
\begin{aligned}
\left\|n_{t r}\left(t_{k}\right)-n_{t r}(t)\right\|_{L^{\infty}(\Omega)} \leq & \frac{1}{\epsilon} \mid \int_{t}^{t_{k}} \| 1-n_{t r}-e^{V_{p}} p n_{t r}-n_{t r} \\
& +e^{V_{n}} n\left(1-n_{t r}\right) \|_{L^{\infty}(\Omega)} d s \mid \leq \frac{\left|t_{k}-t\right|}{\epsilon} C_{T} \rightarrow 0
\end{aligned}
$$

for $k \rightarrow \infty$. This proves the assertion.

The claim $\partial_{t} n_{t r} \in C\left([0, T], L^{2}(\Omega)\right)$ for all $T>0$ is an immediate consequence of the last equation in (83) together with the $L^{2}$-continuity and $L^{\infty}$-bounds of $u, v$ and $n_{t r}$.

Next, concerning the bounds (12), we recall system (1) and observe that for all $\varepsilon \in\left(0, \varepsilon_{0}\right]$

$$
\varepsilon \partial_{t} n_{t r}=h\left(n_{t r}\right):=R_{p}\left(p, n_{t r}\right)-R_{n}\left(n, n_{t r}\right)
$$


in the sense of $L^{2}(\Omega)$, where $h\left(n_{t r}=0\right) \geq \frac{1}{\tau_{p}}>0$ and $h\left(n_{t r}=1\right) \leq-\frac{1}{\tau_{n}}<0$ uniformly for all non-negative $n$ and $p$. Therefore, wherever $n_{t r, I}(x)=0$ (or analogously $n_{t r, I}(x)=1$ ), an elementary argument proves that $n_{t r}(t, x)$ grows (or decreases) linearly in time and decays back to 0 (or 1$)$ at most like $(a+b t)^{-1}$. More precisely, we reuse the transformed variable $w=p e^{V_{p}}$ and find

$$
\varepsilon \partial_{t} n_{t r} \geq \frac{1}{\tau_{p}}\left[1-\left(1+\frac{\tau_{p}}{\tau_{n}}+\frac{\|w\|_{L^{\infty}(\Omega)}}{p_{0}}\right) n_{t r}\right] \geq \frac{1}{\tau_{p}}\left[1-\left(1+\frac{\tau_{p}}{\tau_{n}}+\frac{r+s t}{p_{0}}\right) n_{t r}\right]
$$

for some constants $r$ and $s$ due to the estimate (86). Setting $\tau_{n}:=\tau_{p}:=1$ w.l.o.g., we have

$$
\varepsilon \partial_{t} n_{t r} \geq 1-(\widetilde{r}+\tilde{s} t) n_{t r}
$$

with appropriate $\tilde{r}, \tilde{s}>0$ independent of $\varepsilon$. By observing that the ODE $\varepsilon_{0} \dot{y}=1-(\widetilde{r}+\tilde{s} t) y$ features the positive nullcline $y_{0}(t)=1 /(\widetilde{r}+\widetilde{s} t)$, which moreover attracts all solution trajectories, standard comparison arguments (pointwise in $x \in \Omega$ ) imply that for all times $\tau>0$, there exist positive constants $\eta=\eta\left(\varepsilon_{0}, \tau, \tau_{n}, \tau_{p}\right)$, $\theta=\theta\left(C_{n}, C_{p}, K_{n}, K_{p}\right)$ and a sufficiently small constant $\gamma\left(\tau, C_{n}, C_{p}, K_{n}, K_{p}\right)>0$ such that

$$
n_{t r}(t, x) \geq \min \left\{\eta t, \frac{\gamma}{1+\theta t}\right\} \quad \text { for all } t \geq 0 \text { and a.e. } x \in \Omega
$$

where $\eta \tau=\frac{\gamma}{1+\theta \tau}$ such that the linear and the inverse linear bound intersect at time $\tau$.

Finally, the upper bounds (12) follow from analogue arguments.

Step 8: Lower bounds for $n$ and p. Finally, we prove the bounds (13). We will only detail the argument for the lower bound on $n$, as the bound for $p$ follows in an anlogue way. Recalling the transformed equation for $\omega=e^{V_{n}} n$ (satisfying $\hat{n} \cdot \nabla \omega=0$ on $\partial \Omega$ ), we estimate

$$
\partial_{t} \omega=\Delta \omega-\nabla V_{n} \cdot \nabla \omega+e^{V_{n}}\left(n_{t r}-\frac{1-n_{t r}}{n_{0}} \omega\right) \geq \Delta \omega-\nabla V_{n} \cdot \nabla \omega-\alpha \omega+c n_{t r}
$$

where $\alpha>0$ and $c>0$ are positive constants due to the assumptions (4) and $e^{V_{n}} \omega n_{t r} \geq 0$.

Next, we use (12), i.e. that for all $\tau>0$ fixed, there exist constants $\eta, \theta$ and $\gamma$ such that $n_{t r}(t, x) \geq \eta t$ for all $0 \leq t \leq \tau$ and a.e. $x \in \Omega$, while $n_{t r}(t, x) \geq \gamma /(1+\theta t)$ for all $t \geq \tau$ and a.e. $x \in \Omega$. Then, by introducing the negative part $(\omega)_{-}:=\min \{\omega, 0\}$ and testing (90) with $\left(\omega-\frac{\mu t^{2}}{2}\right)_{-}$for a constant $\mu>0$ to be chosen below, we estimate 


$$
\begin{aligned}
\frac{d}{d t} & \frac{1}{2} \int_{\Omega}\left(\omega-\frac{\mu t^{2}}{2}\right)_{-}^{2} d x=\int_{\Omega}\left(\omega-\frac{\mu t^{2}}{2}\right)_{-}\left(\partial_{t} \omega-\mu t\right) d x \\
= & \int_{\Omega}\left|\left(\omega-\frac{\mu t^{2}}{2}\right)_{-}\right|\left(-\partial_{t} \omega+\mu t\right) d x \\
\leq & \int_{\Omega}\left|\left(\omega-\frac{\mu t^{2}}{2}\right)_{-}\right|\left(-\Delta \omega+\nabla V_{n} \cdot \nabla \omega+\alpha \omega-c n_{t r}+\mu t\right) d x \\
= & \int_{\Omega}\left(\omega-\frac{\mu t^{2}}{2}\right)_{-}\left(\Delta \omega-\nabla V_{n} \cdot \nabla \omega\right) d x+\int_{\Omega}\left|\left(\omega-\frac{\mu t^{2}}{2}\right)_{-}\right|\left(\alpha \omega-c n_{t r}+\mu t\right) d x \\
\leq & -\int_{\Omega} \mathbb{1} \omega \leq \frac{\mu t^{2}}{2}|\nabla \omega|^{2} d x-\frac{1}{2} \int_{\Omega} \nabla\left(\omega-\frac{\mu t^{2}}{2}\right)_{-}^{2} \cdot \nabla V_{n} d x \\
& +\int_{\Omega}\left|\left(\omega-\frac{\mu t^{2}}{2}\right)_{-}\right|\left(\alpha \omega-c n_{t r}+\mu t\right) d x .
\end{aligned}
$$

Thus, for $0 \leq t \leq \tau$ when $n_{t r}(t, x) \geq \eta t$, we have

$$
\begin{aligned}
\frac{d}{d t} \frac{1}{2} \int_{\Omega}\left(\omega-\frac{\mu t^{2}}{2}\right)_{-}^{2} d x \leq & \int_{\Omega}\left(\omega-\frac{\mu t^{2}}{2}\right)_{-}^{2} \frac{\Delta V_{n}}{2} d x \\
& +\int_{\Omega}\left|\left(\omega-\frac{\mu t^{2}}{2}\right)_{-}\right|\left(\alpha \frac{\mu t^{2}}{2}-c \eta t+\mu t\right) d x
\end{aligned}
$$

If we choose $\mu\left(\alpha \frac{\tau}{2}+1\right) \leq c \eta$, we obtain

$$
\frac{d}{d t} \int_{\Omega}\left(\omega-\frac{\mu t^{2}}{2}\right)_{-}^{2} d x \leq\left\|\Delta V_{n}\right\|_{L^{\infty}(\Omega)} \int_{\Omega}\left(\omega-\frac{\mu t^{2}}{2}\right)_{-}^{2} d x .
$$

Hence, since $\int_{\Omega}(\omega(0, x))_{-}^{2} d x=0$, we deduce from a Gronwall lemma

$$
\int_{\Omega}\left(\omega-\frac{\mu t^{2}}{2}\right)_{-}^{2} d x=0, \quad \text { for all } 0 \leq t \leq \tau,
$$

which yields in particular $\omega(t, x) \geq \frac{\mu t^{2}}{2}$ for all $0 \leq t \leq \tau$ and a.e. $x \in \Omega$.

Moreover, for $t \geq \tau$ when $n_{t r}(t, x)^{2} \geq \frac{\gamma}{1+\theta t}$, we test (90) with $\left(\omega-\frac{\Gamma}{1+\theta t}\right)_{-}$for a constant $\Gamma>0$ to be chosen below, and estimate similar to above

$$
\begin{aligned}
\frac{d}{d t} \frac{1}{2} & \int_{\Omega}\left(\omega-\frac{\Gamma}{1+\theta t}\right)_{-}^{2} d x=\int_{\Omega}\left(\omega-\frac{\Gamma}{1+\theta t}\right)_{-}\left(\partial_{t} \omega+\frac{\Gamma \theta}{(1+\theta t)^{2}}\right) d x \\
\leq & \int_{\Omega}\left(\omega-\frac{\Gamma}{1+\theta t}\right)_{-}\left(\Delta \omega-\nabla V_{n} \cdot \nabla \omega-\alpha \omega+c n_{t r}+\frac{\Gamma \theta}{(1+\theta t)^{2}}\right) d x \\
\leq & -\int_{\Omega} \mathbb{1}_{\omega \leq \frac{\Gamma}{1+\theta t}}|\nabla \omega|^{2} d x-\frac{1}{2} \int_{\Omega} \nabla\left(\omega-\frac{\Gamma}{1+\theta t}\right)_{-}^{2} \cdot \nabla V_{n} d x \\
& +\int_{\Omega}\left|\left(\omega-\frac{\Gamma}{1+\theta t}\right)_{-}\right|\left(\alpha \omega-c n_{t r}-\frac{\Gamma \theta}{(1+\theta t)^{2}}\right) d x .
\end{aligned}
$$


And as $n_{t r}(t, x) \geq \frac{\gamma}{1+\theta t}$ for $t \geq \tau$, we find

$$
\begin{aligned}
\frac{d}{d t} & \frac{1}{2} \int_{\Omega}\left(\omega-\frac{\Gamma}{1+\theta t}\right)_{-}^{2} d x \leq \int_{\Omega}\left(\omega-\frac{\Gamma}{1+\theta t}\right)_{-}^{2} \frac{\Delta V_{n}}{2} d x \\
& +\int_{\Omega}\left|\left(\omega-\frac{\Gamma}{1+\theta t}\right)_{-}\right|\left(\alpha \frac{\Gamma}{1+\theta t}-c \frac{\gamma}{1+\theta t}-\frac{\Gamma \theta}{(1+\theta t)^{2}}\right) d x .
\end{aligned}
$$

Choosing $\alpha \Gamma \leq c \gamma$, we arrive at

$$
\frac{d}{d t} \int_{\Omega}\left(\omega-\frac{\Gamma}{1+\theta t}\right)_{-}^{2} d x \leq\left\|\Delta V_{n}\right\|_{L^{\infty}(\Omega)} \int_{\Omega}\left(\omega-\frac{\Gamma}{1+\theta t}\right)_{-}^{2} d x .
$$

By further reducing either $\Gamma$ or $\mu$, we are able to satisfy $\frac{\Gamma}{1+\theta \tau}=\frac{\mu \tau^{2}}{2}$. On the one hand, this implies that $\int_{\Omega}\left(\omega(\tau, x)-\frac{\Gamma}{1+\theta \tau}\right)_{-}^{2} d x=0$, which results-by using a Gronwall argument-in

$$
\int_{\Omega}\left(\omega(t, x)-\frac{\Gamma}{1+\theta t}\right)_{-}^{2} d x=0, \quad \text { for all } t \geq \tau,
$$

and, hence, $\omega(t, x) \geq \frac{\Gamma}{1+\theta t}$ for all $t \geq \tau$ and a.e. $x \in \Omega$. On the other hand, the increasing and decreasing bounds now again intersect at time $\tau$ as desired.

\section{References}

1. Alikakos, N.D.: $\$\{\mathrm{~L}\}^{\wedge} \mathrm{p} \$$ bounds of solutions of reaction-diffusion equations. Commun. Partial Differ. Equ. 4(8), 827-868 (1979)

2. Beesak, P.R.: Gronwall Inequalities, vol. 11, Carleton Math. Lecture Notes (1975)

3. Chipot, M.: Elements of Nonlinear Analysis. Birkhäuser Advanced Texts. Birkhäuser, Basel (2000)

4. Desvillettes, L., Fellner, K.: Entropy methods for reaction-diffusion equations: degenerate diffusion. DCDS Supplements Special, pp. 304-312 (2007)

5. Desvillettes, L., Fellner, K.: Entropy methods for reaction-diffusion equations: slowly growing a priori bounds. Rev. Mat. Iberoam. 24, 407-431 (2008)

6. Desvillettes, L., Fellner, K.: Exponential convergence to equilibrium for a nonlinear reaction-diffusion system arising in reversible chemistry. Syst. Model. Optim. IFIP AICT 443, 96-104 (2014)

7. Di Francesco, M., Fellner, K., Markowich, P.: The entropy dissipation method for inhomogeneous reaction-diffusion systems. Proc. R. Soc. A 464, 3272-3300 (2008)

8. Einav, A., Morgan, J., Tang, B.Q.: Indirect diffusion effect in degenerate reaction-diffusion systems. arxiv:2001.00852v1

9. Fellner, K., Kniely, M.: On the entropy method and exponential convergence to equilibrium for a recombination-drift-diffusion system with self-consistent potential. Appl. Math. Lett. 79, 196-204 (2018)

10. Fellner, K., Tang, B.Q.: Explicit exponential convergence to equilibrium for nonlinear reaction-diffusion systems with detailed balance condition. Nonlinear Anal. 159, 145-180 (2017)

11. Fellner, K., Rosenberger, S., Tang, B.Q.: Quasi-steady-state approximation and numerical simulation for a volume-surface reaction-diffusion system. Commun. Math. Sci. 14, 1553-1580 (2016)

12. Fellner, K., Prager, W., Tang, B.Q.: The entropy method for reaction-diffusion systems without detailed balance: first order chemical reaction networks. Kinet. Relat. Mod. 10, 1055-1087 (2017)

13. Fellner, K., Latos, E., Tang, B.Q.: Global regularity and convergence to equilibrium of reaction-diffusion systems with nonlinear diffusion. J. Evol. Equ. (2019) 
14. Gilbarg, D., Trudinger, N.S.: Elliptic Partial Differential Equations of Second Order. Springer, Berlin (1977)

15. Goudon, T., Miljanović, V., Schmeiser, C.: On the Shockley-Read-Hall model: generation-recombination in semiconductors. SIAM J. Appl. Math. 67, 1183-1201 (2007)

16. Hall, R.N.: Electron-hole recombination in Germanium. Phys. Rev. 87, 387 (1952)

17. Hsieh, C.-Y., Lin, T.-C.: Exponential decay estimates for the stability of boundary layer solutions to Poisson-Nernst-Planck systems: one spatial dimension case. SIAM J. Math. Anal. 47, 3442-3465 (2015)

18. Markowich, P.A., Ringhofer, C.A., Schmeiser, C.: Semiconductor Equations. Springer, Wien (1990)

19. Mielke, A., Haskovec, J., Markowich, P.A.: On uniform decay of the entropy for reaction-diffusion systems. J. Dyn. Differ. Equ. 27, 897-928 (2015)

20. Mock, M.S.: Analysis of Mathematical Models of Semiconductor Devices. Advances in Numerical Computation Series, vol. 3. Boole Press, Dublin (1983)

21. Shockley, W., Read, W.T.: Statistics of the recombinations of holes and electrons. Phys. Rev. 87, 835-842 (1952)

22. Stroock, D.: Logarithmic Sobolev inequalities for Gibbs states. Lect. Notes Math. 1563, 194-228 (1993)

23. Taylor, M.E.: Partial Differential Equation III-Nonlinear Equations. Springer Series Applied Mathematical Sciences, vol. 117. Springer, New York (1996)

24. Van Roosbroeck, W.: Theory of the flow of electrons and holes in Germanium and other semiconductors. Bell Syst. Tech. J. 29, 560-607 (1950)

25. Willett, D.: A linear generalization of Gronwall's inequality. Proc. Am. Math. Soc. 16, 774-778 (1965)

26. Wu, H., Markowich, P.A., Zheng, S.: Global existence and asymptotic behavior for a semiconductor drift-diffusion-Poisson model. Math. Models Methods Appl. Sci. 18, 443-487 (2008)

Publisher's Note Springer Nature remains neutral with regard to jurisdictional claims in published maps and institutional affiliations. 